LBL- -33379

DE93 007701

\title{
DX Centers in III-V Semiconductors under Hydrostatic Pressure
}

\author{
Jeffrey Alan Wolk \\ Ph.D. Thesis \\ Department of Physics \\ University of California \\ and \\ Center for Advanced Materials \\ Materials Sciences Division \\ Lawrence Berkeley Laboratory \\ University of California \\ Berkeley, CA 94720
}

November 1992

This work was supported by the Director, Office of Energy Research, Office of Basic Energy Sciences, Materials Sciences Division, of the U.S. Departinent of Energy under Contract No. DE-AC03-76SF00098. 


\title{
DX Centers in III-V Semiconductors under Hydrostatic Pressure
}

\author{
Copyright $\odot 1992$
}

by

Jeffrey Alan Wolk

The U.S. Department of Energy has the right to use this document for any purpose whatsoever including the right to reproduce all or any part thereof 


\author{
Abstract \\ DX Centers in III-V Semiconductors \\ Under Hydrostatic Pressure \\ by \\ Jeffrey Alan Wolk \\ Doctor of Philosophy in Physics \\ University of California at Berkeley \\ Professor Eugene E. Haller, Co-Chairman \\ Professor Leopoldo M. Falicov, Co-Chairman
}

DX centers are deep level defects found in some III-V semiconductors and their alloys. These defects are characterized by several unusual physical properties, including persistent photoconductivity and a large difference between their thermal and optical ionization energies. We have used hydrostatic pressure as a tool to study the microscopic structure of these defects and examine whether their existence is a general feature of all III-V semiconductors.

We have observed a new local vibrational mode (LVM) in hydrostatically stressed, Si-doped GaAs. The corresponding infrared absorption peak is distinct from the Siga shallow donor LVM peak, which is the only other LVM peak observed in our samples, and is assigned to the Si DX center. Analysis of the relative intensities of the Si DX LVM and the Si shallow donor LVM peaks has been combined with Hall effect and resistivity analysis to infer that the $\mathrm{Si}$ DX center is negatively charged. The frequency of this new mode provides important clues to the structure of this defect.

We have also discovered a pressure induced deep donor level in Sdoped InP which has the properties of a DX center. The pressure at which the 
new defect becomes more stable than the shallow donor is $82 \mathrm{kbar}$. We have measured the optical ionization energy anc' the energy dependence of the optical absorption cross section for this new defect. We have also determined the capture barrier from the conduction band into the DX state. The fact that DX centers can be formed in InP by applying pressure suggests that the existence of DX states should be very common in n-type III-V semiconductors. We also suggest a method for predicting under what conditions these defects will be the most stable form of the donor impurity. 


\section{Table of Contents}

Acknowledgments vi

Chapter 1. Introduction to DX Centers 1

1.1. Introduction to Deep Levels in Semiconductors..............................................

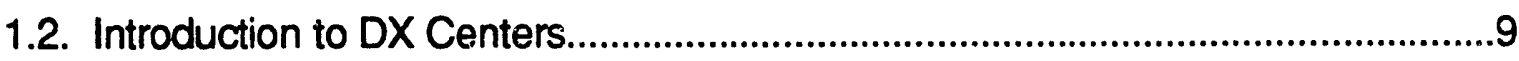

Chapter 2. High Pressure Experimental Work Using Diamond Anvil Cells....16

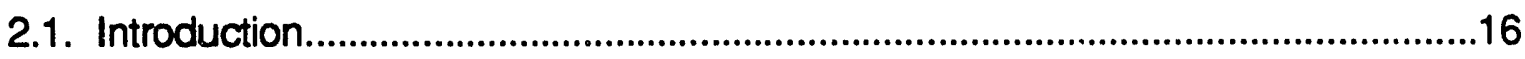

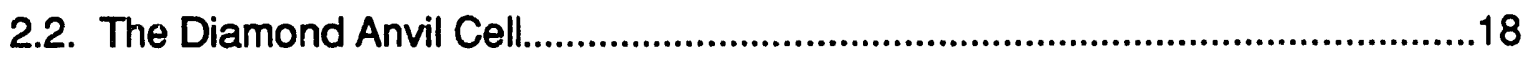

2.2.1. Introduction to the Diamond Anvil Cell.............................................18

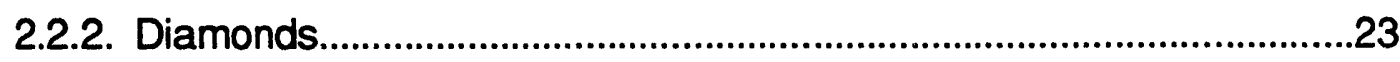

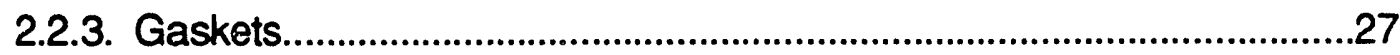

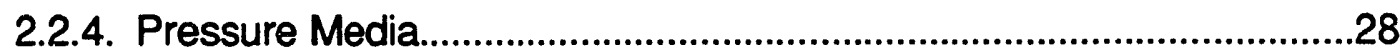

2.2.5. Loading the Diamond Anvil Cell.........................................................30

2.2.6. Sample Preparation..................................................................................34

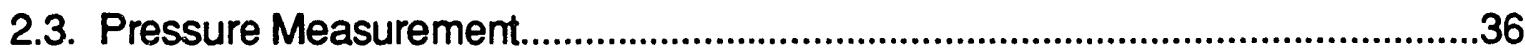

2.3.1. Ruby Fluorescence Scale....................................................................36

2.3.2. Pressure Measurement System.............................................................38

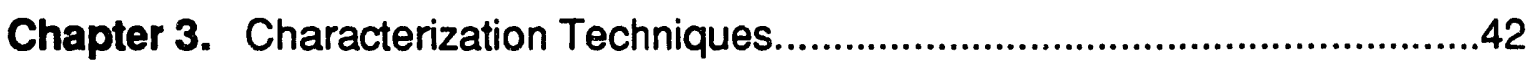

3.1. Fourier Transform and Grating Spectroscopy.............................................42

3.1.1. Fourier Transform Spectroscopy.........................................................42

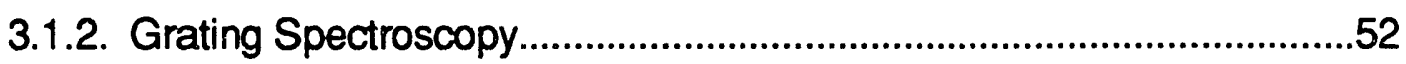


3.1.3. Comparison of Fourier Transform and Grating Spectroscopy.......57

3.1.4. Optica! Spectroscopy in the Diamond Anvil Cell..............................59

3.2 Hall Effect. .63

Chapter 4. Local Vibrational Mode Spectruscopy of DX Centers in GaAs:Si.

4.1. Introduction to Local Vibrational Modes and their Observation .69

4.1.1. Theoretical Description of Local Vibrational Modes .69

4.1.2 Observation of Local Vibrational Modes .79

4.2. Motivation for the Experiment. .82

4.2.1. Microscopic Models for the DX Center .82

4.2.1.1. The Chadi anci Chang Model. .82

4.2.1.2. The $A_{1}$ state .93

4.2.2. Summary of Previous Experimental Work on DX Centers..

4.3. Local Vibrational Mode Spectroscopy of DX Centers in GaAs Under Hydrostatic Pressure. 100

4.4. Comparison Between Experiment and Theory.

Chapter 5. Discovery of DX Center in InP:S. .122

5.1. Motivation for the Experiment. 122

5.2. Determination of the DX Center Transition Pressure .125

5.2.1 The Restrahlen Band 125

5.2.2 Determining the Shallow-to-Deep Transition Pressure. 132

5.3. Determining the Optical lonization Energy, $E_{o p t}$, of the InP:S DX Center. 
5.3.1. Theoretical Background of Optical lonization of Deep Levels in Semiconductors.

5.3.2. Experimental Determination of the DX Center Optical Ionization Energy.

5.4. Determining the Thermal Capture Barrier from the Shallow

Donor State into the DX State. 154

5.4.1. Modelling the Cjapture of Free Carriers by DX Centers. 154

5.4.2. Experimenta! Determination of the Capture Barrier from the Shallow State into the DX state. 160

Chapter 6. Conclusion. 167

References. .170 


\section{Acknowledgments}

The writing of this thesis is a testament not only to the work I have performed during my tenure as a graduate student, but also to the large number of people who have generously shared their time and considerable talents in helping me to achieve this goal.

I owe the greatest debt of gratitude to my advisors Eugene Haller and Leo Falicov, whose advice and guidance have been invaluable in my transition from a beginning graduate student into a physicist. I am grateful for their help when it was necessary, and for their granting me the independence to discover and learn for myself.

The staff of Lawrence Berkeley Laboratory have also been of tremendous help to me during my work here. I am especially indebted to Jeff Beeman, who gave suggestions and assistance in more aspects of my research than he would probably care to recall, and who also constructed the photoconductor detectors which made this research possible. I am grateful to Wladek Walukiewicz, whose office door was always open when I needed help in understanding some theoretical aspect of my research, and whose theoretical work was invaluable in the interpretation of my data. I would like to express my thanks to Bill Hansen, who always had ideas on how to tackle a problem when it seemed I had run out, and Dick Davis, who kept me supplied with whatever I required. I would like to express my gratitude to Professor Raymond Jeanloz, who shared his expertise on high pressure work and allowed me to use his high pressure laboratory throughout my work. I am also grateful to him and Professor Peter Yu for reading this manuscript. I would like to thank Edith Bourret-Courchesne and Joseph Guitron for their help in obtaining, growing, and processing a wide variety of GaAs samples. I am also 
grateful to Margaret Ragsdale for her efficiency and care in accomplishing all of the administrative tasks I asked of her, and her constant willingness to handle urgent requests.

My fellow graduate students, who in addition to having made my graduate experience fun, have also made invaluable contributions towards my research. I would like to thank in particular Michael Kruger, who taught me a large part of what I know about high pressure work and made crucial contributions to the development of the high pressure optical spectroscopy system developed as part of my research. I would also like to give special thanks to James Heyman for his help with many aspects of my work and his critical discussions of my research. I have also enjoyed collaborating in various ways with the other students in our group, namely Kohei Itoh, Amy Moll, Oscar Dubon, Jr., David Bliss, and In Chin Wu, many of whom formed the core of the best (though certainly not in the won-lost record sense) softball team I have ever had the pleasure to play on.

I would finally like to give thanks to my parents, Ron and Eileen, who have always supported whatever path I have chosen in my life, and given me the faith in myself to accomplish whatever task is set before me. I would also like to thank my brothers Brad and Steve for providing support and understanding when I needed it. Finally, I thank my wife, RuthAnne, whose love, support, patience, and pride in my accomplishments have made my time at Berkeley such a fulfilling part of my life. 


\section{Introduction to DX Centers}

\subsection{Introduction to Deep Levels in Semiconductors}

Semiconducting materials are characterized by a fundamental band gap $E_{g}$ in the range of approximately 0-3 electron volts. Because of this, their electrical conductivity is intermediate between that of a metal $\left(E_{g}=0\right)$ and that of an insulator $(E>3 \mathrm{eV})$. The usefulness of semiconductors lies largely in the ability to manipulate, in a controlled manner, their electrical conductivity over more than ten orders of magnitude. This manipulation is normally achieved through the introduction of impurity atoms into the seiticonductor, making possible the creation of semiconducting materials with behavior ranging from nearly metallic to nearly insulating. Impurities are usually categorized as either shallow and deep. Shallow impurities introduce electronic energy levels slightly below the bottom of the conduction band (donors) or slightly above the top of the valence band (acceptors). The carriers in these levels are easily excited to the nearby bands and thus contribute to the conductivity of the crystal. Deep impurities create electronic levels which are further removed from the band edges than shallow levels, although it will be shown later that it is not necessarily a straightforward matter to characterize a level as deep based solely on this criterion.

The essential physics of shallow impurities in semiconductors has been understood within the framework of effective mass theory for over thirty years (Kohn, 1957). This theory treats the potential of the impurity as a perturbation of the host lattice potential. The basic ideas of this formalism can be understood by considering a $\mathrm{P}$ impurity replacing a $\mathrm{Si}$ atom in a $\mathrm{Si}$ lattice. The $\mathrm{P}$ atom has five valence electrons, one more than the surrounding $\mathrm{Si}$ atoms. Only four of the five 
of these electrons are occupied in bonding to the Si lattice, so the defect can be thought of as an electron and a positively charged $P$ atom. If the extra electron is not bound to the $P$ atom and is moving through the crystal, then it travels with energy $E_{C B M}+(\hbar k)^{2} / 2 m^{*}$, where $E_{C B M}$ is the energy of the conduction band minimum, $k$ is the electron momentum, and $m^{*}$ is the effective mass of an electron associated with that band. The electron can, however, become trapped by the Coulomb field of the $P$ atom. The donor atom along with the trapped electron forms a defect system which is analogous to a hydrogen atom. It is therefore not surprising that a detailed calculation shows that the ground state energy level, $E_{d}$, and radius, $r_{d}$, of the donor impurity are given by slight modifications of the formulas appropriate for an isolated $\mathrm{H}$ atom. We have

$$
E_{d}=E_{C B M}-\frac{e^{4} m^{*}}{2\left(4 \pi \varepsilon \varepsilon_{0} \hbar\right)^{2}}
$$

and

$$
r_{d}=\frac{4 \pi \varepsilon \varepsilon_{0} \hbar^{2}}{m^{*} e^{2}}
$$

where $e$ is the charge on the electron, $\varepsilon$ is the static dielectric constant of the material, and $\varepsilon_{0}$ is the permittivity of free space. The fact the the donor is embedded in a lattice is taken into account by 1) the static dielectric constant, which corrects for the fact that the coulomb attraction between the charges is reduced as a result of the polarization of the lattice by the positive nucleus, and 2) the effective mass of the electron, which accounts for the fact that the motion of the electron is affected by the potential of the lattice. Using the values 
appropriate for Si gives a binding energy of roughly $30 \mathrm{meV}$ and a Bohr radius of approximately $30 \AA$.

This large orbit justifies the use of the dielectric constant of the material since it will average the effect of the lattice over many lattice constants. It also justifies the use of the effective mass of the electron. If the wavefunction is very spread out in real space, then it is easy to show through Fourier analysis that it is very localized in momentum or $k$ space. The wavefunction of the impurity electron is thus comprised solely of band wavefunctions very close to the conduction band minimum. Since $\left(1 / \mathrm{m}^{*}\right)=\left(1 / \hbar^{2}\right)\left(\mathrm{d}^{2} \mathrm{E}_{\text {cond. band }} / \mathrm{dk}^{2}\right)$, this implies that the mass of the electron is determined by the curvature of the portion of the conduction band very close to this mil:m.

It is clear that this formulation will break down if the impurity has a strong short range potential, resulting in a much more localized wavefunction. The polarization of the lattice then cannot be taken into account through the static dielectric constant. It will also no longer be possible to associate the impurity level with a single conduction band minimum since the level will be spread out in momentum space. Levels with a strong, short-range potential are referred to as deep levels because they often introduce levels in the forbidden gap well removed from both the conduction and valence bands. In order to treat deep levels theoretically, the impurity potential $V_{i m p}$ and lattice potential $V_{\text {lat }}$ must be treated with equal care. It is no longer reasonable to treat $V_{i m p}$ as a perturbation.

An examination of Eqs. 1.1 and 1.2 shows that for a shallow level, a smaller wavefunction radius leads to an electronic energy deeper within the gap. This line of thinking breaks down in the case of deep levels. If we expand the impurity wavefunction $\psi$ in terms of the eigenfunctions $\phi_{\mathrm{nk}}$ of the perfect lattice, then 


$$
\psi \cdot \sum A_{n, k} \phi_{n, k} .
$$

As opposed to the case for shallow levels, the coefficients $A_{n, k}$ are significant over a large range of the Brillouin zone. The electronic energy of the localized state is given by

$$
E=\langle\psi \mid H d \psi\rangle\rangle+\left\langle\psi\left|V_{\text {imp }}\right| \psi\right\rangle
$$

where $\mathrm{H}_{0}$ is the Hamiltonian of the unperturbed lattice. Considering just the first matrix element in Eq. 1.4, using the fact that $H_{0} \phi_{n, k}=E_{n, k} \phi_{n, k}$, we find

$$
\left\langle\psi\left|H_{0}\right| \psi\right\rangle=\sum A_{n, k} A_{n, k}^{*} E_{n, k}
$$

Because bandwidths are on the order of a few eV, even small coefficients $A_{n, k}$ far from the band edges, where $E_{n, k}$ is large, can play an important role in determining the energy of a deep level. The quantity $A_{n, k} A_{n, k}^{*}$ is always positive. Therefore, in the case that the final level lies in the forbidden gap, which is in the middle of the range of $E_{n, k}$, there must be both positive and negative contributions to the sum in Eq 1.5. It is therefore the delicate cancellation between many large terms which determines the final energy level of the defect. This makes clear that there is no simple correlation between the energy of a deep level and its degree of localization. For this reason it is more illuminating to refer to levels as localized or delocalized rather than shallow or deep. 
A further complication when considering deep levels is that the localization of the defect results in its being strongly coupled to the lattice. The total defect Hamiltonian can be written as

$$
H_{\text {total }}=H_{e}+H_{1}+H_{e l}
$$

Here $\mathrm{H}_{\mathrm{e}}$ represents the electronic states when the ions of the lattice are at their equilibrium positions and $H_{1}$ is a harmonic oscillator Hamiltonian with eigenfunctions $\eta(Q)$, where $Q$ describes the displacement of the lattict ans and $Q=0$ refers to an undistorted lattice. $Q$ is commonly referred to as a configuration coordinate. The term $\mathrm{H}_{\mathrm{el}}$ represents the electron-lattice interaction. The gap state is associated with an impurity potential $V(r, Q)$ and a one-electron wavefunction $\Psi(r, Q)$. The Schrödinger equation is:

$$
\left(H_{e}+H_{1}+H_{e l}\right) \psi(r, Q)=\varepsilon(Q) \psi(r, Q)
$$

where

$$
\Psi(r, Q)=\Psi(r, Q) \eta(Q)
$$

and

$$
H_{\theta} \Psi(r, 0)=E(0) \Psi(r, 0),
$$

where $E(0)$ is the electronic contribution to the total energy at $Q=0$. If $H_{e l}=0$, then the quantity $\varepsilon(Q)$ can be expanded about its minimum value to give 


$$
\varepsilon(Q)=\varepsilon(Q=0)+\frac{1}{2} \sum_{\alpha} k_{\alpha}\left(Q_{\alpha}\right)^{2},
$$

where $k_{\alpha}$ is a force constant and the sum is over different lattice modes.

We now consider the effect on Eq. 1.10 if $\mathrm{H}_{\mathrm{el}}$ is not equal to zero. We assume that we are cnly concerned with one lattice mode, described by $Q$, and that changes in the electronic wavefunction and potential $V$ with respect to $Q$ are small. Expanding $V(r, Q)$ in a Taylor series around $Q=0$ gives

$$
V(r, Q)=V(r, 0)+\left.Q(\partial V(r, Q) / \partial Q)\right|_{Q=0}+\cdots \cdot
$$

The electron-lattice interaction $\mathrm{H}_{\mathrm{el}}$ represents the change in the potential $\mathrm{V}$ as a function of the nuclear displacement. Therefore, retaining only terms linear in $Q$, $H_{e l}=\left.Q(\partial V / \partial Q)\right|_{Q}=0$. The total energy of the electronic state in the presence of lattice coupling can thus be written in the form

$$
\varepsilon(Q)=E(0)+\alpha Q+\frac{1}{2} \beta(Q)^{2} .
$$

This means that the position of the lattice will be different depending on whether or not the defect is occupied since this will change the value of $\alpha$. We also have implicitly used the Born-Oppenheimer approximation in this discussion, which states that the nuclei are assumed to move slowly in comparison to the electrons because of their much larger mass. This means that the electrons can always follow the lattice motion and that the latter will not induce any electronic transitions.

The total energy of the defect can be represented graphically in a configuration coordinate diagram, such as that shown in Figure 1.1. This type of 


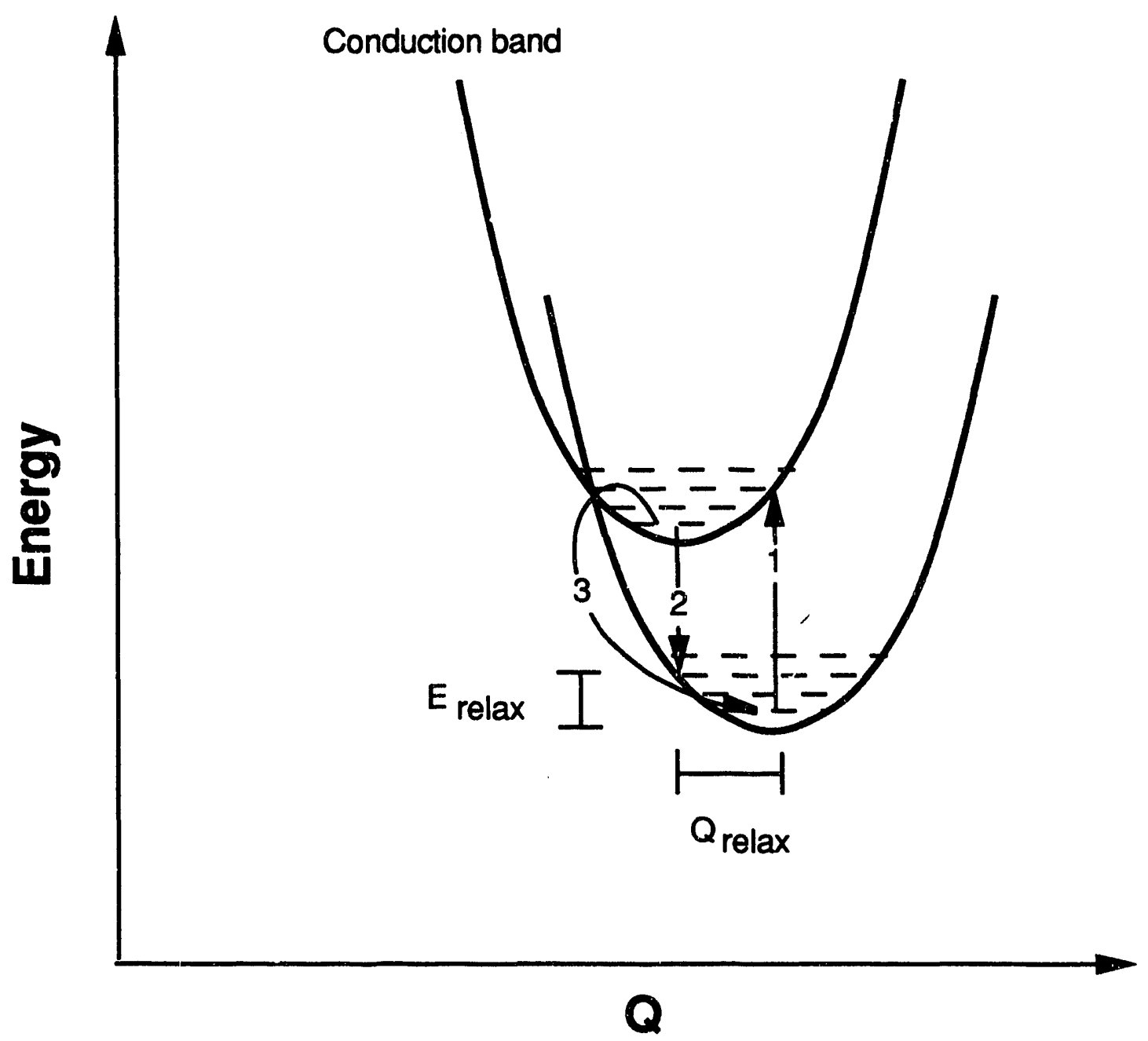

Fig 1.1. Configuration coordinate diagram for a defect showing optical and thermal transitions between two charge states of the defect. 
diagram is a plot of the energy $E$ of the defect (lattice plus electronic energy) versus the configuration coordinate Q. In accordance with Eq. 1.12, the levels are represented by parabolas. For simplicity, we assume that $\beta$ for the conduction band and defect states are equal. The horizontal dashed lines represent the vibrational eigenstates of the harmonic oscillator defect system. The arrows represent different transitions which may occur. As an example we take the lower curve as the occupied state of the defect. Upon carrier emission, the lattice will distort by the amount

$$
Q_{\text {relax }}=\left(\alpha_{\text {unocc }}-\alpha_{\text {occ }}\right) / k \text {, }
$$

with the corresponding relaxation energy

$$
E_{\text {rel }: x}=-(1 / 2)\left(\alpha_{\text {unocc }}-\alpha_{\text {occ }}\right)^{2 / k}
$$

Transition 1 represents optical ionization of the defect. The optical transitions are vertical on this diagram because they take place in a time which is much too short for the lattice to change its configuration. This is referred to as the FrankCondon principle (Condon, 1929). After ionization, the lattice relaxes to $Q=Q^{\prime}$ by the emission of phonons. The relaxation energy is Sh $\omega$, where $S$ is the Huang-Rhys (Huang, 1951) factor and $\omega$ is the frequency of the phonon emitted in the relaxation process. The Huang-Rhys factor is essentially the number of phonons emitted in the lattice relaxation, and can be taken as a measure of the strength of the electron-phonon coupling. Transition 2 is photoluminescence from the band back to the deep level. The difference in energy between transitions 1 and 2 is referred to as the Franck-Condon shift, and is given by $d_{F C}$ $=2 E_{\text {relax }}$. Transition 3 represents a thermal transition referred to as multi- 
phonon capture. The carrier is thermally excited over the barrier and emits phonons as it relaxes to the lowest energy state. These diagrams can be used to understand many of the properties of deep levels, and are used for exactly this purpose in the next section to understand the fascinating behavior of DX centers.

\subsection{Introduction to DX Centers}

Metastable defects are a special class of semiconductor impurities which are stable in two different lattice configurations. In some cases, one configuration introduces a shallow level into the gap while the other configuration creates a deep level. If the different lattice configurations correspond to different charge states then the defect is referred to as bistable. Bistable defects can obviously be transformed from one lattice configuration to another by either carrier emission or capture. Donor impurities in some III-V semiconductors are bistable, and the deep level configuration of these impurities is known as the DX center.

The DX center has been observed in $\left.A\right|_{x} G_{1-x} A s$ for $x \geq 0.22$ (Nelson, (1977); Lang (1977)) and GaAs under hydrostatic pressure (Mizuta, 1985) greater than approximately $20 \mathrm{kbar}$. It is characterized by several unusial physical properties, including a large difference between its thermal and optical ionization energies, extremely small capture cross sections, and persistent photoconductivity (Lang, 1977). In addition to these fascinating characteristics, the DX center is important from a technological standpoint since the electrical characteristics of a semiconducting material are affected by the relative stability of the shallow and deep form of the defect. For example, this issue is crucial in 
the design of semiconductor lasers where it is desirable to have heavily doped layers of $A l_{x} \mathrm{Ga}_{1-x} A s$ with $x>0.3$, above the alloying concentration at which the deep DX levels are the stable form of the donor. If the ration of the concentration of the two defects is $n_{\text {shallow donor }} / n_{D X}=0.01$, then in order to achieve the desired free carrier concentration one must put 100 times as many donor impurities as compared to when the deep form of the donor is not present. This results in problems with dopant diffusion when the device is heated during processing. These fundamental and technological issues have stimulated a great number of experimenters to study this defect since it was first identified in 1977.

The DX center was named based on the following two observations. First, it was observed that the concentration of this deep defect was proportional to that of the shallow impurity concentration. Second, consistent with the properties described above, it was clear that the behavior of this defect could not be explained by effective mass theory. Since in 1977 it was assumed that an isolated impurity atom could not be responsible for such complicated behavior, it was thought the the defect must be composed of a donor atom ("D") which was part of some unknown complex ("X"). Although it is now known that the defect is in fact due to an isolated substitutional impurity, the name has remained.

The first significant step in explaining the properties of these defects was made by Lang and Logan (1977). They suggested that the behavior exhibited by DX centers could be explained in terms of a large lattice relaxation. To make clear how a lattice relaxation can describe the properties of a large Stokes shift, a small capture crosis section, and persistent photoconductivity, it is useful to refer to a configuration coordinate diagram for DX centers, which is shown in Figure 1.2. Looking at the figure, it is clear that the DX center can be 


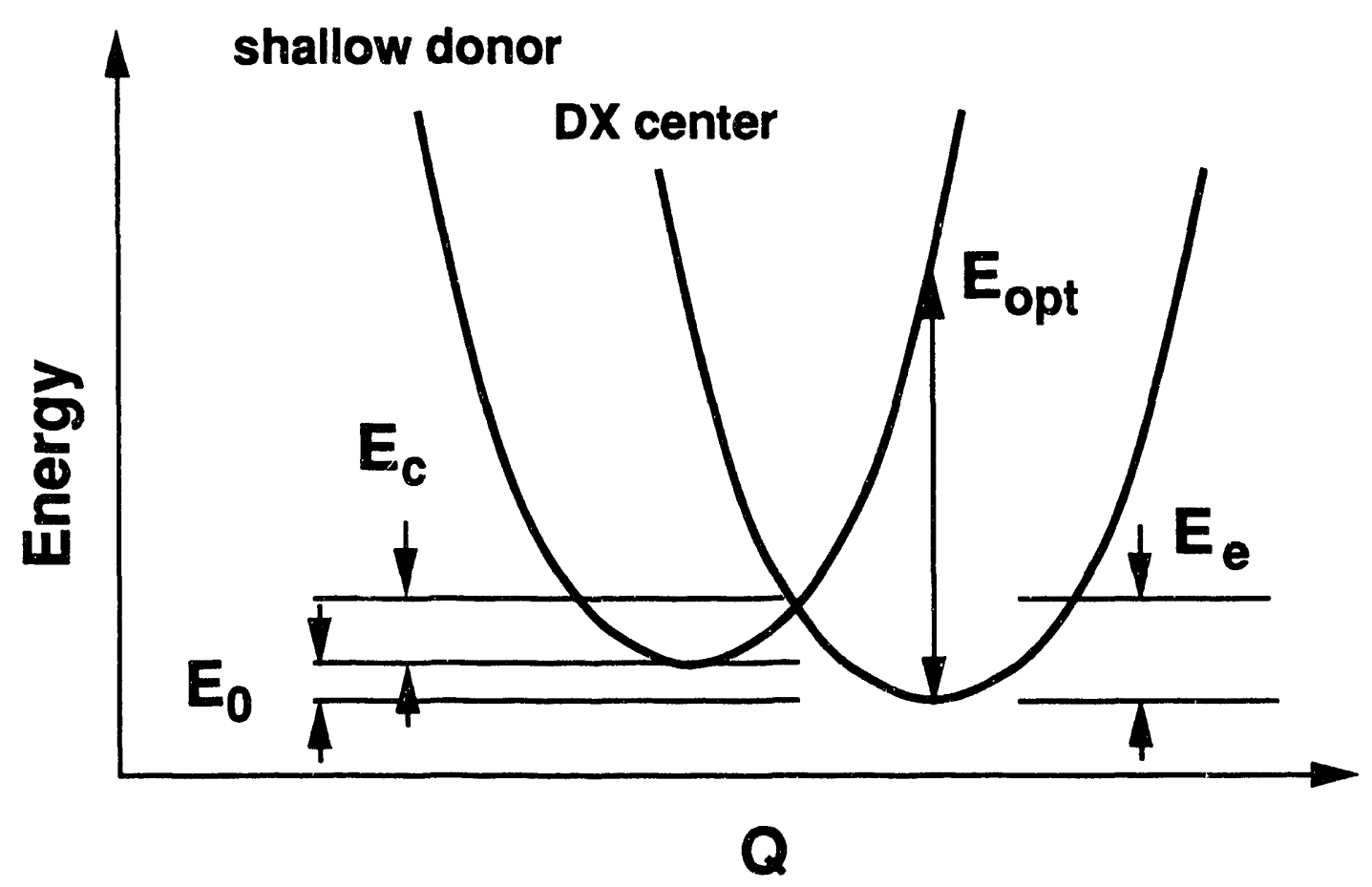

Fig. 1.2 Configuration coordinate diagram for DX centers. 
characterized by four energies: 1) $E_{0}$, the binding energy of the center relative to the shallow donor state, 2) $E_{e}$, the thermal energy required to emit an electron from the $D X$ to the shallow state, 3) $E_{o p t}$, the optical energy required to emit an electron from the deep to the shallow state, and 4) $E_{c}$, the capture barrier energy from the shallow into the deep DX state. We notice that, in contrast to Figure 1.1, the lattice relaxation is larger here and there will be no photoluminescence transition. In this case $d_{F C}=E_{o p t}-E_{0}=S h \omega$. Since the only difference between the shallow donor parabola and the conduction band parabola is a very small vertical offset, these two curves will not be differentiated in the remainder of this thesis. In fact, when the electron concentration is high, the impurity levels form a band which broadens and subsequently merges with the conduction band. In this case there is no difference between the two parabolas.

Fig. 1.2 illustrates the origin of the difference between the thermal and optical ionization energies of the DX center. The diagram also illustrates the origin of persistent photoconductivity. If the center is optically excited at low temperature, the electrons do not have sufficient energy to overcome the capture barrier and return to the deep DX state. It is interesting to note that it is not possible to optically excite an electron from the shallow level to the deep DX state. This could imply that the DX center parabola is more highly curved in the region approaching the unrelaxed donor configuration, and that the optical ionization energy would therefore be extremely large.

Finally, this diagram also can be used to understand the small capture cross section of DX centers. The capture of an electron is accompanied by a relaxation of the lattice. The lattice relaxation energy of the defect is simply $E_{o p t}-E_{0}$, i.e., the energy difference between the DX state and shallow donor state when the lattice is at the equilibrium configuration for the DX state. The 
relaxation is accomplished by the emission of phonons. Since this energy is so large $(\sim 1 \mathrm{eV})$, this implies that that a great many phonons are involved in the process and that it is therefore not very likely.

It was stated above that DX centers form in GaAs which has either been alloyed or put under hydrostatic pressure. This suggests that the formation of DX centers is tied to the structure of the conduction band since alloying with AlAs and applying hydrostatic pressure have similar effects on this band. One percent AlAs alloying is roughly equivalent to the application of $1 \mathrm{kbar}$ of pressure. Since the DX level is deep and must therefore be localized in real space, this implies that it is spread out in momentum space. This means that the derivative of the DX energy level with respect to alloying or pressure should be the same as that as the conduction band averaged over all $k$ space. The average conduction band rises in energy much more slowly than the conduction band minimum at $\Gamma$, which the shallow donor level follows. Therefore, at some percentage of alloying or under sufficient hydrostatic pressure the DX level becomes a more stable form of the donor than the shallow, substitutional form. This behavior is demonstrated in Figures 1.3a and 1.3b, which show how the DX level and various conduction band minima vary with alloying content and pressure in GaAs. The DX center becomes the most stable form of the defect at 20 percent AlAs content or about $20 \mathrm{kbar}$ of pressure.

One of the first important clues about the microscopic structure of the DX center came from an experiment which used local vibrational mode spectroscopy to demonstrate that the DX center is related to an isolated donor impurity (Maude, 1987). In this work, spectroscopy was used to show that in a sample of GaAs ninety percent of the Si was sitting substitutionally on the $\mathrm{Ga}$ site. The sample was then put under hydrostatic pressure sufficient to form DX 


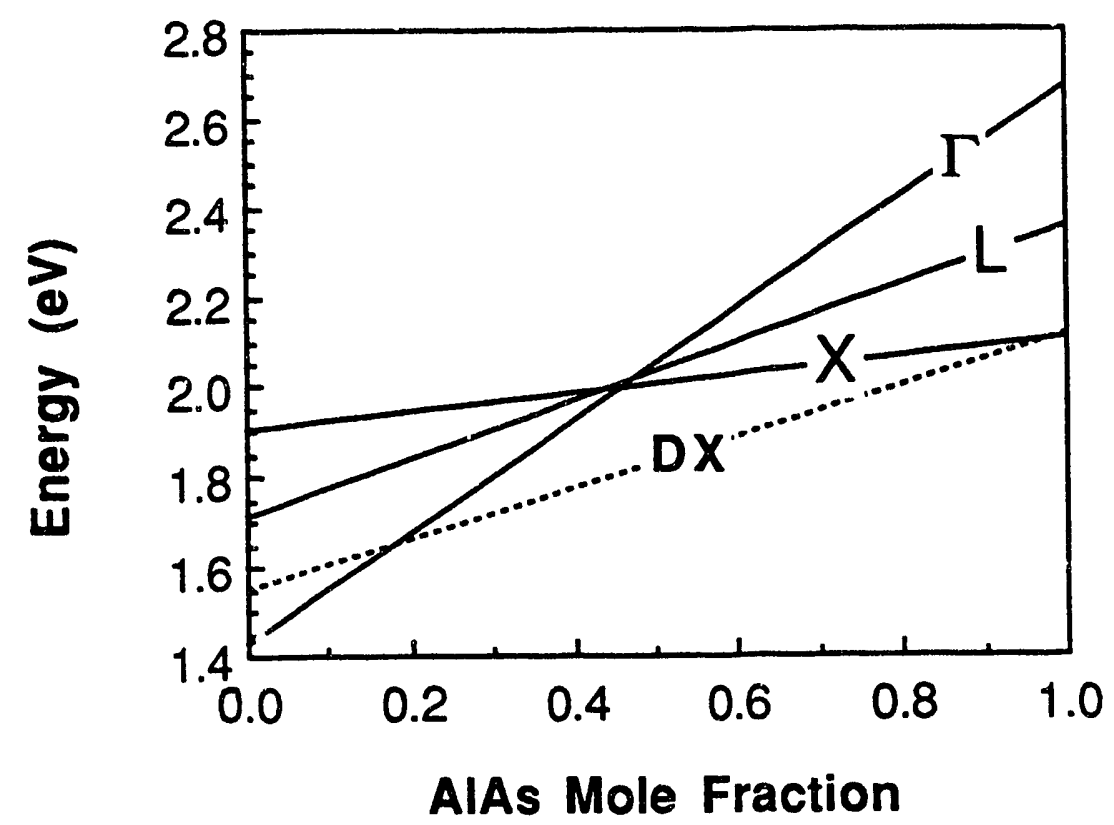

(a)

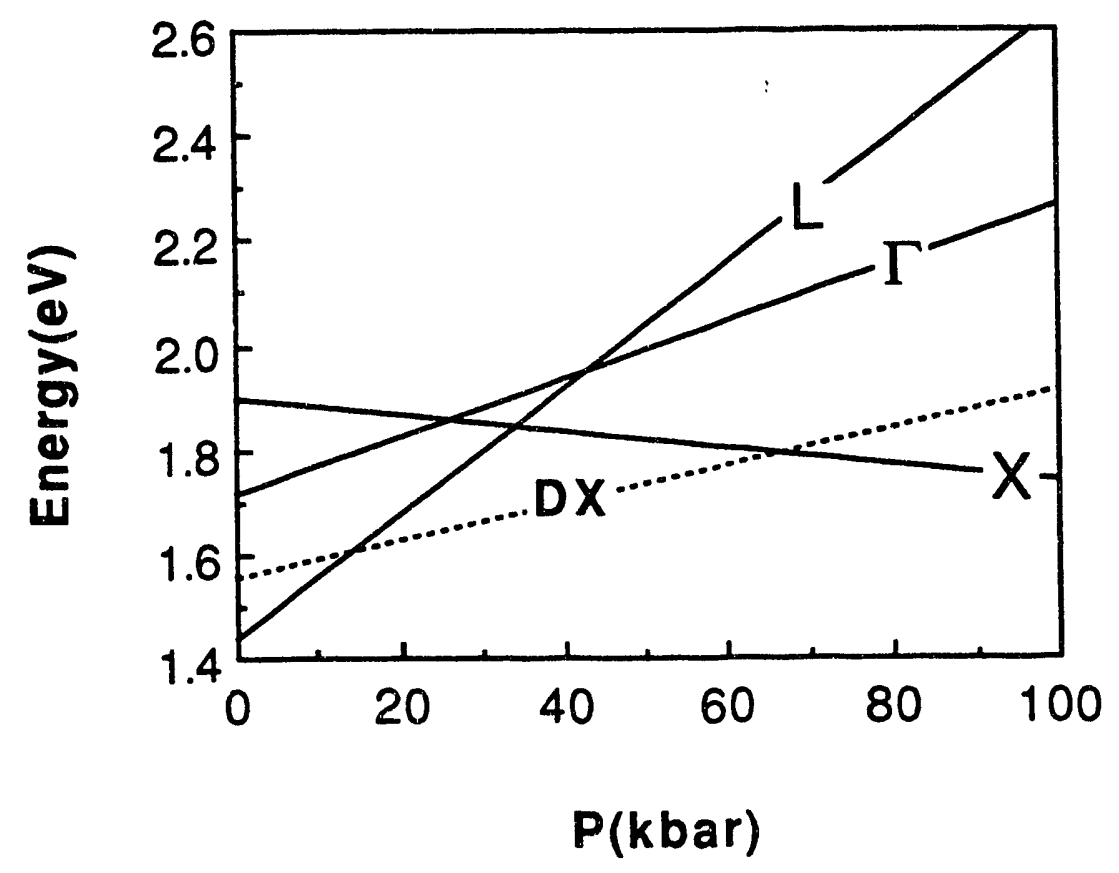

(b)

Fig. 1.3. Variation of the GaAs conduction band structure and the DX energy level as a function of (a) alloying with AIAs and b) pressure. 
centers, and it was found that the number of DX centers formed was roughly equal to the free-electron concentration due to the Si donors. Since it is extremely unlikely that simply applying pressure could cause a donor to form a defect complex, this made clear that the deep ievel was due to an isolated donor impurity.

There have been many subsequent experiments to further elucidate the microscopic structure of this defect. The two main questions to be answered are: 1) What is the charge state of the defect, and 2) What is the exact form of the lattice relaxation which occurs when the defect forms. A great deal of progress has been made in answering these questions on both experimental and theoretical grounds. Chapter 4 of this thesis describes an experiment which provides a definitive answer to the first question and important clues for the answer to the second question.

It is also important to know how general the formation of these defects is in III-V semiconductors. DX centers have been found in GaAs under pressure, AlGaAs, and GaAsP, but it is unclear whether or not this type of defect is a common feature in all III-V's, or if there is any method for predicting the conditions under which they might be observed. Chapter 5 of this thesis focusies on this question.

The bulk of the experimental results discussed in this thesis describes optical spectroscopy of DX centers in III-V semiconductors under high pressure. Chapter 2 will describe high pressure techniques in general, and Chapter 3 will describe how these techniques were coupled with optical analysis in order to study DX centers. 


\section{High Pressure Experimental Work Using Diamond Anvil Cells}

\subsection{Introduction}

Several different methods have been developed to obtain the high pressures required for different types of experimental work (Williams, 1991). These techniques can be divided into the categories of dynamic and static. The most commonly used dynamic method is the creation of shock waves to produce high pressures. In this technique, a high energy projectile is shot at a target containing the sample of interest. This creates stress waves moving faster than the speed of sound in the uncompressed material, resulting in shock waves which simultaneously stress and heat the target material. This technique is expensive and can only be used to study processes that occur on the order of a microsecond, but it is a well established method for producing high pressures and temperatures of many thousands of degrees Kelvin.

A static technique for creating high pressure uses large volume anvil cells. These cells are either of the piston cylinder type pictured in Figure 2.1a or the multi-anvil type shown in Figure 2.1b. Piston-cylinder cells are simply uniaxial-type stress rigs which create hydrostatic pressure through the containment of a pressure medium by the cylinder surrounding the piston. Multianvil cells create a more hydrostatic pressure environment than piston-cylinder cells and are also capable of providing higher pressures. However, they are difficult to design and build because of the simultaneous alignment required for all of the anvils. The main advantage of these two types of cells for static work is the possibility of containing a large sample volume. A disadvantage is the difficulty of incorporating optical access into their design. 


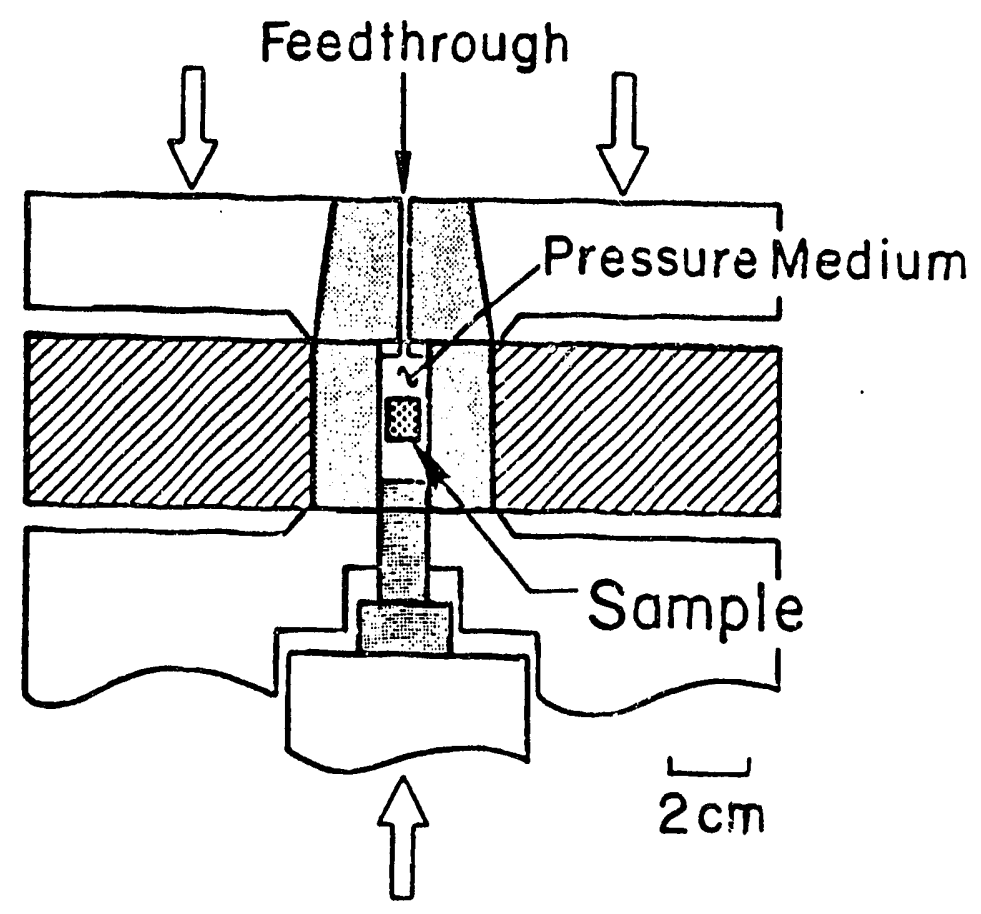

(a)

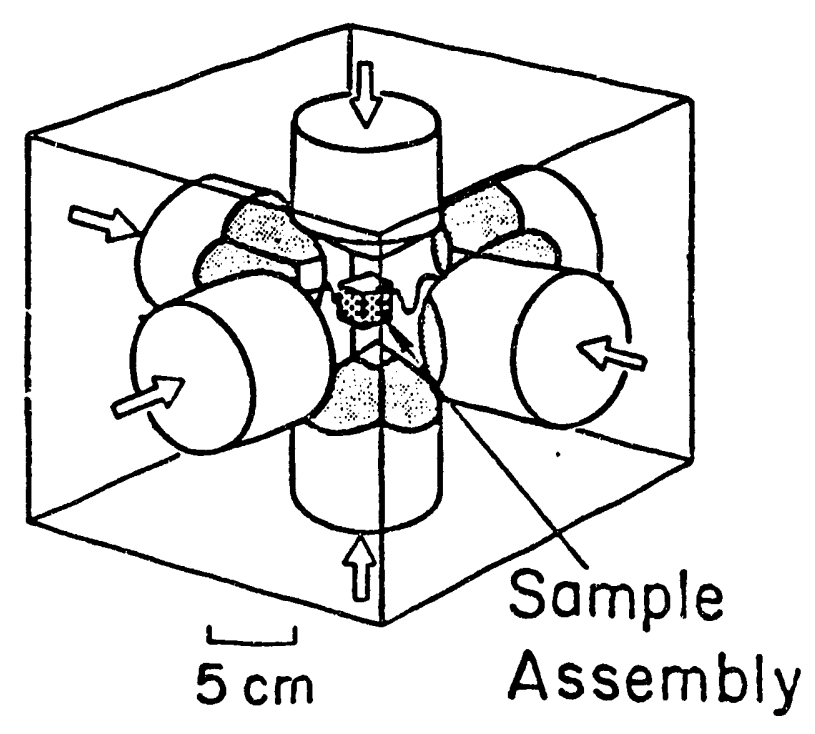

(b)

Fig. 2.1. Schematic illustration of large volume static high pressure techniques: a) piston-cylinder type cell, b) multi-anvil cell (E.K. Graham, 1986) design (Williams, 1991). 
Another static technique, and the one employed in the studies described in this thesis, makes use of diamond anvil cells. This technique has none of the shortcomings of the methods mentioned above. The central elements of a diamond anvil cell are shown in Figure 2.2. Two diamonds are held diametrically opposed with a piece of metal referred to as a gasket placed between them. A hole is drilled in the gasket to form the sample space, which is filled with the sample, a hydrostatic pressure medium, and small chips of ruby which are used for getermining the pressure. Pressure is applied by either a piston cylinder arrangement or three to six screws. These cells will be described in much more detail below. Diamond anvil cells are relatively inexpensive, simple in ciesign, and provide full optical access due to the transparency of diamond. This is of course the must critical feature for this work since it is comprised largely of optical spectroscopy. The major disadvantage of this technique is that one must use small samples, with maximum dimensions on the order of hundreds of microns.

The ranges of pressure and temperature for which the different techniques discussed here are useful are shown in Figure 2.3

\subsection{The Diamond Anvil Cell}

\subsubsection{Introduction to the Diamond Anvil Cell}

Diamond anvil cells (DACs) were first developed in 1959 (Jamieson, 1959; Weir,1959). The core of the cell consists of two diamonds which have had their culets ground down to form a flat surface a few hundred microns in diameter. The rest of the cell holds the diamonds diametrically opposed and 


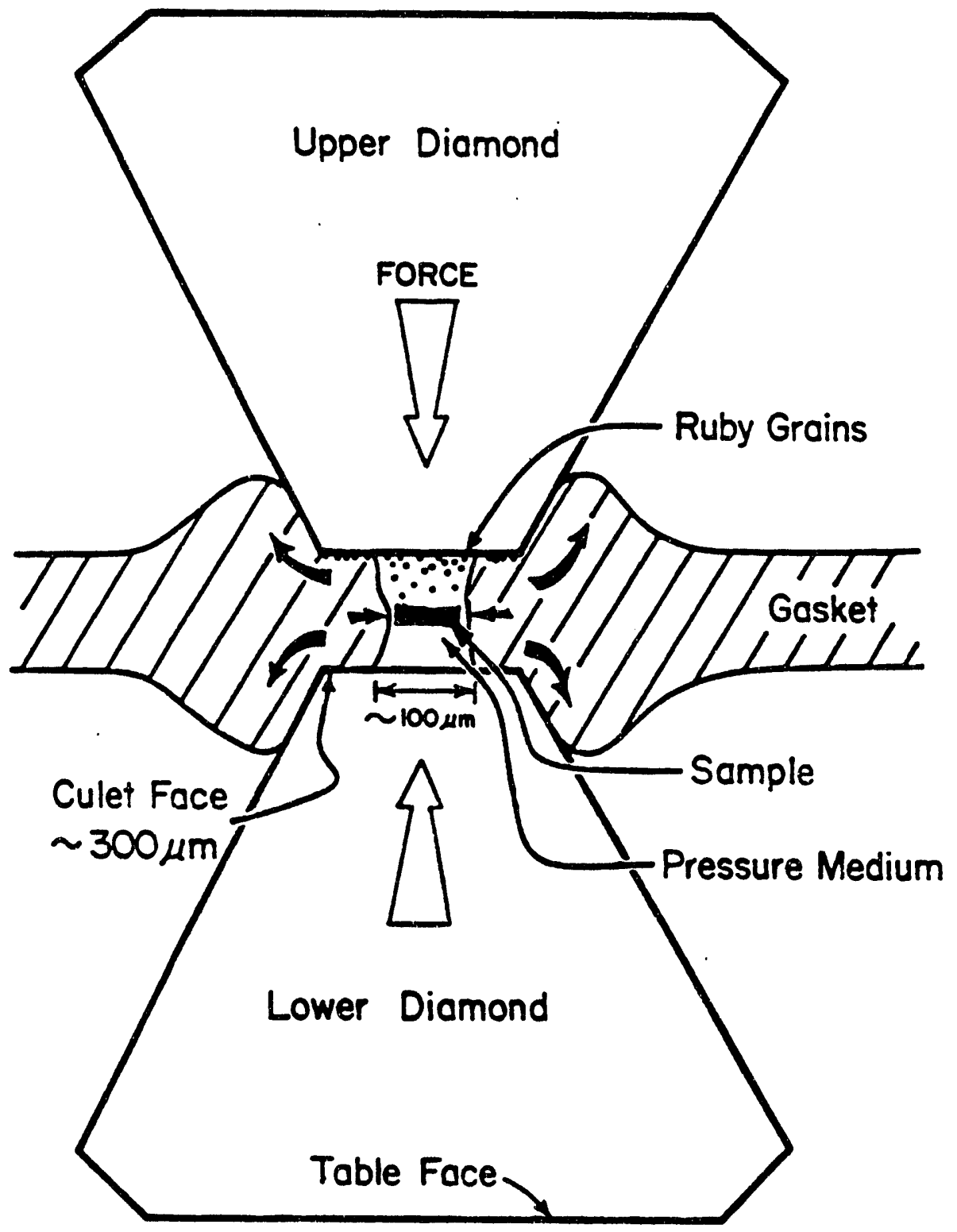

Fig. 2.2. Schematic cross-section of a diamond anvil cell iilustrating the way in which a sample is contained by a metal gasket while being squeezed between the culet faces of two diamonds. Ruby chips are used to measure the pressure (Williams, 1991). 


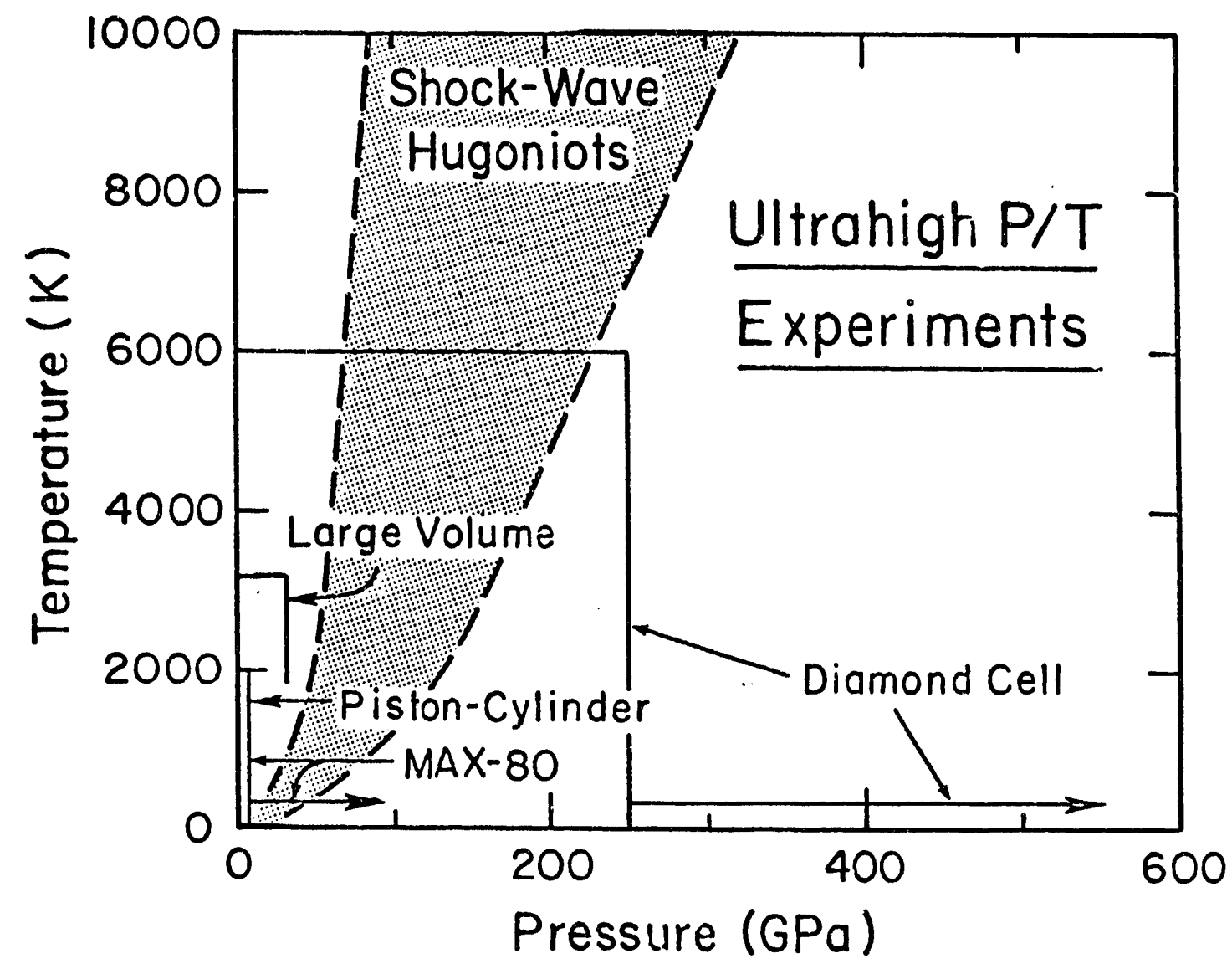

Fig. 2.3. Pressures and temperatures that can be achieved using various techniques discussed in the text. The shock wave temperatures depend on the sample material so the shaded area is only approximate. MAX-80 (Shimomura, 1985) is a specific multi-anvil cell (Williams, 1991). 
applies the force necessary to bring the diamonds together and exert pressure on the sample mounted between them. Diamonds are the best material for creating extremely high pressures because they are the hardest known material and are not prohibitively brittle. Diamonds are rated a 10 on the Mohs scale, and the interval between 9 and 10 represents a much larger difference in hardness than is normally represented by a single step on this scale.

In addition to applying pressure, there are two other important functions that a DAC must perform. First, it must keep the two diamonds translationally aligned. If this is not done, there will be areas on the face of the diamond which will have large pressure gradients, increasing the likelihood of diamond fracture. Second, the diamonds must be kept extremely parallel. If this does not occur, then the edge of one diamond will be forced into the face of the other, concentrating the stress at this edge and again making the probability of diamond failure high. Though there are several variations of diamond anvil cells, they differ mainly in the way that pressure is applied and fall into one of two categories: 1) piston-cylinder type or 2) Merrill- Basset type.

An example of the piston cylinder cell is the National Bureau of Standards cell designed by Piermiani and Block (1975) shown in Figure 2.4. Pressure is applied by turning a screw. and compressing Belleville washers. The upper diamond plate can be translated by the use of three adjustment screws symmetrically situated around the backing plate. The diamonds can be made parallel by tilting the hemispherical mount using the adjustment screw shown in the figure. The diamond mounts are normally made of metal which has been hardened to Rockwell 55-60 and have holes through their center to allow optical access for experimental purposes. Pressures as high as $500 \mathrm{kbar}$ have been reached with these cells, and pressures of several megabar have been reached with other piston-cylinder type cells (Jayaraman, 1983). 


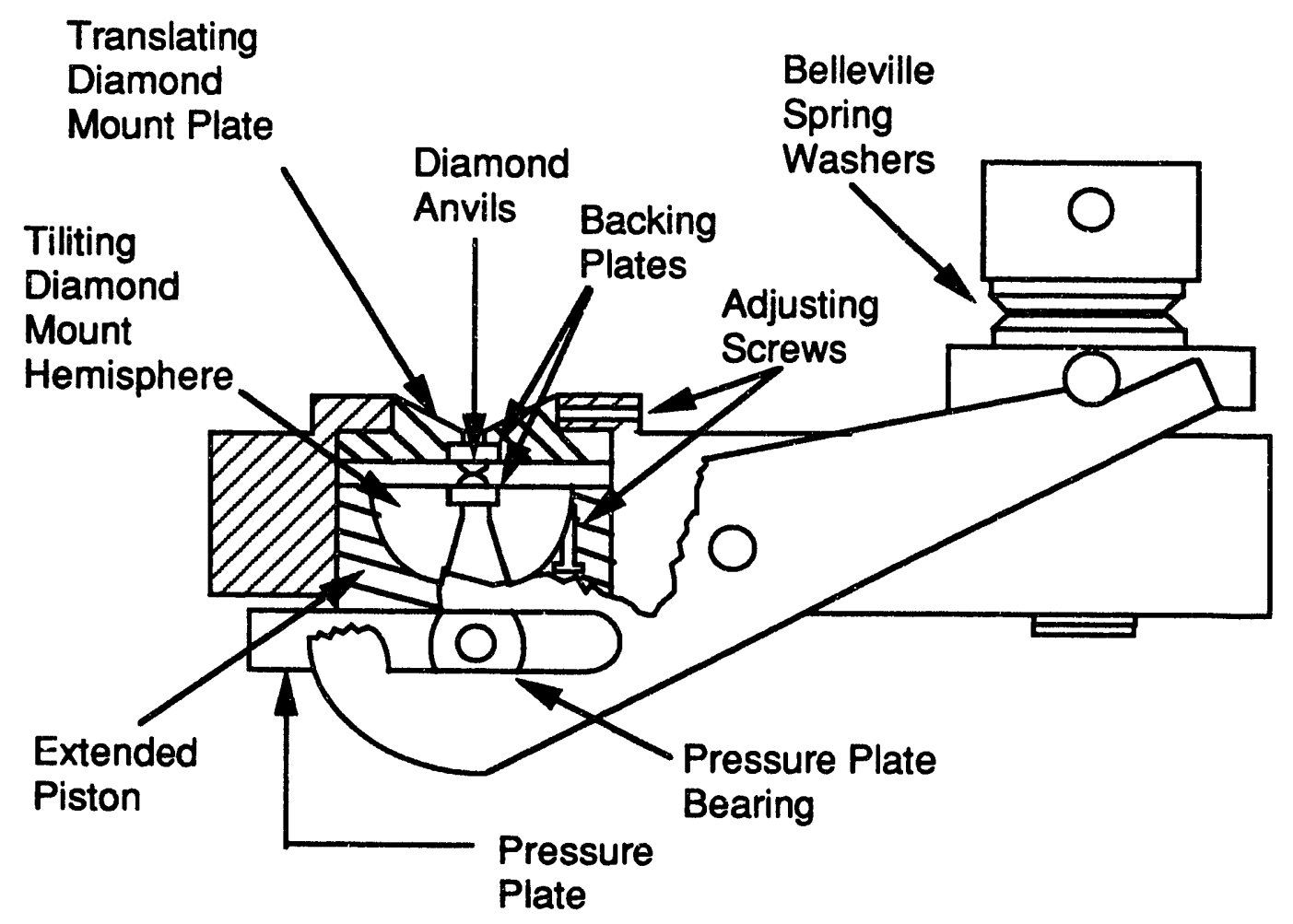

Fig. 2.4. Sectional view of the National Bureau of Standards ultra-high pressure DAC developed by Piermiani and Block (1975) (Jayaraman, 1983). 
The DAC used in the research for this thesis, shown in Figure 2.5 was designed by Sterer, Pasternak, and Taylor (1990). It is a Merrill-Basset type cell. In this type of DAC, pressure is applied by either three or six screws which pull the two halves of the cell together when tightened. The disadvantage of a Merrill-Basset type cell is that it is more difficult to keep the diamonds parallel than when using a piston-cylinder type cell, where alignment is automaticlly maintained. Alignment is maintained in a Merrill-Basset cell by measuring its thickness at three points around the cell diameter and tightening the screws in such a manner as to keep the relative thicknesses constant. Three pins coming up from the bottom of the cell mate with three holes in the top of the cell in order to help maintain this alignment. Translational alignment is performed using three adjustment screws situated symmetrically around the bottom backing plate. The upper backing plate is machined to fit very tightly into the hole where it is held in and is not free to move. The main advantage of this type of cell is that it can be made very small. In fact, the cell shown above is the smallest diamond anvil cell currently in use. Its diameter is slightly less than one inch, which allows it to fit into most cryostats and made the present work possible. This cell has been used to obtain pressures in excess of three hundred kilobar.

\subsubsection{Diamonds}

The type of diamond one chooses to use in the diamond anvil cell depends on the experiment to be performed. Diamonds are classified as either Type I or Type II. Type I diamonds are characterized by the presence of nitrogen impurities. The nitrogen forms several types of defects (Seal, 1984), including substitutional nitrogen, A centers (which are believed to be nitrogen pairs), B centers (which are believed to be larger nitrogen aggregates), and defects 


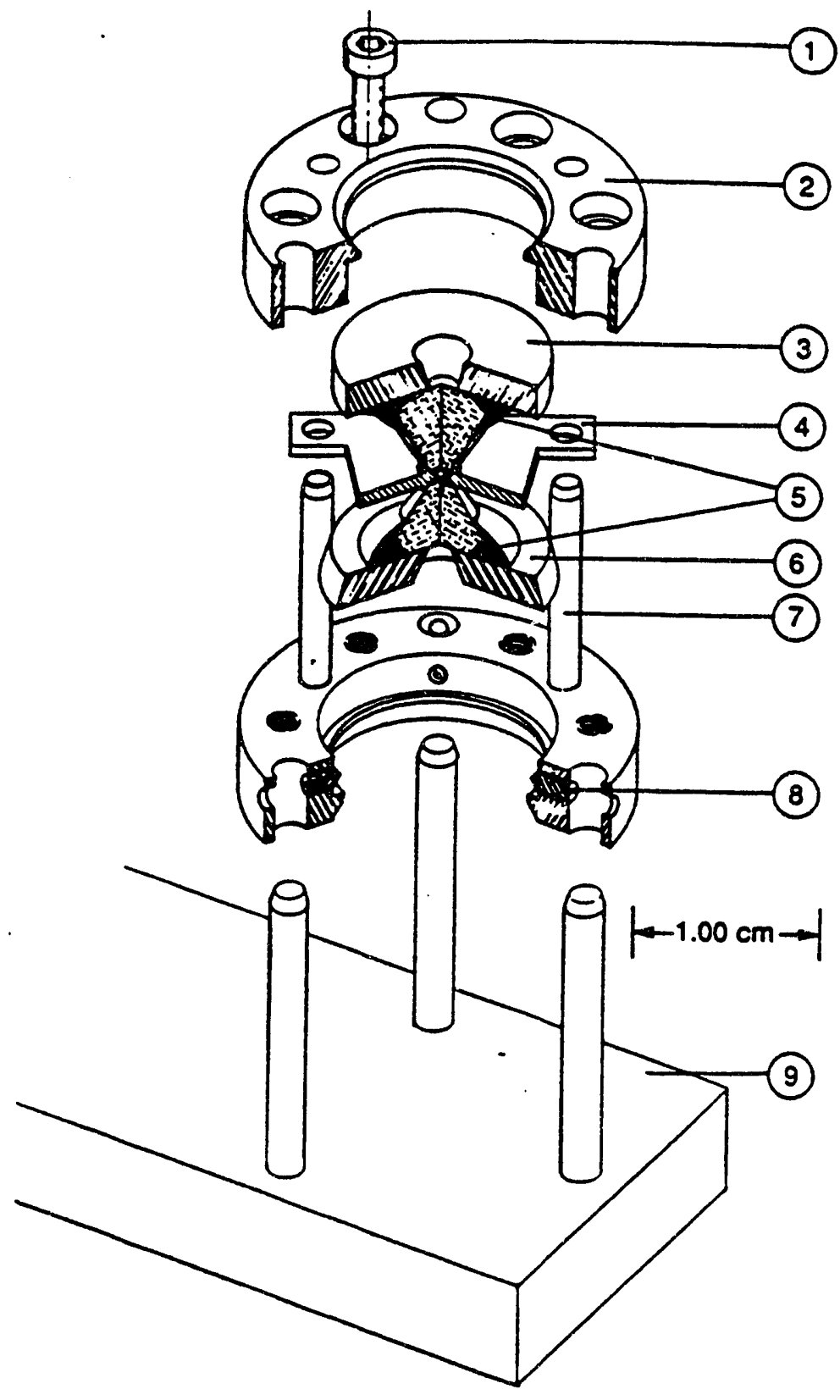

Fig. 2.5. An exploded view of the miniature diamond anvil cell: 1) Allen screws used to generate pressure, 2) platens, 3) upper fixed backing plate, 4) gasket, 5) diamond anvils, 6) lower adjustable backing plate, 7) positioning and gasket holder pins, 8) lower backing plate adjustment screws, 9) cell holder used to change pressure (Sterer, 1990). 
which are referred to as platelets because they were at first thought to be planar nitrogen defects. Although the platelets are believed to be nitrogen related, their precise structure is at present unknown. There are further subclassifications of these two categories, but these distinctions are not important for this work. The important point is that the four defects mentioned above have strong absorptions between 1100 and $1400 \mathrm{~cm}^{-1}$, making optical spectroscopy in this region impossible. Type II diamonds are all those diamonds which are not Type I; that is, they contain virtually no nitrogen. Since these diamonds represent only about $0.5 \%$ of the total of diamonds mined, they are much more expensive. Therefore, one chooses Type II diamond for optical work in the $1100-1400 \mathrm{~cm}^{-1}$ region, and Type I diamonds otherwise. All the work to be performed in this thesis was carried out using Type I diamonds.

Diamonds can be further classified by the way they are cut. There are two main types of cuts used in diamond anvil cell research. The first is shown in Figure 2.6a and is called the modified brilliant design. As the name suggests, it is a simple modification of the cut most commonly used to make jewelry. The modification is that the bottom of the diamond is truncated in order to form the culet face, which is the narrow flat section of the diamond. The larger flat section is referred to as the table, and the girdle is the thickest section of the diamond. This design was chosen by early workers in the field because it was readily available, but it is not the strongest possible cut. The brilliant design maximizes internal reflection and dispersion rather than strength.

The second, stronger design is referred to as the standard design and is shown in Figure 2.6b. The main goal of this design is to maximize the amount of the diamond which is in the volume directly beneath the diamond table. The fraction of the diamond outside of this volume is under low stress in a DAC and does not contribute very much to the strength of the anvil (Seal, 1987). In fact, in 

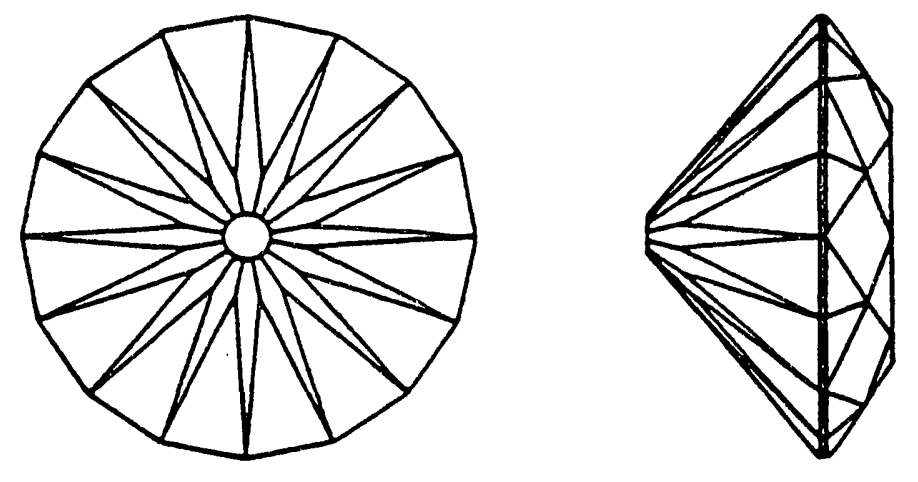

(a)
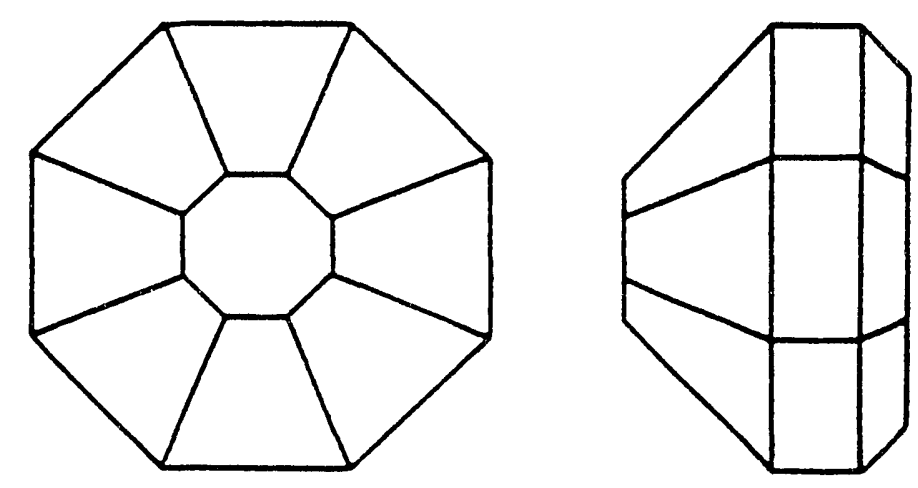

(b)

Fig. 2.6. Shapes of diamond anvils: a) modified brilliant design, b) standard design (Seal, 1987). 
the modified brilliant design, only $57.5 \%$ of the diamond is in the critical volume. It is clear from looking at the figure that the standard design is much more efficient in this respect. This is accomplished by reducing the number of facets form fifty to twenty-six, drastically thickening the girdle, and lowering the table towards the girdle. Diamonds of the standard design were used for the most of the work described in this thesis. It should be mentioned that to achieve very high pressures ( $>500 \mathrm{kbar}$ ), the culet is often beveled. This reduces the stress concentrations on the edges of the culet.

Low birefringence is another important factor in choosing diamonds. Since diamond is a cubic lattice, if it were unstrained it should have no birefringence. The current belief is that a diamond with low birefringence is less strained and is therefore less likely to fail under the extreme conditions to which anvils are subjected.

\subsubsection{Gaskets}

When diamond anvil cells were first introduced, the sample was simply squeezed between the two diamond anvils, which of course created a uniaxial stress on the samples. To achieve high pressure it is necessary to use hydrostatic pressure, and this necessitates the use of a gasket. A gasket is a thin piece of metal placed between the two diamonds with a hole drilled in it in order to form a sample space. Its use was first demonstrated by Van Valkenburg (1965). The hole is filled with a pressure medium, a sample, and chips of ruby for measuring pressure (this will be discussed in detail in Section 3.3). The sample space is sealed by bringing the top diamond down onto the assembly of the bottom diamond plus gasket. 
Materials commonly used for gaskets include Inconel, spring steel, and for very high pressures, rhenium. The initial thickness of the gasket is usually roughly $250 \mu \mathrm{m}$ and it is indented to a thickness of approximately $50-100 \mu \mathrm{m}$. The indentation of the gasket is shown in Figure 2.2, which also illustrates how the gasket material extrudes around the diamond faces. It is thought that the extruded material helps to prevent diamond failure hy supporting the anvils near the edges where stresses may concentrate.

\subsubsection{Pressure Media}

A wide variety of pressure media have been used for DAC research, and Table 2.1 gives a list of some of these materials along with their useful pressure ranges at room temperature. A 4:1 mixture of methanol:ethanol is perhaps the easiest on the list to work with because it is a liquid at room temperature and no cryogenics are required. However, a strong disadvantage of this medium from the point of view of spectroscopy is that it absorbs in the infrared. This problem can be overcome by using liquid $\mathrm{N}_{2}$ and $\mathrm{Ar}$, both of which are transparent in the infrared. Although these are cryogenic liquids, it turns out that loading cells with them does not present too much extra difficulty. The most hydrostatic medium to use is liquid $\mathrm{He}$, but this requires sophisticated apparatus for the loading of samples. All work in this thesis used either methanol:ethanol or liquid nitrogen as the pressure medium, and the techniques for loading cells using these media will be discussed in the next section. 
Table 2.1. Some pressure media and their useful pressure ranges at $300 \mathrm{~K}$ (Jayaraman, 1983).

Medium

Methanol:Ethanol

4:1 Methanol:Ethanol

$\mathrm{He}$

$\mathrm{Ne}$

Ar

$\mathrm{Xe}$

$\mathrm{H}_{2}$

$D_{2}$

$\mathrm{N}_{2}$

$\mathrm{O}_{2}$
Pressure Limit for nearly hydrostatic behavior (kbar)

$\approx 200$

$\approx 200$

$>600$

160

90

300

$>600$

?

130

? 


\subsubsection{Loading the Diamond Anvil Cell}

In this section I will discuss how to load the miniature DAC described earlier. The first step in loading this or any diamond anvil cell is to glue the diamonds to the backing plates. The epoxy to be used is determined by the type of experiment being performed. For the low temperature work described in this thesis, it was found that both Stycast 2850 and Miller-Stephenson 907 epoxies worked well, meaning they would adhere to the metal backing plates and diamonds and remain stuck through many thermal cyclings between room temperature and $4.2 \mathrm{~K}$.

Once the diamonds are glued, they must then be aligned. The first step in aligning is to roughly center the bottom backing plate using the three set screws in the bottom the cell. The top plate (with diamond glued on) is pushed firmly into the top half of the cell. It is important to make sure that both backing plates are lying completely flat, since otherwise the high pressure encountered in the loading process will make them lie flat and change the relative tilt of the diamonds.

The next step is to insure that the diamonds are parallel. After sliding the top half of the cell down onto the bottom half, which should be done very gently, one observes interference fringes between the diamond culets. These fringes are simply an example of Newton's rings. By gently tilting the cell, one watches for the disappearance of the rings to signal that the culets are parallel. At this point three screws should be put in and tightened so that the diamonds are snug.

The diamonds must next be aligned translationally. This is done using the three set screws which were used to roughly center the bottom backing plate above. They are adjusted until the faces line up. If using standard cut 
diamonds, which have only eight sides, it is important to make certain that the octagonal faces line up at each vertex. This usually requires rotation of the bottom diamond. The culets of the sixteen-sided modified brilliant cut diamonds are so close to circular that rotational alignment is not required.

After the translational alignment is complete, it is likely that the diamond will no longer be precisely parallel. So, the procedure for making them parallel must be repeated, followed by anoiher translational alignment. This procedure is iterated until the culets appear to be completely aligned. At this point, the thickness of the cell should be recorded at each of the three screw positions. These relative thicknesses should be maintained at all times when working with the cell. The three thicknesses should all agree to within $0.1 \mathrm{~mm}$. If they don't, there is probably some foreign matter underneath the backing plates which should be removed. After the cell is aligned, the gasket must be prepared.

A gasket should be cut out in the shape shown in Figure 2.7. Three holes are drilled in order to slip over the three alignment posts of the cell, which makes it possible to slip the gasket on and off and continually place it in precisely the same position. Most of the material must be cut away to make room for the pressure application screws.

The next step is to pre-indent the gasket, which is done by first slipping the gasket onto the bottom half of the cell. The top of the cell is then put on and three screws are put in place and slowly tightened, measuring the cell thickness at the specified points to insure the maintenance of culet parallelism. The screws are tightened until the gasket has the desired thickness. In addition to the advantages of pre-indentation mentioned in the section on gaskets, it is also believed by some workers in the field that the diameter of the sample hole is less likely to increase under pressure if the gasket material is thin. If the hole diameter does increase, the pressure medium will leak out when the hole 


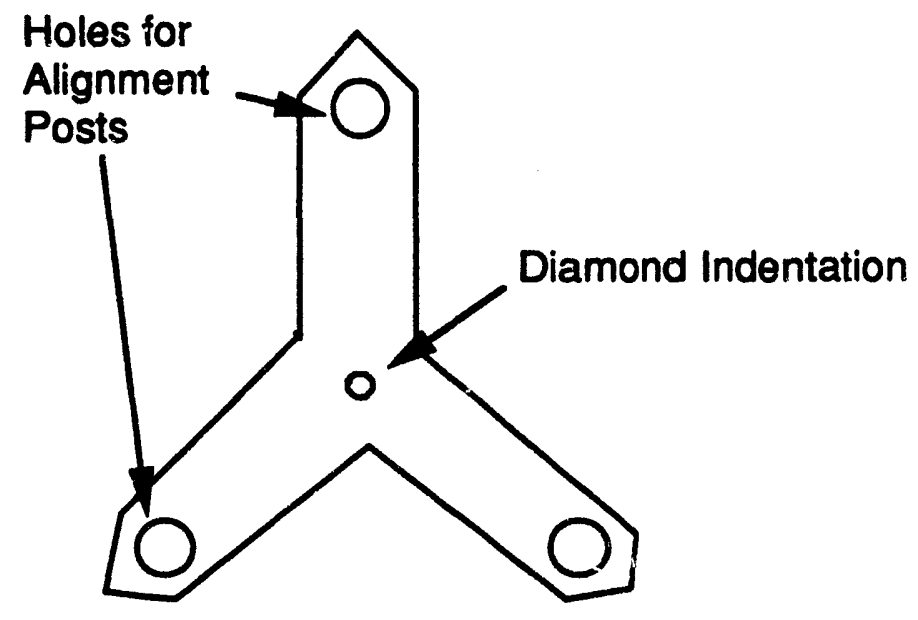

Figure 2.7. Optimum shape for gaskets. The diamond indentation is made in the middle of the gasket. 
reaches the edge of the diamond culet. Of course, the gasket must not be made thinner than the sample. A further advantage of pre-indentation is that it allows one to drill a sample hole which will sit in the middle of the diamond faces, and it also helps in putting the gasket in precisely the same position each time.

After pre-indentation is complete, pressure is slowly released and the gasket is removed from the cell in order to drill a hole to form the sample space. A \#78 tungsten carbide drill bit was used to drill a $400 \mu \mathrm{m}$ hole to contain a 300 $\mu \mathrm{m}$ sample for much of the work described in this thesis. One has to start with a hole bigger than the sample diameter because the hole size initially decreases with increasing pressure. Once the hole is drilled and deburred, the thickness of the pre-indented portion of the gasket can be measured. If it is not of the desired thickness, further pre-indentation can be carried out. Once the gasket is prepared the cell can be loaded

Regardless of the pressure medium which is used, the first steps in the actual loading of a sample are the same. The gasket is placed in position and the sample, along with a few chips of ruby which have been ground to a few microns in diameter, are placed in the sample space. The bottom half of the cell is then placed on a tightening stage, which is simply a piece of aluminum with three posts which mate with three holes in the cell. This allows one to tighten the cell without having it rotate.

The remainder of the procedure is dependent on the pressure medium used. In order to load with a methanol:ethanol mixture, a syringe is used to put one or two big drops of the medium onto the sample. To avoid evaporation, the top of the cell must be brought quickly down and the screws tightened by going around the diameter of the cell turning each screw a small amount. Six pressure application screws are used if the desired pressure is above $\mathbf{5 0} \mathrm{kbar}$, otherwise three screws are sufficient. 
In order to load with nitrogen, the top half of the cell is mounted and the pressure application screws are put in. However, no force is applied. The screws are tightened just to the point before any force is required, and they are adjusted until measurements of the relative thickness around the cell verify that the diamonds are aligned. The entire cell is then put on the tightening stage and immersed in liquid nitrogen. After the nitrogen has stopped boiling rapidly, helium gas is bubbled in. This reduces the boiling tremendously, making it much less likely that nitrogen gas will be trapped in the sample space. If gas is trapped, the hole will collapse inwards when pressure is applied and crush the sample. It is not clear why bubbling in the He gas is so helpful, but it is hypothesized that the gas increases the thermal conductivity of the nitrogen, which would mean that the heat would be brought to the surface much more quickly and would subsequently decrease the boiling rate. Once the nitrogen is sufficiently calm, the pressure application screws are tightened by going around the diameter of the cell turning each screw a small amount. This procedure is continued until the screws can no longer be tightened using a screwdriver allen wrench. The cell is then removed from the liquid nitrogen.

\subsubsection{Sample Preparation}

There are two steps to preparing semiconductor samples for loading into a diamond anvil cell. The first step is thinning and polishing of the sample, which occur simultaneously, and the second step is cutting. As discussed in the previous section, it is thought that the diameter of the gasket hole is less likely to increase under pressure if the gasket material is thin. If the hole diameter does increase, the pressure medium will leak out when the hole reaches the edge of 
the diamond culet. This implies that the attainment of higher pressures requires the preparation of thinner samples in order that they will not be crushed by a thinner gasket. The desired sample thickness is normally in the range 25-100 $\mu \mathrm{m}$, which corresponds to a maximum pressure between roughly 115 and 40 kbar, respectively.

The first step in thinning and polishing is to use a dicing saw to cut out a small piece of semiconductor 250-375 $\mu \mathrm{m}$ thick. Any attempts to cut thinner pieces of GaAs or InP will result in a shattered sample. GaAs samples may be then lapped by hand using a $3 \mu \mathrm{m} \mathrm{Al}_{2} \mathrm{O}_{3}$ slurry as long as the samples do not need to be thinner than $50 \mu \mathrm{m}$. Thinner samples are too fragile and must be mounted on some form of lapping plate, which is often simply a glass slide. A finer polish can be produced on GaAs samples by a final polish on a felt pad saturated with bleach. The preparation of very thin InP samples $(\sim 25 \mu \mathrm{m})$ requires more care because these samples are extremely fragile. They must be lapped on a graphite block so that after they are cut, the wax used to hold them in place can be dissolved from behind the sample. This allows one to dismount the samples without having to handle them very much. All sample movement is accomplished with the bristles of a very fine paint brish.

In Fourier transform spectroscopy it is important to minimize the amount of light leaking around the sample. Since the sample hole in the gasket is round, it was decided that round samples should be cut to fill the hole. In order to perform the delicate task of cutting cylindrical shaped samples $300 \mu \mathrm{m}$ in diameter and $\sim 50 \mu \mathrm{m}$ thick, a tip for a low power ultra-sonic cutting machine was made which used the flattened end of No. 23 hypodermic needles as the cutting surface. Do-All wax was the only wax found that would consistently hold the samples to either the glass slide or piece of graphite to which the sample was mounted. Sticky wax was somewhat less effective but also proved useful. 
With this technique, a high percentage of undamaged samples could be recovered after cutting. After recovery, the samples are boiled in TCA and methanol to remove wax and grease.

\subsection{Pressure Measurement}

\subsubsection{The Ruby Fluorescence Scale}

The ruby fluorescence scale for measuring hydrostatic pressure was developed by Forman (1972) and is based on the pressure dependence of the wavelength of the $R_{1}$ and $R_{2}$ fluorescence lines of the $C^{3}+$ ion in ruby. These lines may be excited with blue or green light from a $\mathrm{He}-\mathrm{Cd}$ or $\mathrm{Ar}$ ion laser. The doublet structure of the fluorescence is a result of the size of the $\mathrm{Cr}^{3+}+$ ion. In a cubic field the $\mathrm{Cr}^{3+}$ ion would show only a single fluorescence line due to the spin transition ${ }^{2} \mathrm{E} \rightarrow{ }^{4} \mathrm{~A}_{2}$. However, since the $\mathrm{Cr}^{3}+$ replaces an $\mathrm{Al}^{3+}$ atom and is too big to fit substitutionally, it assumes a lower symmetry position and the ${ }^{2} E$ is split due to a removal of spin-orbit degeneracy (Ferraro,1984). Pressure measurements based on this doublet are problematic at temperatures above $300{ }^{\circ} \mathrm{C}$ where the two lines broaden into one. However, ruby has been found to be the overall best pressure calibrant because 1) its fluorescence is more intense than other materials, 2) the pressure dependence of the fluorescence lines is reasonably strong, and 3 ) the $R$ linewidth is extremely sharp (7.5 $\AA$ ) (Barnett, 1973).

The dependence of the transition wavelengths has now been calibrated out to one Megabar against the $p-V$ relationship of several materials, including Co, Mo, Pd, and $\mathrm{Ag}$ (Mao, 1978), as shown in Figure 2.8. This dependence is 


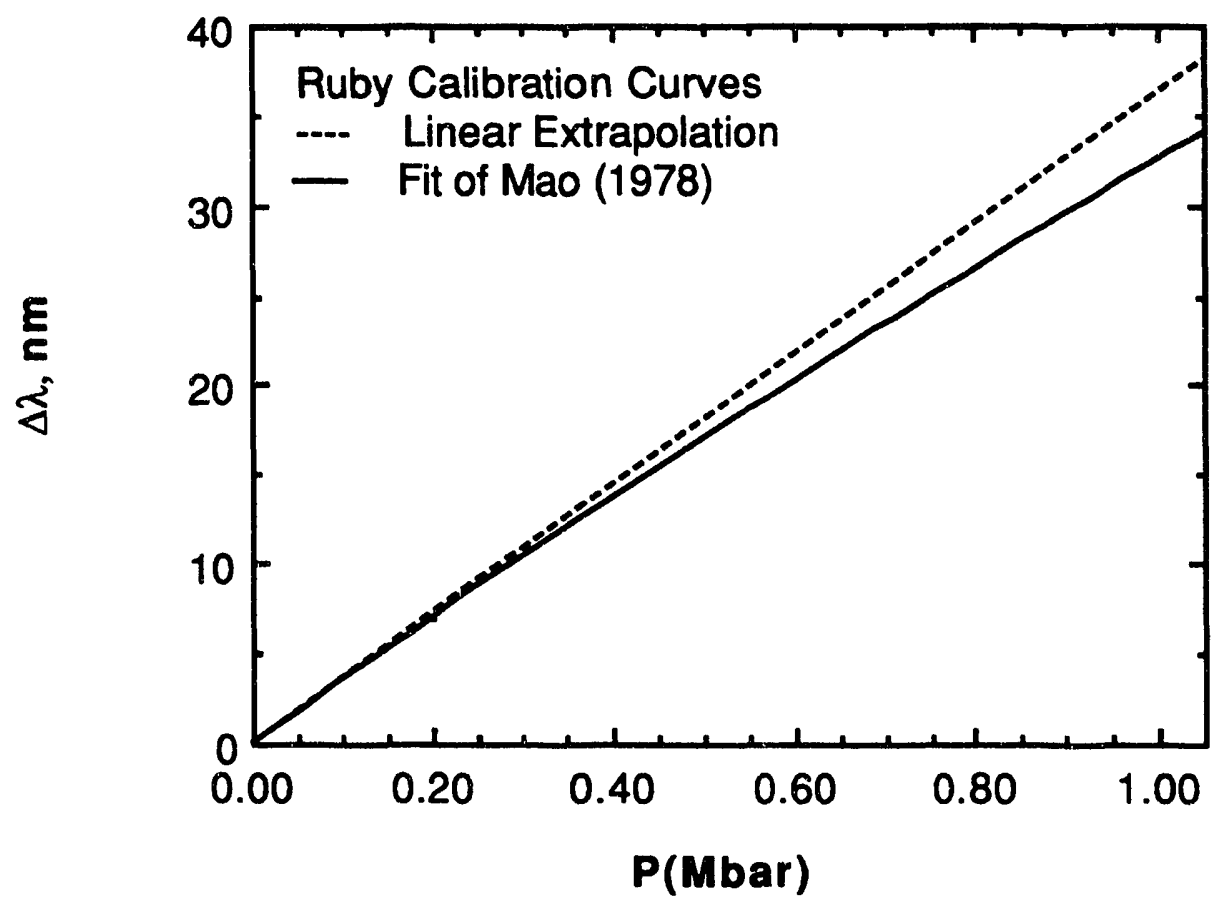

Fig. 2.8. Shift of $R_{1}$ and $R_{2}$ ruby fluorescence lines with pressure (Jayaraman, 1983). 
found to be linear below $300 \mathrm{kbar}$ and to have a magnitude of $0.36 \AA \AA / \mathrm{kbar}$. The dependence is sublinear at higher pressures and Mao has proposed a calibration curve of

$$
p^{\prime}(M b a r)=3.808\left((\Delta \lambda(\AA) / 6942+1)^{5}-1\right) .
$$

The hydrostatic pressure dependence of both lines in the doublet is found to be equal, and they could be used interchangeably for calibration purposes. The $R_{1}$ line is usually chosen though because it is slightly stronger.

\subsubsection{Pressure Measurement System}

The pressure measurement system which was constructed for this research is shown in Figure 2.9. The main part of the set-up is an Olympus microscope which is capable of magnifications ranging from 200 to 400 times. The main advantage of using a microscope is that it is easy to focus the laser beam onto any particular chip in the ruby cell. This set-up is much simpler than pressure measurement systems set up on an optical table since there are no adjustments of any optical components required. The focussing is automatically taken care of by the microscope.

A $25 \mathrm{~mW}$ Omnichrome $\mathrm{Ar}$ ion laser is used to excite the ruby fluorescence. The light from the laser is directed onto a dichroic mirror which reflects the blue-green laser light down to the diamond anvil cell but lets oniy the red fluorescence light travel up through the microscope and into the monochromator. The dichroic mirror is used to prevent any laser light, which could potentially destroy the photomultiplier tube used to detect the fluorescence, from entering the monochromator. The ruby fluorescence is then 


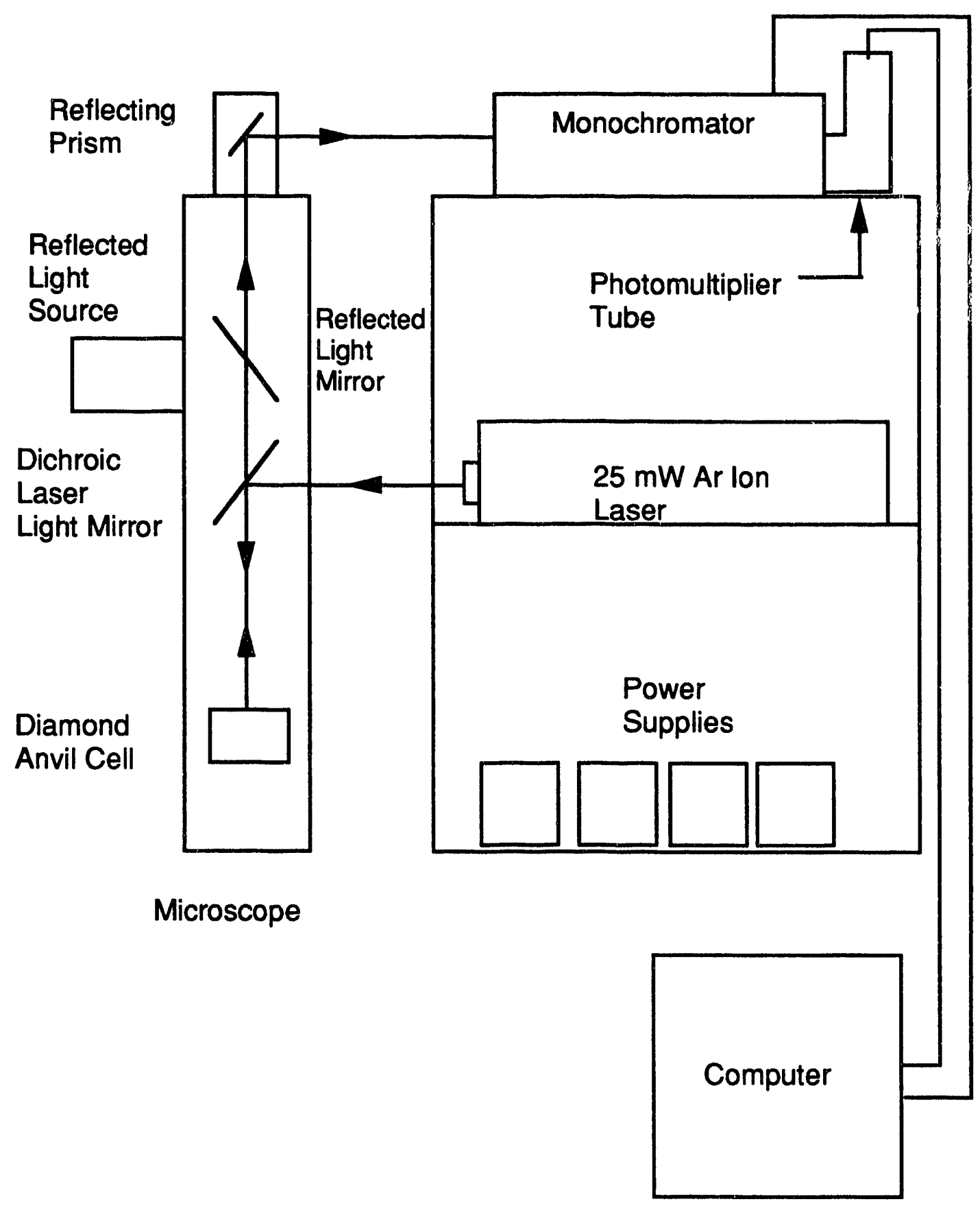

Fig. 2.9. Schematic of pressure measurement system. 
collected by the objective lens and travels back up through the microscope. At the top of the microscope, the light is reflected by a right angle prism into a Sciencetech 9050 monochromator, which is controlled by a Gateway 2000 $386 / 25 \mathrm{MHz}$ computer. The light is detected at the exit slit of the monochromator by a Hamamatsu 928 multialkalai photomultiplier tube which has a Hamamatsu 1506-01 pre-amplifier attached directly to its back end. The signal from the preamplifier is then fed into a A/D board plugged into the computer. Each time the monochromator steps, one hundred values from the A/D board at the given wavelength are averaged and the result then recorded. This allows one to record a spectrum of intensity as a function of wavelength, and shows the characteristic $R_{1}-R_{2}$ doublet if the lasur is focussed on a ruby chip. An example of such a spectrum is shown in Figure 2.10.

One complication in this work is that it was necessary to know the pressure in the DAC at low temperature, but it is not possible to perform ruby fluorescence while the DAC is at liquid helium temperature. The following technique was therefore employed to calibrate the pressure in the cell at low temperature. The pressure in the DAC was measured at room temperature, after which it was immersed in liquid nitrogen. The pressure was then measured again taking into account $d v / d T$ of the fluorescence, which is independent of pressure (Jahren, 1990). It was assumed that the pressure did not change between $77 \mathrm{~K}$ and $4 \mathrm{~K}$ since the coefficient of thermal expansion of the cell, which is made of Vascomax 350 , is small at low temperatures. It was found that the pressure in the cell at $77 \mathrm{~K}$ was roughly four kbar below the pressure measured at $300 \mathrm{~K}$ after the cooling process. This change in pressure is independent of the pressure in the cell. 


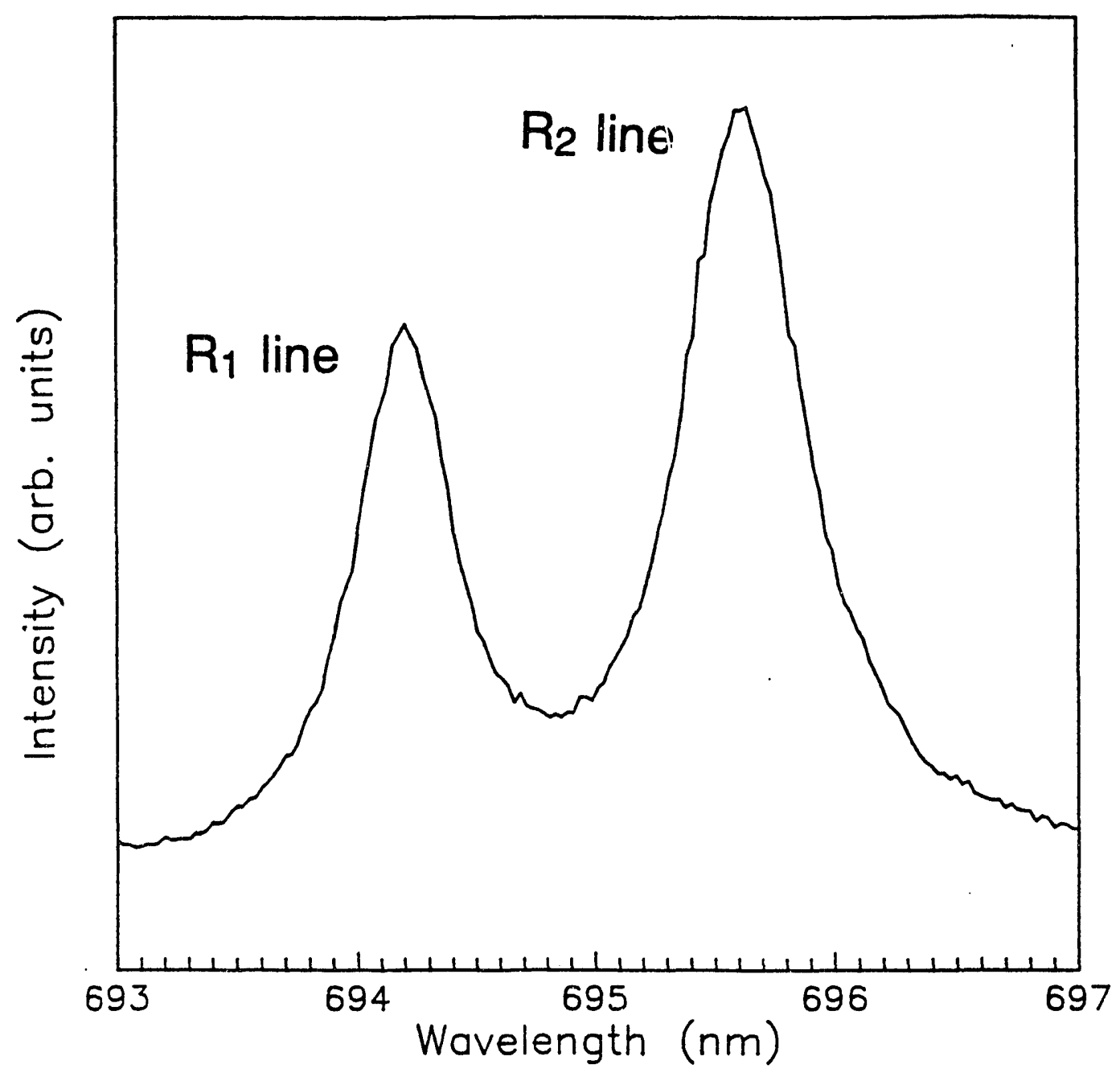

Fig. 2.10. Sample spectra of ruby fluorescence $R_{1}$ and $R_{2}$ lines taken with the pressure measurement system described in the text $(P=51$ kbar). 


\section{Characterization Techniques}

While the previous chapter described techniques for bringing semiconductor samples io high pressure, this chapter will focus on how these samples were characterized in the work described in this thesis. The main techniques used to study the samples were optical spectroscopy, employing both Fourier transform and grating spectrometers, and Hall effect. This chapter will provide an introduction to all of these techniques, including those used to perform optical spectroscopy under high pressures in a diamond anvil cell.

\subsection{Fourier Transform and Grating Spectroscopy}

\subsection{Fourier Transform Spectroscopy}

Spectroscopy of semiconductor defects comprises a major part of this thesis, so it is appropriate to give a brief discussion of the different types of spectrometers used. We begin with a description of a Fourier transform spectrometer. The heart of this instrument is a Michelson interferometer, shown schematically in Figure 3.1. It consists of a light source, a beamsplitter, a fixed mirror, a moving mirror, and a detector. The light travels from the source to the beamsplitter, where part of the beam is reflected to each of the mirrors. The two beams are then reflected back to the beamsplitter, where they recombine en route to the detector. In Fourier transform spectroscopy, the intensity of the light at the detector is measured as a function of the position of the moving mirror. The plot of this intensity versus position relationship is referred to as an interferogram. The desired result in a spectroscopy experiment is a spectrum 


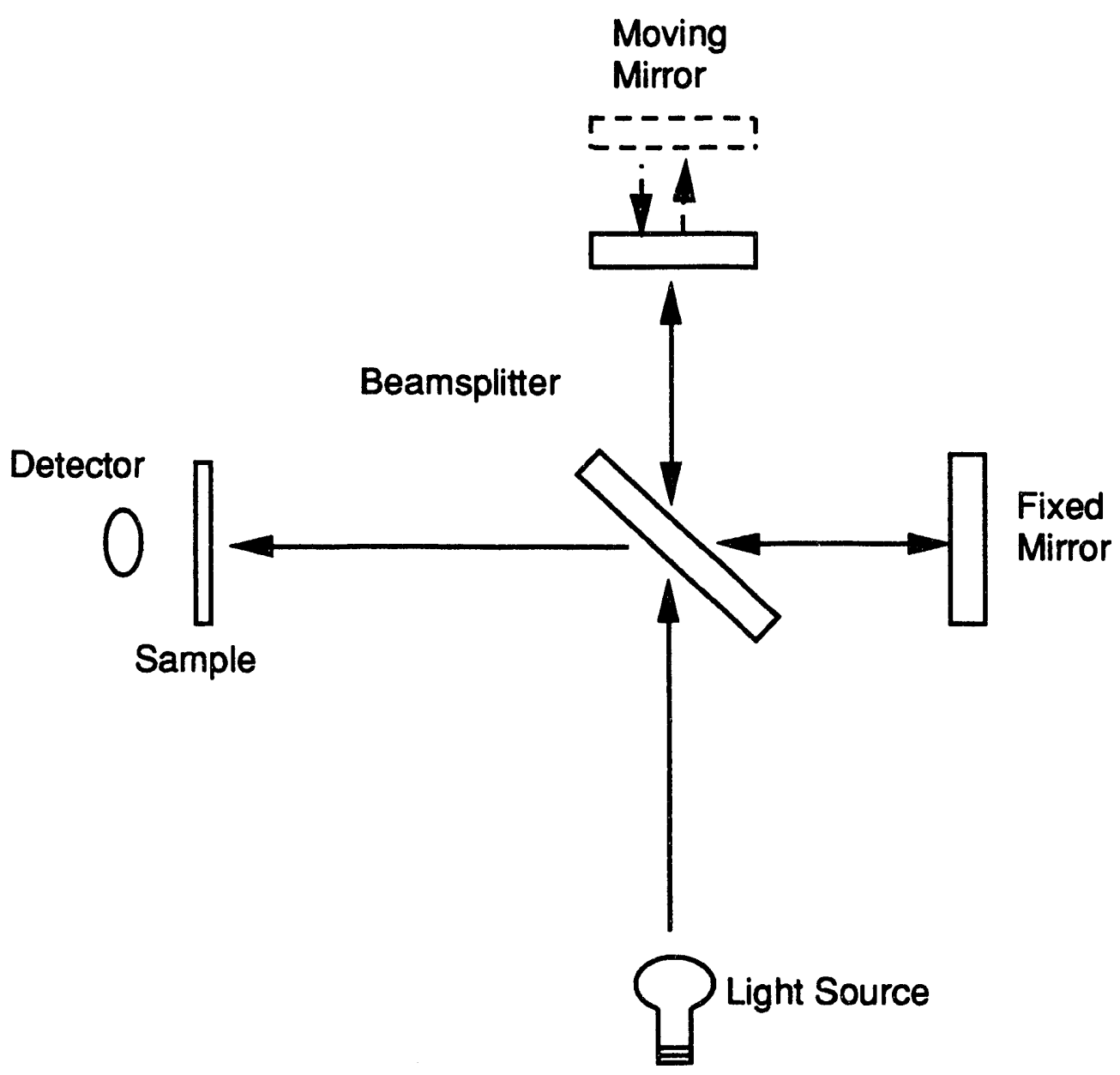

Figure 3.1. - The essential components of a Michelson Interferometer. 
of intensity versus frequency, which is the Fourier transform of the interferogram. The calculation of the Fourier transform of an interferogram is computer intensive so the use of this technique did not become practical until computers with sufficiently large memory and high speed became available. The process of how spectra are obtained using this type of instrument will now be explained in more detail.

We begin the discussion by considering a very simple experimental arrangement in which the light source is a monochromatic laser of wavelength $\lambda$. When the path lengths from the beamsplitter to the two mirrors in Figure 3.1 are equal, the two beams will constructively interfere and there will be an absolute maximum in the intensity of light detected. As the moving mirror is translated, a phase shift develops between the recombining beams which reduces the intensity of the light reaching the detector. When the moving mirror has moved by the distance $\lambda / 4$, the path difference $\Delta$ between the two beams is $\lambda / 2$ and the two beams will destructively interfere, resulting in a minimum of detected light. The sum of the two beams can be shown to be $I_{0}(1+\cos \delta)$, where $I_{0}$ is the intensity at $\Delta=0$ and $\delta=2 \pi(\Delta) / \lambda$. Therefore, the interferogram is simply a cosine wave of wavenumber $k=1 / \lambda$. The Fourier transform of a cosine wave is a delta function at $k=1 / \lambda$, and the spectrum will thus show a single peak at the laser frequency. Because the moving mirror has a limited path length, however, the peak will not be a delta function. The interferogram shows a finite number of sinusoidal oscillations and not an infinitely long wave. A detailed calculation (Bell, 1972) shows that the spectrum of a monochromatic source of wavenumber $k$ ' produced by transforming a finite interferogram is

$$
B(k)=2 L(\sin z / z),
$$


where $L$ is the maximum optical path difference between the two arms of the spectrometer and $z=(2 \pi)\left(k^{\prime}-k\right) L$. This function is referred to as the instrumental line shape. It produces a peak with full width at half maximum of $0.61 / L$. One normally takes enough data points so that the instrumental resolution is higher than the natural linewidths of the peaks being observed.

In an actual spectrometer a broadband source is used and the response at the detector is a sum of cosine waves from all of the different wavelengths present. There is a strong maximum at $\Delta=0$, which is referred to as zero path, since all wavelengths constructively interfere at this point. Far away from zero path the intensity falls to $I_{z e r o}$ path $/ 2$ since the phase of all the different waves is random and

$$
\frac{1}{2 \pi} \int_{0}^{2 \pi} d \theta \cos ^{2} \theta=\frac{1}{2}
$$

An example of an interferogram is shown in Figure 3.2a. The spectrum of the sample, which is the Fourier transform of the interferogram, is seen in Figure 3.2b. The Fourier transform is essentially a calculation of the relative amplitudes of each frequency present in the interferogram. The result is a plot of detected intensity versus $k$.

A critical aspect of spectroscopy is the need for filters to eliminate aliasing, which is the appearance of high frequency spectral features in a low frequency spectrum. To observe radiation of a wavelength $\lambda$ in Fourier transform spectroscopy, one must take a data point every $\lambda / 4$ units of mirror travel, which corresponds to $\Delta=\lambda / 2$, since this is the minimum number of points which will define a sine wave of a given wavelength. This implies that twice as many data points must be taken to observe frequencies up to $4000 \mathrm{~cm}^{-1}$ than to 


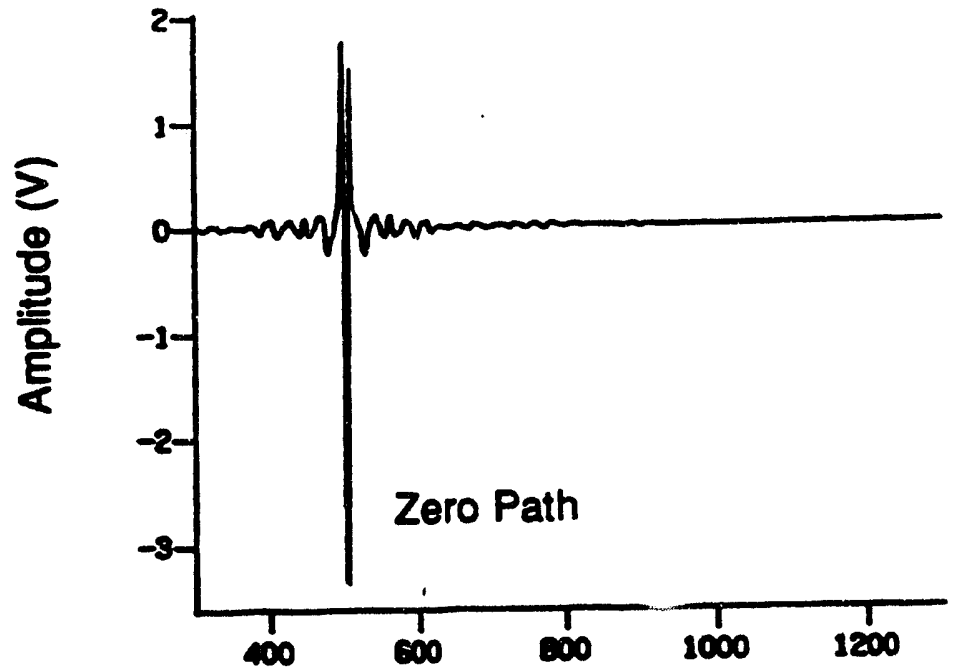

Distance (arb. units)

(a)

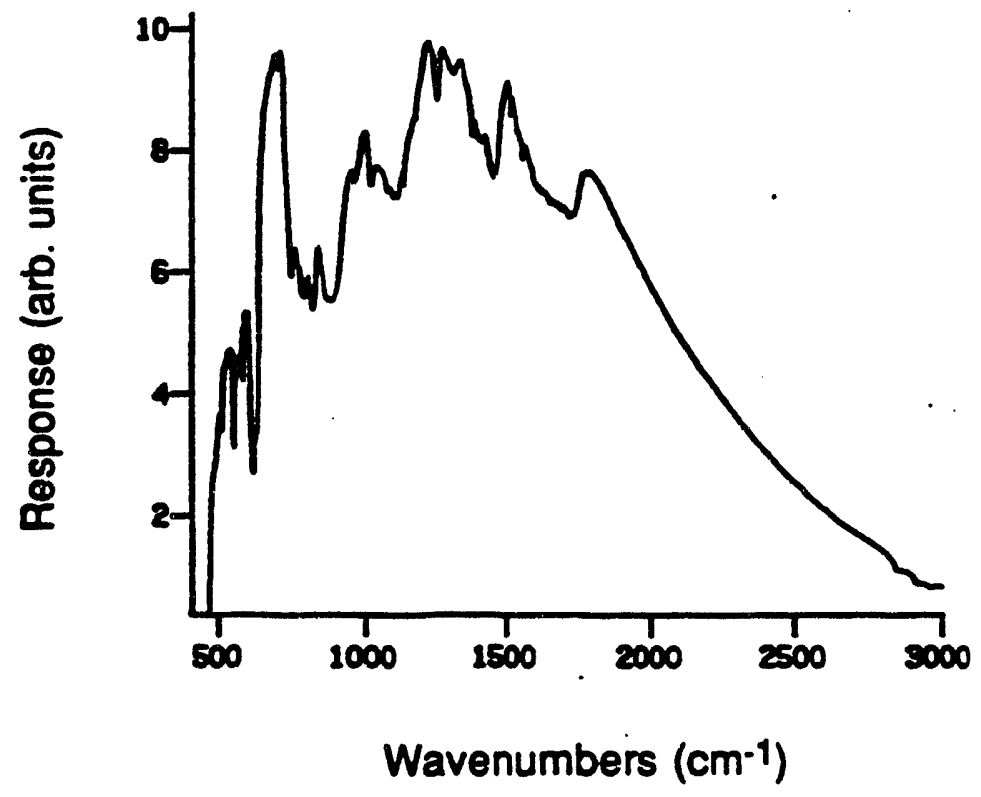

(b)

Fig. 3.2 a) Sample interferogram and b) corresponding spectrum. The response at zero path is negative due to the manner in which the signal is processed (Heyman, 1992). 
observe frequencies up to $2000 \mathrm{~cm}^{-1}$. However, since collection time and computer storage space are proportional to the number of data points recorded, it is desirable to take data only up to frequencies of interest. Aliasing arises when the data are not taken at sufficiently small mirror displacement intervals to observe high frequency light reaching the detector. For example, consider taking data over the range $0-2000 \mathrm{~cm}^{-1}$, which corresponds to taking a point every $1.25 \mu \mathrm{m}$ of mirror travel. If there is light reaching the detector which is of frequency $3000 \mathrm{~cm}^{-1}$ this peak will be aliased into the spectra and will show up at $1000 \mathrm{~cm}^{-1}$. This occurs because 3000 is a multiple of 1000 and the amplitude of the $3000 \mathrm{~cm}^{-1}$ wave will make a contribution to each data point which defines the $1000 \mathrm{~cm}^{-1}$ wave. The way to avoid aliasing in this case would be to either take data up to $3000 \mathrm{~cm}^{-1}$ or filter the incoming light to block radiation above $2000 \mathrm{~cm}^{-1}$. It is much more advantageous to use a filter, as was stated above.

All spectra shown in this thesis were taken over the range $10-1950 \mathrm{~cm}^{-1}$. Two filters were used to block out any higher energy light. First, a mylar beamsplitter was used, and it passes very little radiation above $2000 \mathrm{~cm}^{-1}$. Second, black polyethylene was used. This material is a low pass filter which gently rolls off at higher frequencies. Virtually no light above $2000 \mathrm{~cm}^{-1}$ reaches the detector through this filter when a piece several thousandths of an inch thick is used.

The type of Fourier transform spectroscopy performed in this research is referred to as absorption spectroscopy. In this method, a sample spectrum is divided by a reference spectrum. The reference material is usually identical to the sample material except for the particular aspect under study. For instance, when studying Si impurities in GaAs, the reference material would be GaAs with no Si present. Figures 3.3a and 3.3b shows the spectra obtained for GaAs:Si and an undoped GaAs reference sample. The spectra are then divided and the 


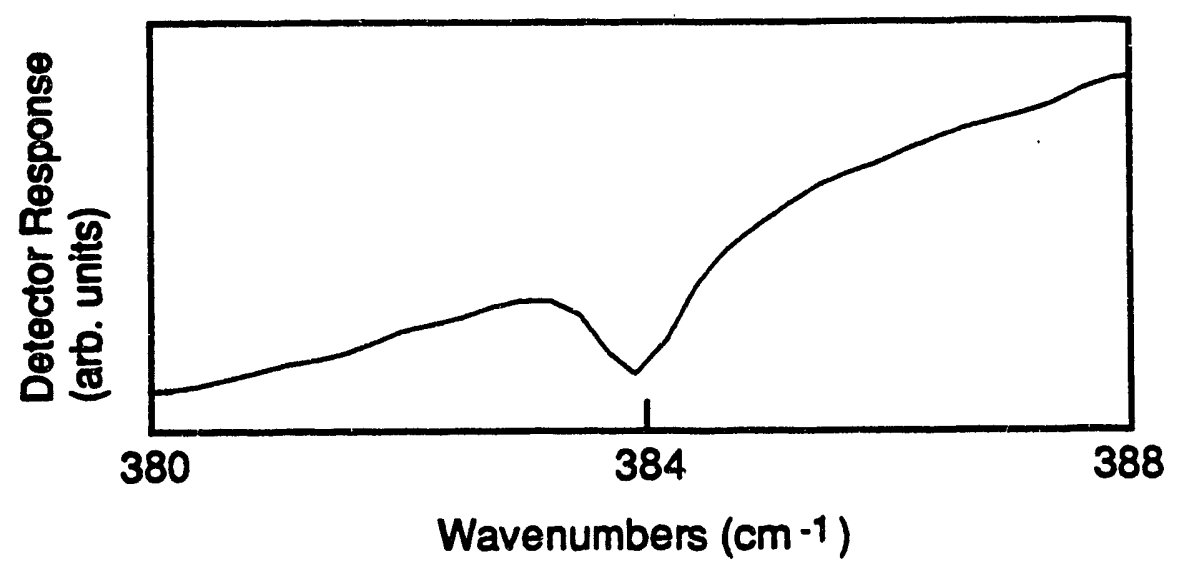

(a)

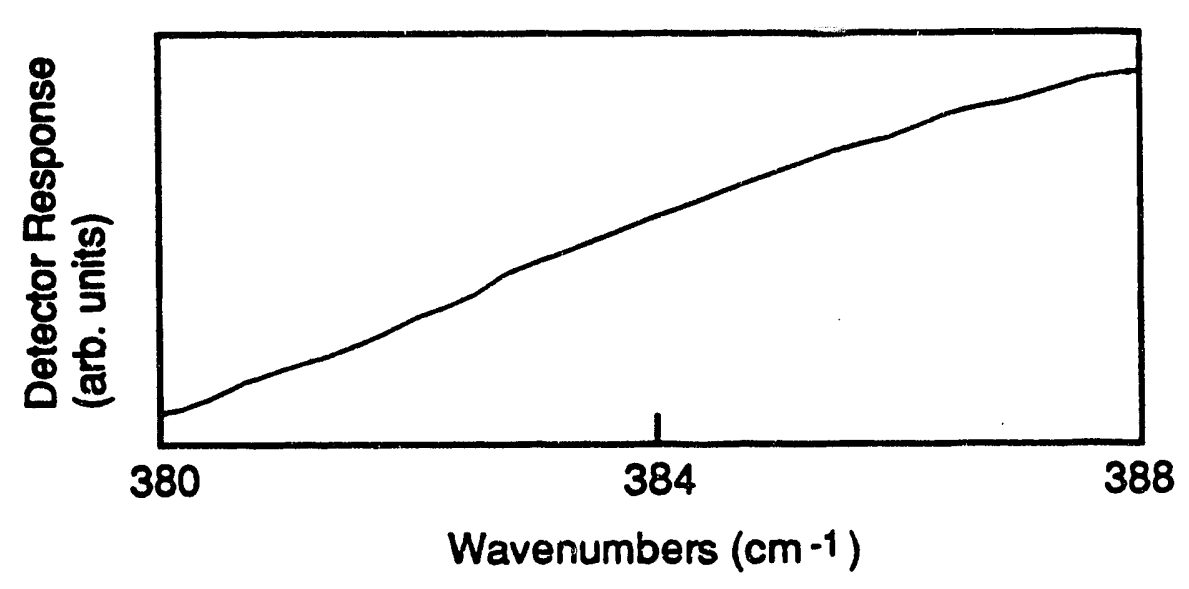

(b)

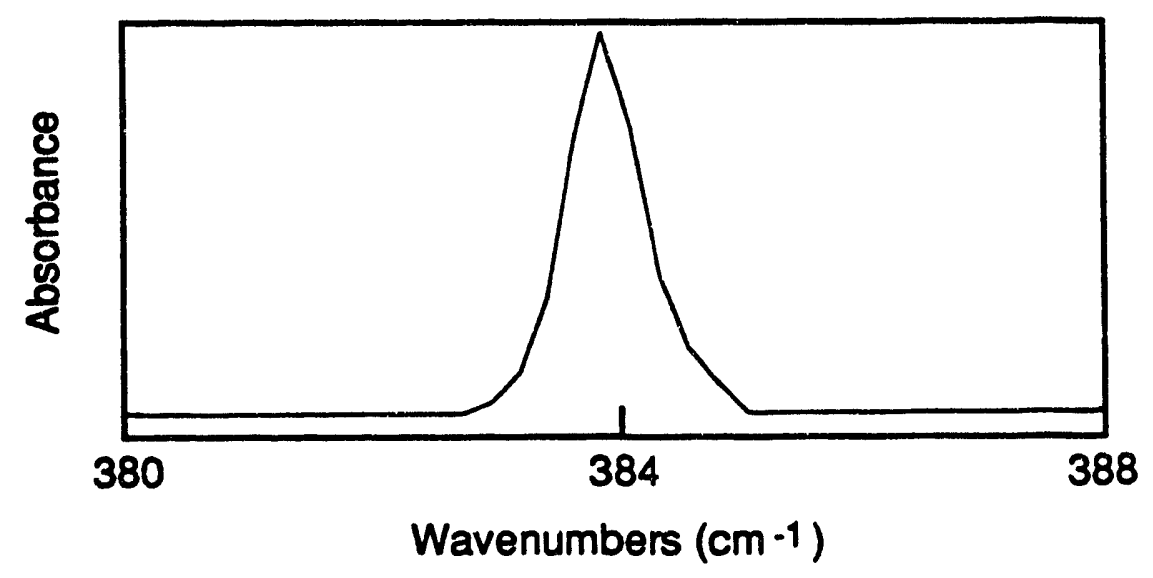

(c)

Fig 3.3. Spectra of a) GaAs:Si sample and b) undoped GaAs reference sample. The absorption spectrum of the GaAs:Si sample, which is the negative logarithm of the ratio of the spectra in (a) and (b), is shown in (c). 
negative logarithm of the result is taken to produce an absorption spectrum, as shown in Figure3.3c. The reason for this procedure is made clear by the following argument. If at a particular frequency Io $_{0}$ represents the intensity of light which reaches the detector through the reference material, then $I_{0} e^{-\alpha x}$ represents the amount of light reaching the detector through the sample, where $x=$ the thickness of the sample and $\alpha=$ the absorption coefficient $=n \sigma$, where $n$ $=$ the concentration of the absorbing defect and $\sigma=$ the defect absorption cross section. Therefore, the process described above for producing an absorption spectrum gives

$$
-\ln \frac{\log ^{-\alpha x}}{10}=\alpha x,
$$

where $\alpha x$ is referred to as absorbance. The final result is therefore a plot of the absorbance vs. $k$ for the impurity under study. As shown in Figure 3.3c, this method yields peaks which are specific to the doped sample and not the reference sample.

The particular spectrometer used for this thesis research is a Digilab 80V spectrometer. A schematic is shown in Figure 3.4. The entire chamber of the spectrometer is evacuated since water vapor in the air has a great many absorption lines in the far-infrared. Since the moving mirror moves on an air bearing, the air fed into this bearing is first run through an air dryer. The source is a SiC globar which is simply a blackbody source run at $1200^{\circ} \mathrm{C}$. While this is not a particularly bright source of infrared radiation, it is the best currently available. Below $100 \mathrm{~cm}^{-1}$ mercury arc lamps are superior light sources. Looking at the figure, one can trace the path of the light from the source to a 


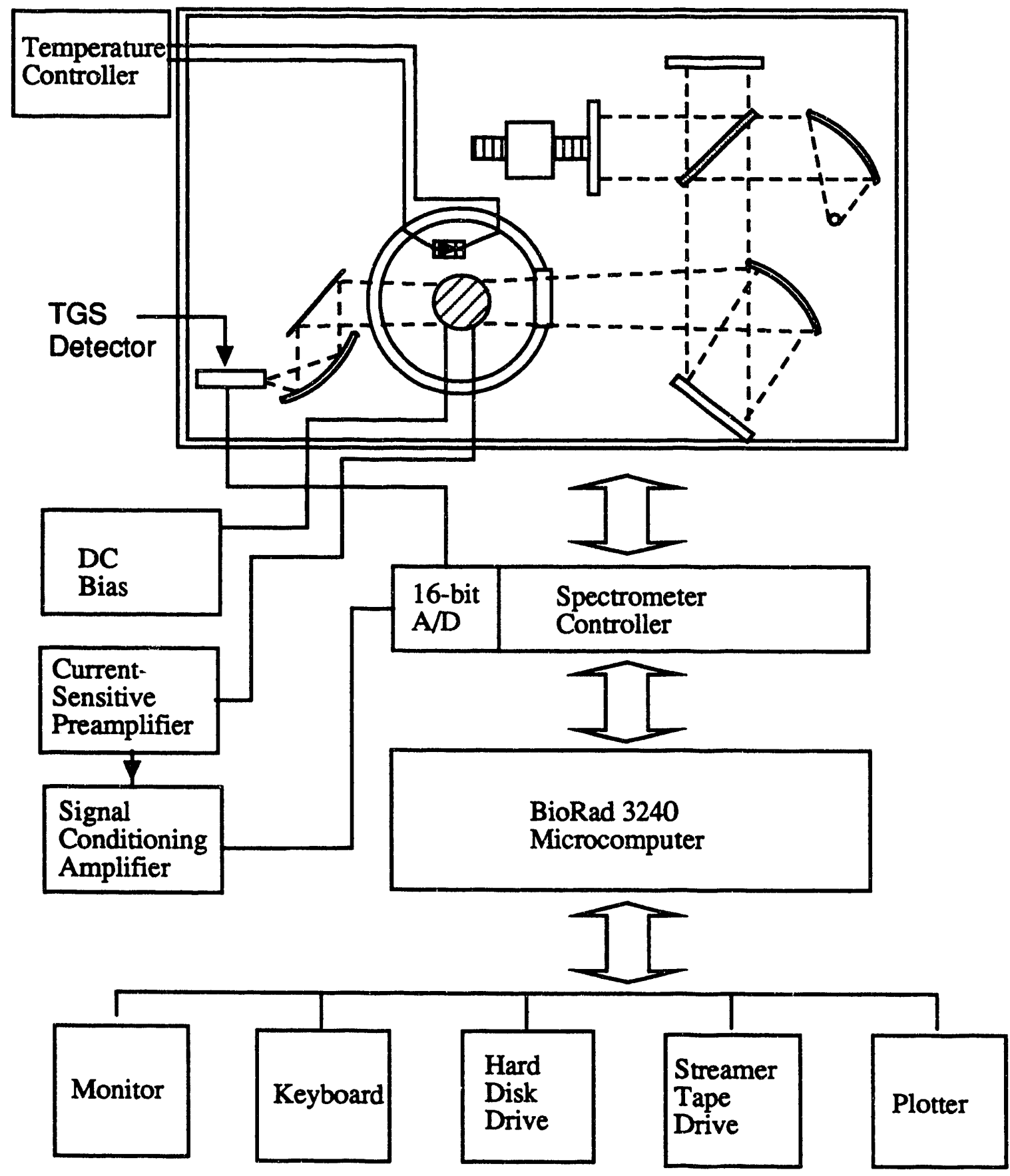

Fig. 3.4. Schematic of Digilag 80-V vacuum Fourier transform spectrometer. Data is taken from the TGS detector or a detector mounted behind the sample, amplified, and then recorded by the computer (Heyman, 1992). 
parabolic mirror which converts the light diverging from the source into a parallel beam.

The light then travels through the interferometer, which is actually composed of two interconnected interferometers. A mirror is mounted on the back of the moving mirror, and this mirror is the moving mirror for a secondary interferometer. This interferometer serves to define the position of the moving mirror in the primary interferometer. There are two additional light sources present for this purpose, a white light source (which is merely a light bulb) and a red $\mathrm{HeNe}$ laser. Since the white light source gives off a large amount of visible radiation, which has a short wavelength in comparison with infrared radiation, the interferogram it produces is very sharply peaked at zero path. This interferogram therefore provides a very accurate zero path position for the primary interferometer. The relative position of the mirror is tracked by counting maxima detected in the interferogram of the $\mathrm{He} \mathrm{Ne}$ laser. As was previously discussed, a maximum occurs each time the mirror moves a distance $\lambda_{\mathrm{He}} \mathrm{Ne} / 2$.

After emerging from the interferometer, the recombined beam is directed by a planar mirror and a weakly focussing parabolic mirror into the sample space. The size of the beam at the focus is roughly $1 \mathrm{~cm}$, and this is where the sample is placed. The detector is usually mounted either directly behind the sample or at a more remote location. The Digilab spectrometer is equipped with a TGS (Tryglycine Sulfate) room temperature pyroelectric detector. An off-axis parabolic mirror is combined with a planar mirror to bring the radiation leaving the sample space to a focus at the detector. A change in temperature of the TGS detector creates a change in its capacitance, and this change is converted into a voltage which is then recorded. The photoconductor detectors used in these studies, which will be described in more detail in Section 3.1.4, detect a photon when it ionizes an impurity electronic level. A bias is kept across the detector 
which sweeps out carriers as they are created. Photoconductive detectors are much more sensitive then pyroelectric detectors, but they do have several disadvantages. First, they cannot detect any radiation below the ionization of the impurity level in the detector. Also, they do not have a flat response as a function of wavelength. Pyroelectric detectors have a response which, although it is not flat, varies linearly with frequency. Despite these disadvantages, photoconductors must be used when photon fluxes are low.

\subsubsection{Grating Spectroscopy}

Another important type of optical spectroscopy is grating spectroscopy. This is very different from Fourier spectroscopy in that spectra are recorded a single wavelength at a time. The heart of a grating spectrometer is a monochromator, which is normally comprised of an entrance slit, mirrors, a grating, and an exit slit. A schematic diagram of a monochromator is shown in Figure 3.5. As shown in the figure, light enters the monochromator through a narrow entrance slit. The light is then made parallel by a parabolic mirror and reflected by a planar mirror onto the grating. The grating of the monochromator is comprised of a series of closely spaced parallel grooves as shown in Figure 3.6. Each groove acts as a radiation source, diffracting the incoming light at various angles. For a given reflection angle $r$, there is a maximum in the intensity of a wavelength $\lambda$ reflected from the grating when the path difference between light reflecting off successive slits is an integral number of wavelengths. This is just the condition for constructive interference and is given by

$$
m \lambda=d(\sin i+\sin r)
$$




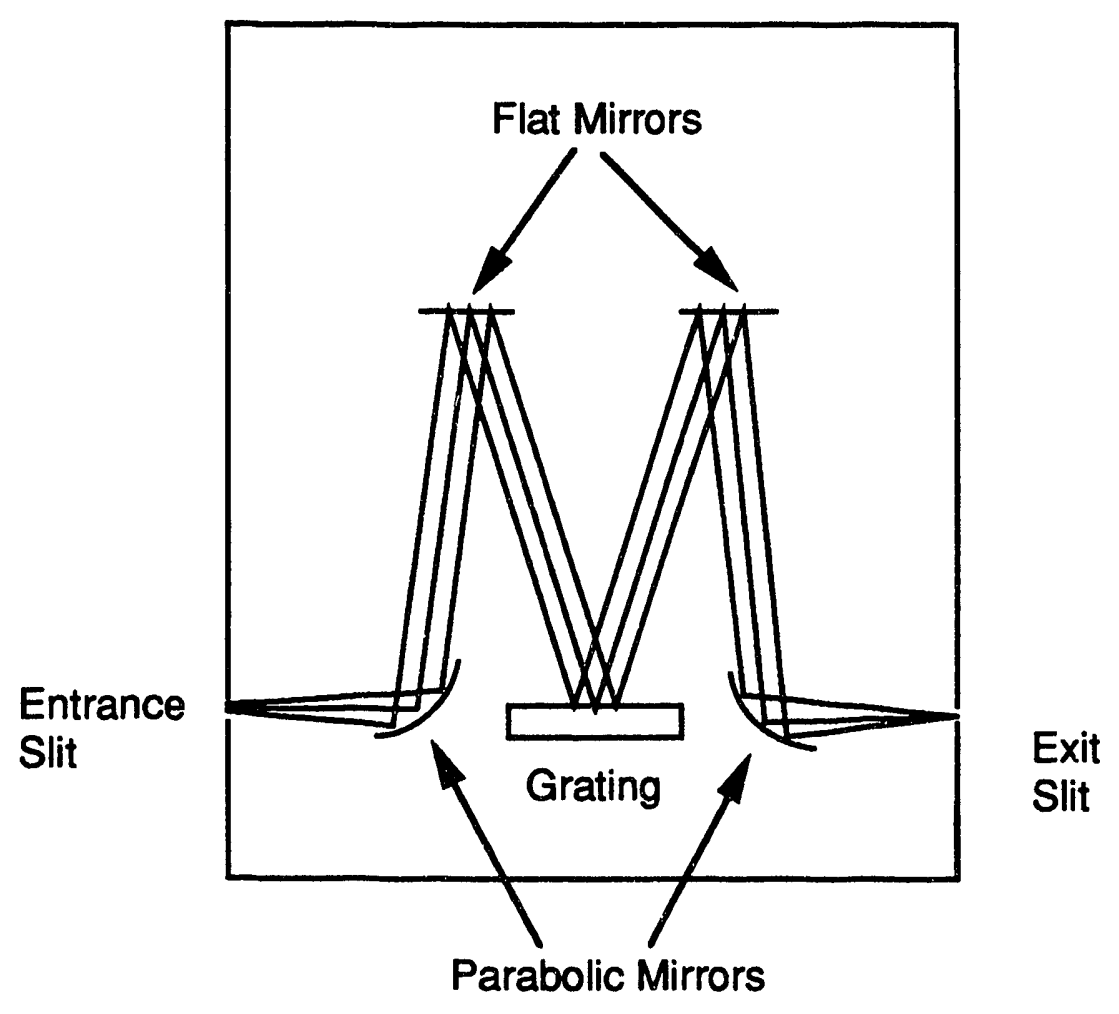

Fig. 3.5. Essential components of a grating spectrometer. 


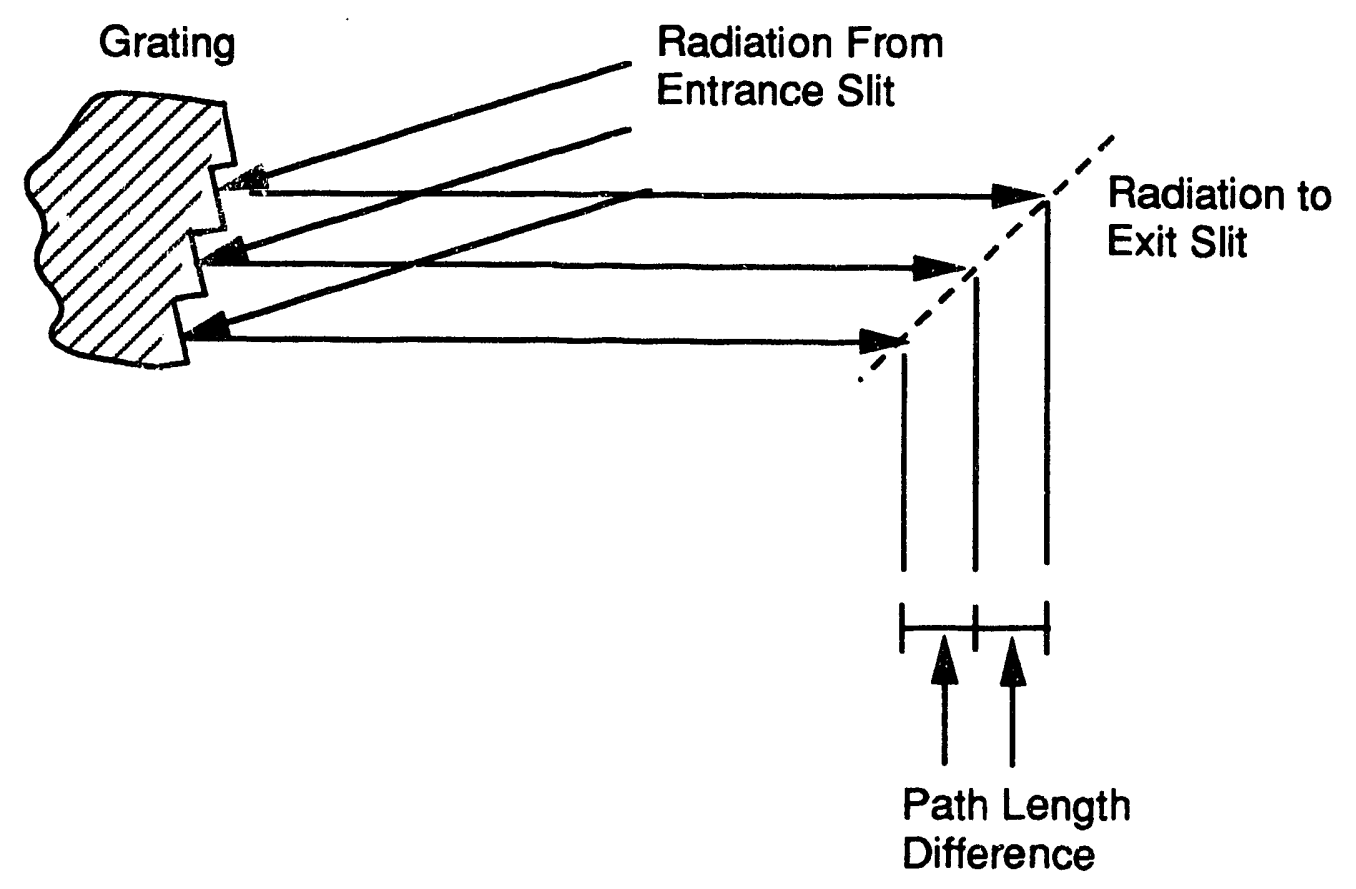

Fig. 3.6. A diffraction grating. When the path length difference is equal to a given wavelength it will constructively interfere (Colthup, 1990). 
where $m$ is the order of the maximum, $d$ is the spacing between the slits, and $i$ and $r$ are the angles of incidence and reflection measured from the normal to the grating surface. First order implies that there is a path difference of one wavelength between adjoining slits, and so on for higher orders. The light is then reflected by mirrors through the exit slit. If light of wavelength $\lambda$ passes through the exit slit of the monochromator, this implies that higher orders of radiation with wavelengths $\lambda / 2, \lambda / 3$, etc. will also exit through the slit. This radiation must be eliminated using appropriate filters if this is important. By rotating the grating one can choose the wavelength of light which travels through the monochromator and reaches the sample. A spectrum is produced by rotating the grating through a given angular range, recording the response at each wavelength. The resolution is limited by the width of the entrance and exit slits and the number of grooves per $\mathrm{cm}$ on the grating.

A grating spectrometer takes the light from the exit of a monochromator and sends it through the sample to a detector. The grating spectrometer used in these studies is a Cary 2390 spectrophotometer. A schematic of the apparatus is shown in Figure 3.7. An extremely useful feature of this equipment is that the source beam is chopped. This is important because it implies that the spectrometer can be used in conjunction with a lock-in amplifier to detect weak signals. It is capable of spectroscopy from the near infrared all the way to the ultraviolet region of the spectrum, though different sources, gratings, and detectors are used for different wavelength regions. Ultraviolet and visible lamps are used as sources in the appropriate ranges. Different gratings are used below and above $800 \mathrm{~nm}$ because it is only possible to optimize grating geometries for a particular wavelength, and the grating efficiency decreases rapidly away from this optimum wavelength. A photomultiplier tube is used to detect visible radiation while a PbS detector is used to detect near infrared 
Monochromator

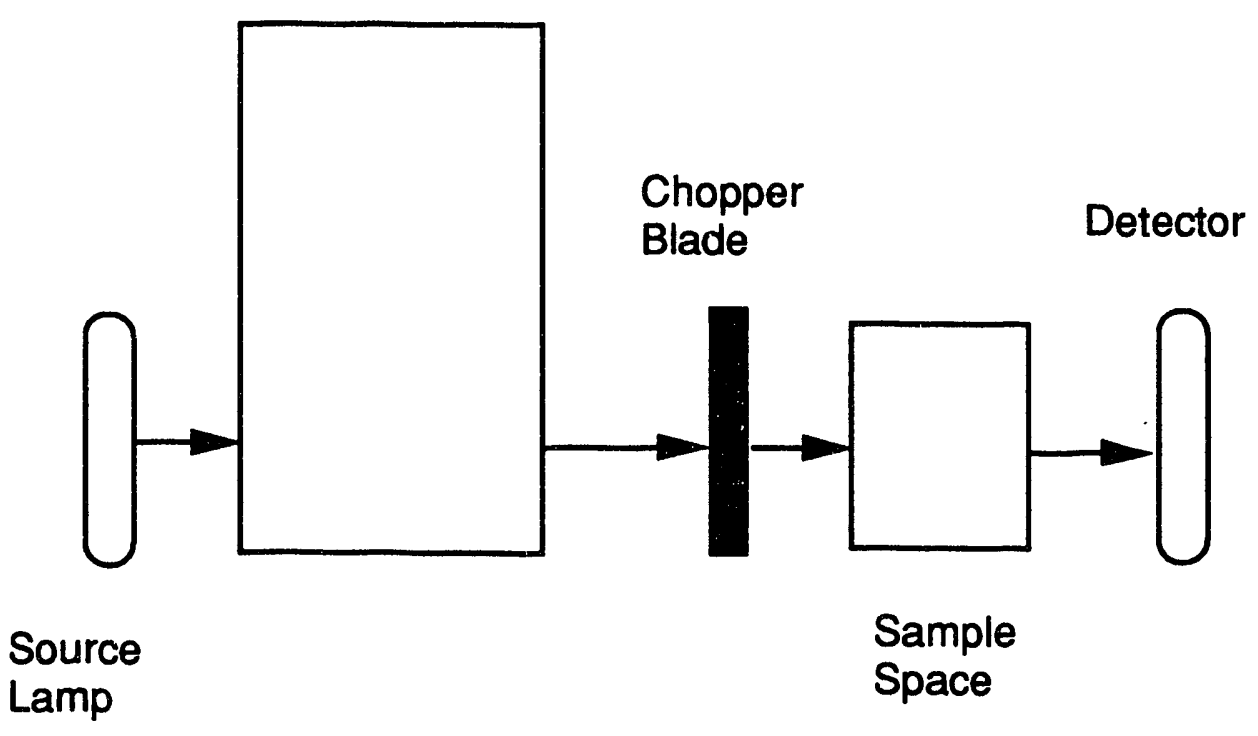

Fig. 3.7. Schematic of the Cary 2390 grating spectrometer. The arrow shows the path of the light beam. For the work described in the this thesis, the built-in detectors were not used. 
radiation. The $\mathrm{PbS}$ detector is a photoconductor which detects excitations from the valence band to the conduction band. Although the Cary spectrometer is a high performance instrument, for the work done in this thesis it was used only as a source of monochromatic light.

\subsubsection{Comparison of Fourier Transform and Grating Spectroscopy}

There are several advantages of a Fourier transform spectrometer over a grating spectrometer, and this explains why the bulk of the optical work in this thesis was performed on a Fourier transform machine. The main advantages are normally referred to as the Fellgett (Fellgett, 1958) and Jacquinot advantage (Jacquinot, 1960). The most important conclusion which follows from the Fellgett advantage is that it is possible to acquire comparable spectra in less time using a Fourier transform spectrometer than a grating spectrometer. This is a consequence of the fact that each scan of an interferometer (which is defined as one translation of the moving mirror along its full length of travel) contains information about the whole range of wavelengths being observed while a data point in a grating spectrum only contains information about a single wavelength. The Fellgett advantage can be considered quantitatively as follows. If one is measuring a spectrum using a monochromator over a range of wavenumbers $k_{1}-k_{2}$ with resolution $\Delta k$, then the number of points in the spectrum is

$$
M=\left(k_{1}-k_{2}\right) / \Delta k .
$$

If the time for the total spectrum to be observed is $T$, then each point in the spectrum will take time (T/M) to observe. Assuming the noise is independent of 
the signal intensity, then the signal-to-noise ratio (SNR) for a grating spectrum is given by

$$
\text { SNR grating } \alpha(T / M)^{1 / 2} \text {. }
$$

Since in an interferometer all wavelengths are observed simultaneously, the signal to noise ratio is proportional to

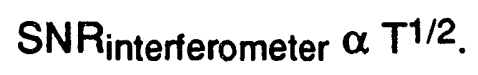

Therefore, the advantage of the interferometer over the grating spectrometer is proportional to $M^{1 / 2}$. Since $M$ can easily be on the on the order of 10,000 , this represents a substantial advantage. It should be pointed out that in the visible range where excellent detectors are available and the noise is photon limited, i.e. it is proportional to the square root of the signal, then the Fellgett advantage disappears. This advantage is also irrelevant if only a small spectral range is being observed.

The Jacquinot advantage of interferometers over grating spectrometers is a function of the relative optical throughput of these instruments. The resolution of a grating spectrometer depends linearly on the entrance and exit slit width. In contrast, the resolution of an interferometer is dependent for the most part on the length which the moving mirror travels. This implies that the optics at the entrance of an interferometer can be made large without having any significant reduction in the resolution of the instrument. Thus, for a given resolution, the slit sizes for the grating spectrometer are never comparable to the source and detector areas possible with an interferometer. High resolution in a grating spectrometer also requires large focal radii for the collimating mirrors, which 
results in small solid angles. Again, this is not a problem for an interferometer where the light is not being dispersed. For these reasons, the interferometer has a throughput roughly 200 times that of the best grating instruments and this results in a significant decrease in the amount of time required to obtain a spectra of a given SNR.

Despite the advantages of an interferometer over a grating spectrometer, there is a very important circumstance where the use of grating type instrument is crucial. For the study of some semiconductor defects, it is sometimes important to shine only a single wavelength at a time on the sample. This is the case for DX centers since certain wavelengths of light can convert them into shallow donors.

\subsubsection{Optical Spectroscopy in the Diamond Anvil Cell}

Fourier transform and grating spectroscopy of semiconductor defects in a diamond anvil cell (DAC) form the basis of the research described in this thesis. Prior to this work, though, the technique for performing this type of spectroscopy had not been developed. It is easy to understand the difficulties associated with performing spectroscopy of samples mounted in a DAC. The sample size is limited to the order of a few hundred micrometers in diameter and roughly 100 $\mu \mathrm{m}$ in thickness. While it is common practice to do spectroscopy in a DAC on samples where the absorption is due to intrinsic effects (i.e., $10^{22}$ atcms $/ \mathrm{cm}^{3}$ ), spectroscopy of defects, where typical concentrations are $1017-10^{18} \mathrm{~cm}^{-3}$, requires greatly enhanced sensitivity. The problem is one of achieving sufficient signal-to-noise ratio, and the remainder of this section will describe the experimental set-up used to overcome this problem. 
The assembly shown in Figure 3.8 is the most important component for the experimental work described in this thesis (Wolk, 1991a). It is a monolithic assembly consisting of a mirror, light concentrating cone, diamond anvil cell, and photoconductor detector. It is mounted vertically into a liquid helium cryostat placed at the sample focus of either the Fourier transform or the grating spectrometer used in this work. The mirror directs the light which is travelling horizontally through the spectrometer upwards towards the sample. The mirror currently in use is a flat mirror, but a parabolic mirror would be superior in that it would help focus the light onto the sample. The cone serves to concentrate the spectrometer light on the sample. It is a straight cone, which should be replaced by a Winston cone in order to optimize the system. A straight cone is roughly $70 \%$ as efficient as a Winston cone. The only reason these suggested improvements were not tried initially is that they are much more difficult and costly to fabricate.

A photoconductor detector is mounted directly behind the cell. Both $\mathrm{Ge}: \mathrm{Be}$ and $\mathrm{Ge}: \mathrm{Cu}$ photoconductors, which have photoconductive onsets at 200 $\mathrm{cm}^{-1}$ and $350 \mathrm{~cm}^{-1}$, respectively, were used for these studies. These detectors are most sensitive for low photon fluxes, and the responsivity and noise equivalent power (NEP) of the Ge:Be detector are shown as a function of bias for a particular set of operating conditions in Figure 3.9 (Haegel, 1983). From the figure it is clear that for these conditions the detector has a high responsivity and an NEP which is not much larger than the photon limited background NEP, which is roughly $10^{-16} \mathrm{~W} \sqrt{\mathrm{Hz}}$. For this experiment, we are in a regime of higher photon flux than the case shown in Fig. 3.9, but the detector is still performing close to the photon noise limit. The detector was designed to fill the cavity it is mounted in, and the cavity is spherical in the back to increase light collection efficiency. 


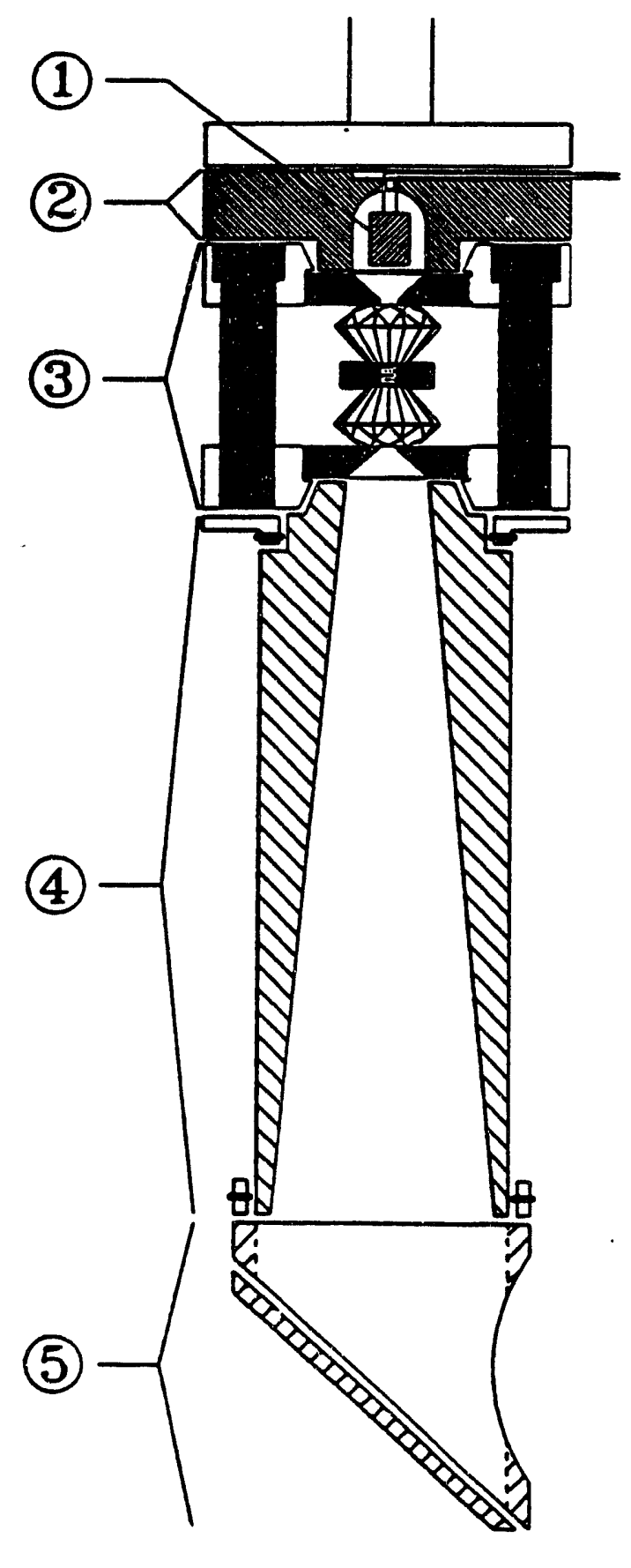

XES 922-288

Fig. 3.8. Schematic of monolithic assembly used to perform spectroscopy of samples mounted in a diamond anvil cell. 1) photoconductor 2) photoconductor mount 3) diamond anvil cell 4) light concentrating cone 5) mirror assembly. 


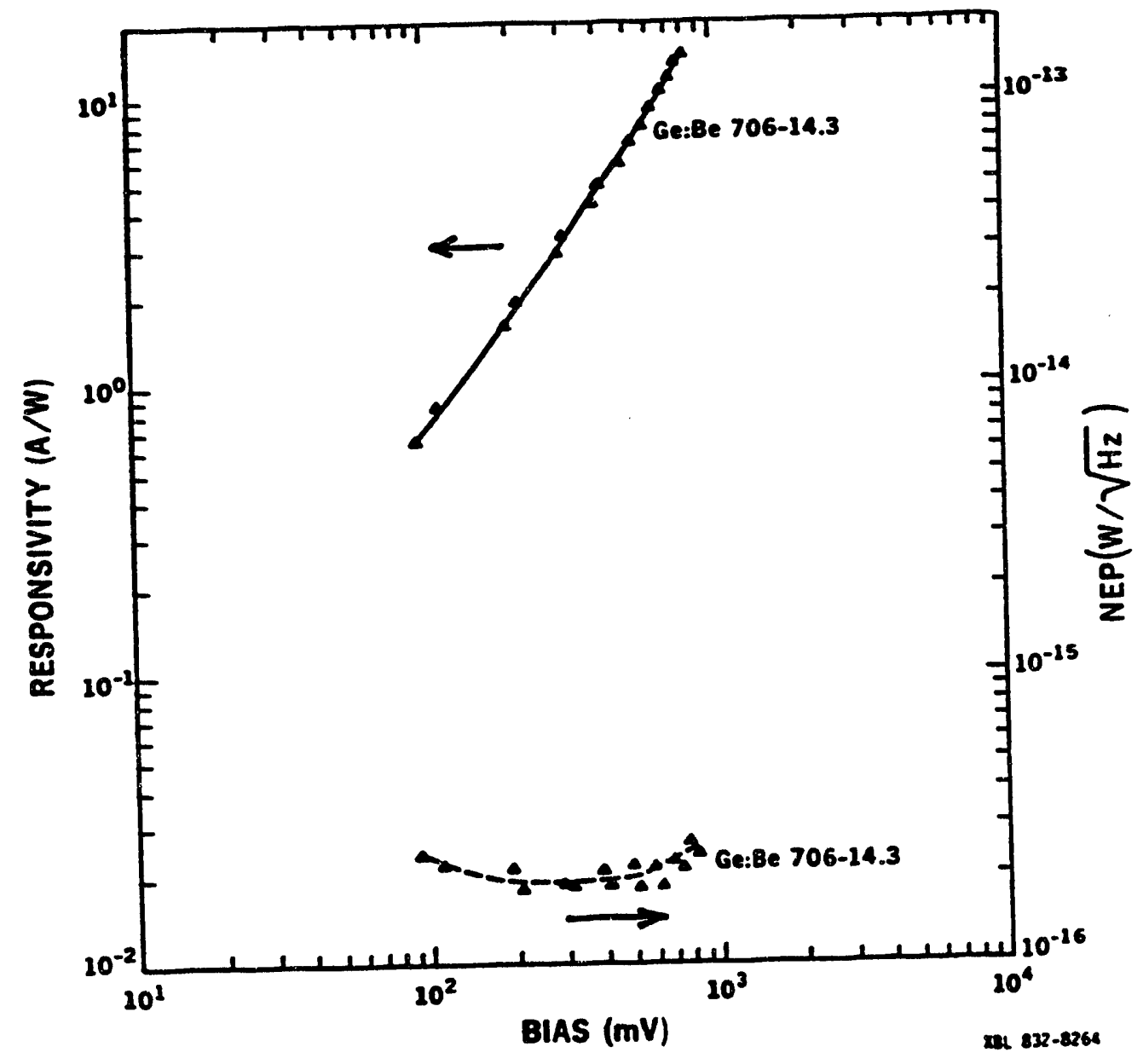

Fig. 3.9. Responsivity and noise equivalent power (NEP) of the Ge:Be phtoconductor used for the work in this thesis. Condtions for this data are $v=238 \mathrm{~cm}^{-1}$, photon rate $=1.5 \times 10^{8}$ photons $/ \mathrm{s}$, and $T=4.2 \mathrm{~K}$. The photon NEP for the test conditions is $\approx 10^{-16} \mathrm{~W} / \sqrt{\mathrm{Hz}}$. (Haegel, 1983). 
This design has several critical features. First and most importantly, the detector is mounted directly behind the cell, insuring that a significant fraction of the photons which make it through the DAC will be collected by the detector. Second, the design virtually eliminates stray radiation, a small amount of which could dominate the small number of photons reaching the detector through the DAC. Finally, alignment is trivial since it is only requires the line-up of the 10 $\mathrm{mm}$ beam with the $12.7 \mathrm{~mm}$ diameter entrance to the mirror assembly. If the detector is separated from the DAC, which is the set-up commonly used by other groups performing Fourier transform spectroscopy of samples mounted in a DAC, positioning of the cell becomes extremely critical and therefore very difficult.

\subsection{Hall Effect}

The final characterization technique to be discussed is Hall effect analysis, which is used to determine the free carrier concentration in samples. The principle of Hall effect is pictured in Figure 3.10. The figure shows the cross-section of a sample which has wires attached to the top and bottom as well as each side. The sample is in a magnetic field B pointing in the z-direction and a current is passed through the sample in the $x$-direction. The Lorentz force $e(v \times B)$ drives the electrons downward, where $e$ is the charge on the electron and $v$ is the velocity of the electron. This occurs until an electric field $E$ of sufficient strength is built up and creates a force eE that balances the magnetic force. The forces balance when

$$
e E=e(v \times B) .
$$




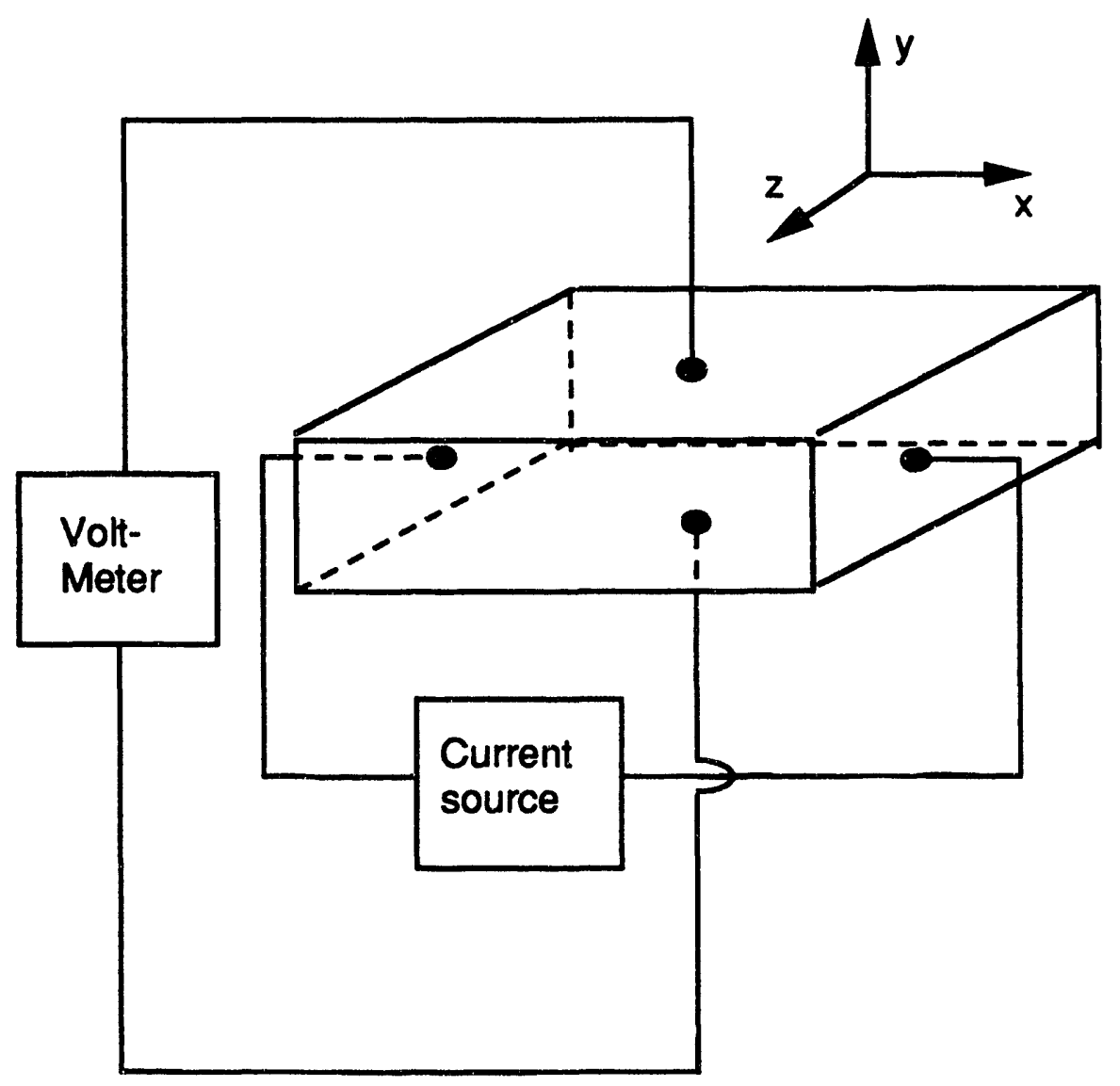

Figure 3.10. Standard Hall effect geometry.

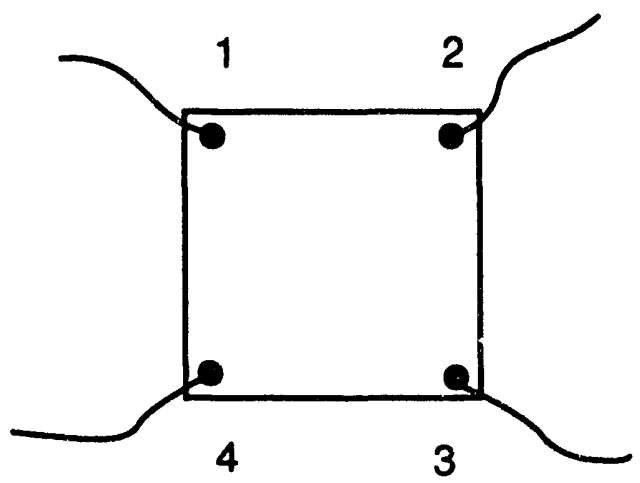

Figure 3.11. Van der Pauw arrangement of contatcs for Hall effect analysis. 
For the geometry shown in Figure 3.10, this reduces to $E_{y}=v B_{z}$. The Hall coefficient $R_{H}$ is defined as

$$
R_{H}=E_{y} /\left(j_{x} B_{z}\right)
$$

Using the relationship $\mathrm{j}=$ nev, where $\mathrm{n}$ is the free carrier concentration, gives

$$
R_{H}=1 /(n e) \text {. }
$$

Since $\mathrm{j}$ and $\mathrm{B}$ are controlled by the experimenter, and a measurement of the voltage across the sample in the $y$-direction provides the value of $E_{y}$, the free carrier concentration can be determined directly.

In most cases, a van der Pauw arrangement of contacts is used. In this arrangement, all four contacts are on the top of the sample as shown in Figure 3.11. The resistivity of the sample can then be shown to be (van der Pauw, 1958)

$$
\rho=\frac{\pi t}{\ln (2)} \frac{R_{12,34}+R_{23,41}}{2} f
$$

where $t=$ the thickness of the sample, $R_{12,34}=$ the resistance obtained form the ratio of the voltage across contacts 2 and 3 and the current through contacts 1 and $4, R_{23,41}$ is similarly described, and the factor $f$ depends on the ratio of the resistances. For a square sample $f=1$. The Hall coefficient in this case is

$$
R_{H}=\left(\Delta R_{24,13}\right) t / B \text {, }
$$


where $\Delta R_{24,13}$ the change in resistivity when the $B$ field is turned on, implying that the free carrier concentration is equal to

$$
n=B /\left(\Delta R_{24,13}\right) e
$$

These data can also be used to calculate the mobility in a sample, which is just the speed of the free carrier per unit electric field. We have

$$
\mu=\frac{v}{E}=\frac{1}{n e \rho}=\frac{2 \ln (2)}{(\pi t)} \frac{\Delta R_{24,13}}{R_{12,34}+R_{23,41}} \frac{1}{f} .
$$

The mobility provides a measure of the number and type of scattering centers present in a semiconductor. The three main types of scattering mechanisms are ionized impurity scattering, which is essentially an example of Rutherford scattering, neutral impurity scattering, and electron-phonon scattering.

Because all of the samples studied in this thesis were heavily doped, ionized impurity scattering was the dominant mechanism limiting mobility. The number of ionized impurities in a sample depends on the number of defects which compensate the majority impurity. If there are $10^{18} \mathrm{~cm}^{-3}$ shallow donors and $1017 \mathrm{~cm}^{-3}$ acceptors, 1017 of the donor electrons will fall into the acceptor states, as shown in Figure 3.12. This is simply a matter of the electrons falling into the lowest energy state which is unoccupied. This implies that there will be $2 \times 10^{17}$ ionized impurities in the sample. The compensation ratio, $\theta$, is defined as

$$
\frac{\text { concentration of minority impurity }}{\text { concentration of majority impurity }}
$$




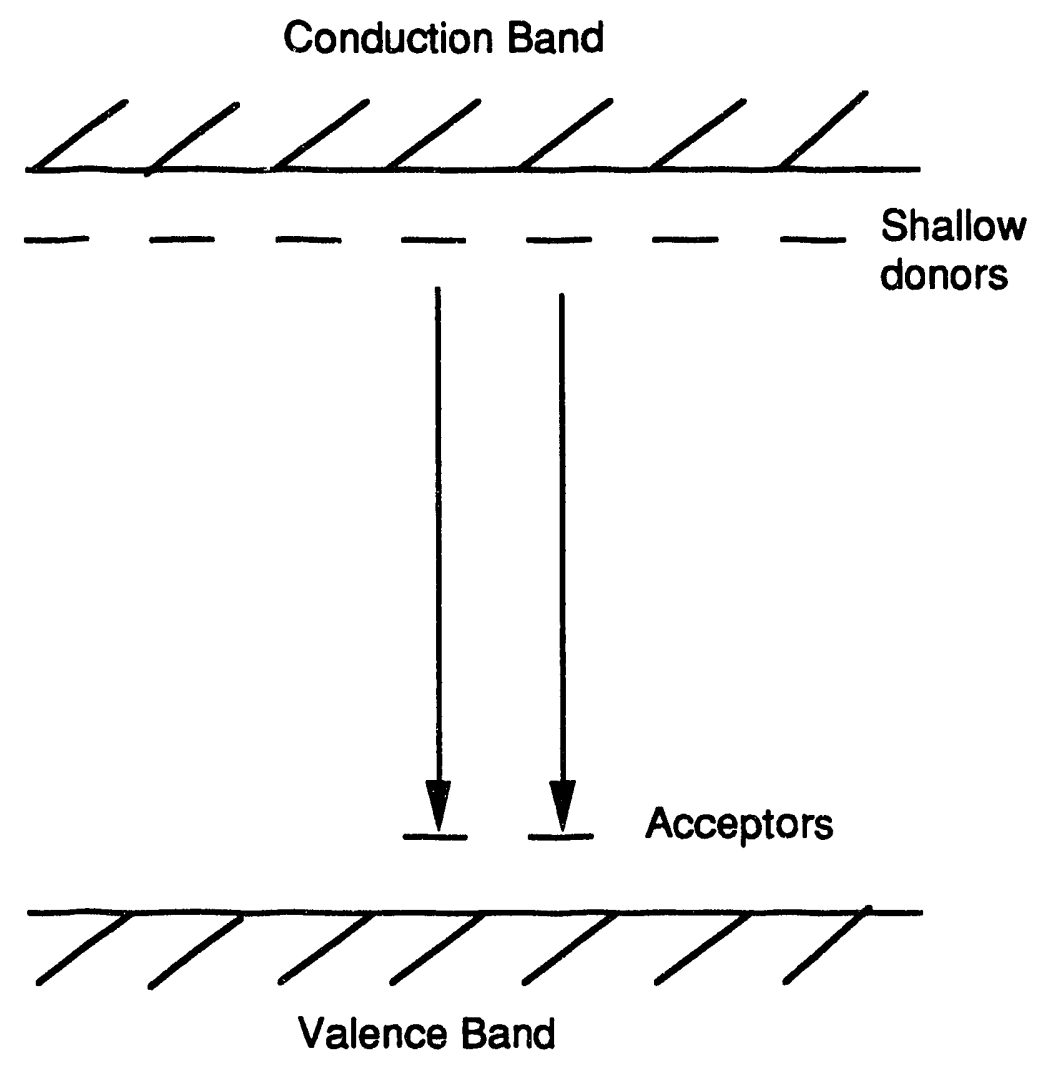

Fig 3.12. Minority impurities, in this case acceptors, compensate the majority donors. All of the acceptor levels and an equal number of donor levels are ionized. 
It is clearly related to the ionized impurity concentration, which in turn determines the mobility in a heavily doped sample. This relationship will be used in the next chapter to determine the compensation ratio of the GaAs samples studied in this thesis. 


\section{LVM Spectroscopy of DX Centers in GaAs:Si}

\subsection{Introduction to Local Vibrational Modes and their Observation}

\subsubsection{Theoretical Description of Local Vibrational Modes}

The experiment described in this chapter uses the observation of local vibrational modes (LVMs) of defects in GaAs:Si to determine the charge state and study the microscopic structure of the Si DX center. It is therefore appropriate to begin with a discussion of how these modes arise and can be observed. An LVM is always associated with the vibrational motion of an impurity atom which is lighter than the atoms comprising the host lattice. Because the impurity is lighter, its vibrational motion does not significantly affect the surrounding portion of the lattice and only the atoms in the first few nearest neighbor shells experience any significant movement from their equilibrium positions.

We begin the discussion of local vibrational modes by considering phonons, which are completely delocalized vibrational modes. We start by considering an $\mathrm{N}$-atom, one-dimensional diatomic chain with lattice spacing a, as shown in Figure 4.1. The masses of the atoms are $M$ (heavier atom) and $m$ (lighter atom), the force constants for the springs are $k$, and only nearest neighbor interactions are considered. Using cyclic boundary conditions and taking $N$ to be even, the equations of motion for the atoms in the chain are given by 


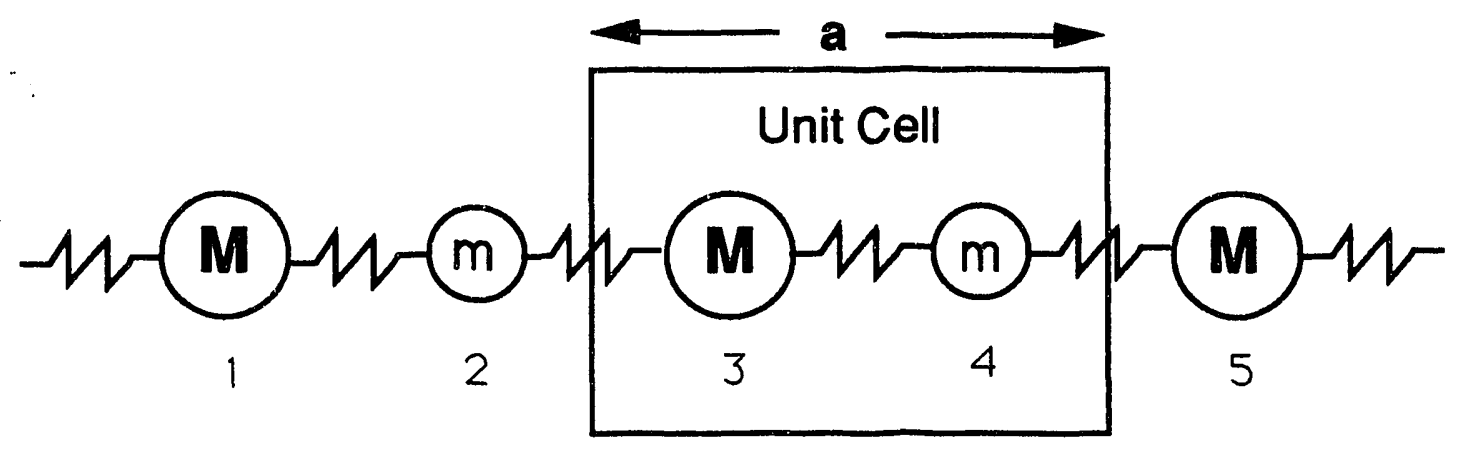

Fig. 4.1. Diatomic linear chain with nearest neighbor force constants (Barker, 1975). 


$$
\begin{gathered}
M \ddot{x}_{1}=k\left(x_{2}-x_{1}\right)+k\left(x_{N}-x_{1}\right) \\
m \ddot{x}_{2}=k\left(x_{3}-x_{2}\right)+k\left(x_{1}-x_{2}\right) \\
M \ddot{x}_{3}=k\left(x_{4}-x_{3}\right)+k\left(x_{2}-x_{3}\right) \\
\cdot \\
M \ddot{x}_{N}=k\left(x_{1}-x_{N}\right)+k\left(x_{N-1}-x_{N}\right) .
\end{gathered}
$$

These equations can be rewritten in matrix form as

$$
M \overrightarrow{\ddot{x}}=k \vec{x} \text {, }
$$

where

$$
\underline{M}=\left(\begin{array}{ccccc}
M & 0 & 0 & & \\
0 & m & 0 & & \ldots \\
0 & 0 & M & & \\
& \vdots & & & \ddots
\end{array}\right)
$$

and

$$
\underline{k}=\left(\begin{array}{cccccc}
2 k & -k & 0 & 0 & . . & -k \\
-k & 2 k & -k & 0 & . . & 0 \\
0 & -k & 2 k & -k & . . & 0 \\
: & : & : & & & \\
0 & 0 & 0 & & \ddots & \\
-k & 0 & 0 & & &
\end{array}\right)
$$

We now look for a solution in the form of a travelling wave

$$
x=e^{i(q n a / 2-\omega t)}
$$


where $q=2 \pi / \lambda$ is the wavevector and $\omega$ is the frequency of the wave. In other words, we are assuming that we can find solutions that vary sinusoidally in both time and space. Substituting this expression into Eq. 4.2 gives

$$
\left(\underline{k}-\omega^{2} \underline{M}\right) \vec{x}=0 .
$$

This equation has solutions only for values of $\omega^{2}$ which make the determinant of the matrix $\left(\underline{k}-\omega^{2} \underline{M}\right)$ equal to zero. The problem therefore reduces to a diagnolization of this matrix where the eigenvalues are the allowed values of $\omega^{2}$ and the eigenvectors give the displacements of each atom.

This equation was solved by Barker and Sievers (1975) for a $2 \mathrm{~N}$ atom chain of GaP ( $M=70 \mathrm{amu}, \mathrm{m}=31 \mathrm{amu}$ ). The resulting dispersion relationship is shown in Figure 4.2 for the case $\mathrm{N} \rightarrow \infty$. There are two main branches of the dispersion relationship, an optical and an acoustic branch, separated by a gap. The optical branch is so named because neighboring atoms move in opposite directions and this motion can thus be excited in an ionic compound such as GaP by an electromagnetic wave. In the acoustic branch neighboring atoms move in the same direction. The $q$ values are limited to the range $-\pi / a \leq q \leq$ $+\pi / a$ since it does not make sense physically to have a wavelength shorter than twice the lattice spacing.

Since it will be shown that the introduction of a defect into the diatomic chain will result in modes which no longer have a sinusoidal spatial dependence, it is useful for the purposes of comparison to describe the perfect chain in a q-independent manner. One method of accomplishing this is to use the density of states $g(\omega)$, where 


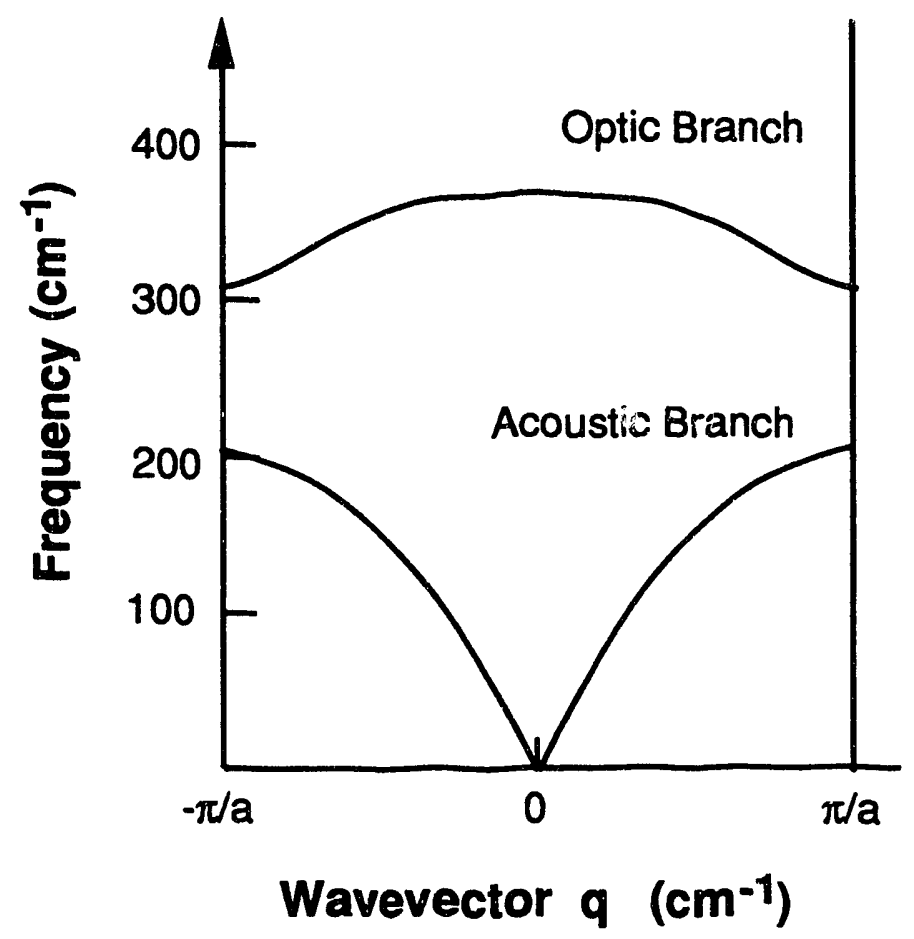

Fig 4.2. Dispersion relationship for diatomic chain with $\mathrm{N}$ atoms of mass $M=70$ amu and $N$ atoms of $m=31$ amu, as $N \rightarrow \infty$. (Barker, 1975). 


$$
N=\int g(\omega) d \omega
$$

and $N$ is the total number of modes. Figure 4.3 shows a calculation of $g(\omega)$ versus $\omega$ for an infinitely long GaP chain.

We now examine the effect of replacing a $P$ atom $(m=31 \mathrm{amu})$ with an atom of $m=20 \mathrm{amu}$, as pictured in Figure 4.4. One difference is that one half of the modes, all those in which the impurity atom undergoes some motion, are affected. This is to be expected since the introduction of the impurity disturbs the periodicity of the chain, leaving a single reflection plane and therefore separating the solutions into those of odd and even parity. If the impurity does not move in a given mode its frequency is unaffected, but modes which do couple to the impurity are shifted to slightly higher frequencies.

The most striking result of this substitution is that a new mode appears which has a frequency of $416 \mathrm{~cm}^{-1}$, well above the highest optical frequency mode $\left(370 \mathrm{~cm}^{-1}\right)$. This is the local vibrational mode, and it can be seen appearing in the plot of $g(\omega)$ in Figlte 4.5. In contrast to the phonon modes, the local mode has a highly non-sinusoidal character and the amplitude of the nearest neighbor vibrations dies off quickly as one moves away form the impurity. This behavior is illustrated in Figure 4.6. A physical argument for the shape of the mode is that a high frequency mode is unable to propagate through the chain since it would be outside i. 1e range of allowed eigenvectors of the perfect chain. Thus, the mode must decay rapidly as one moves away from the impurity site. This manner of thinking is justified mathematically since it is found that the secular equation cannot be solved for the LVM frequency unless 


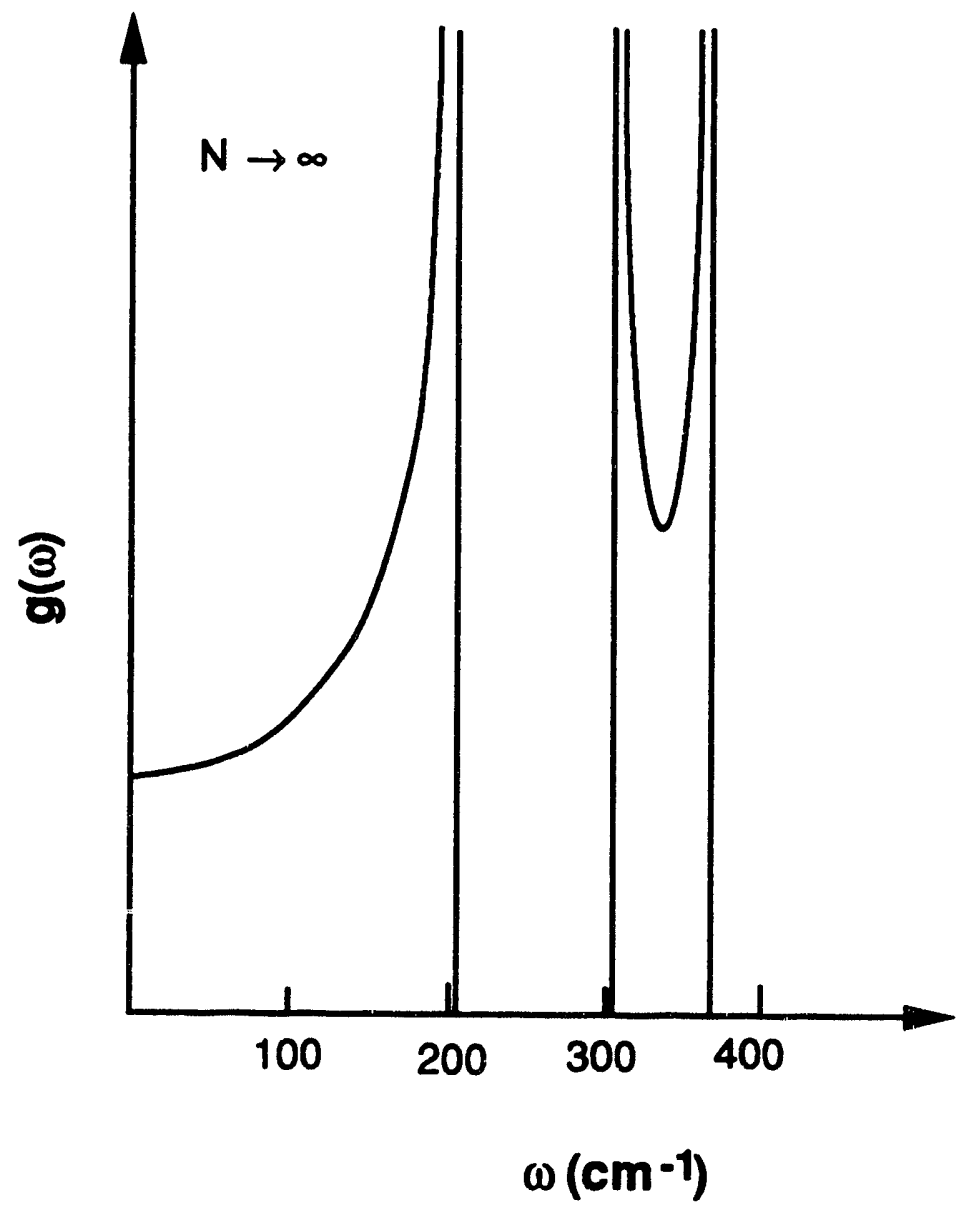

Fig 4.3. Density of states for diatomic chain shown in Fig.4.1 as number of atoms in chain approaches infinty. (Barker, 1975). 


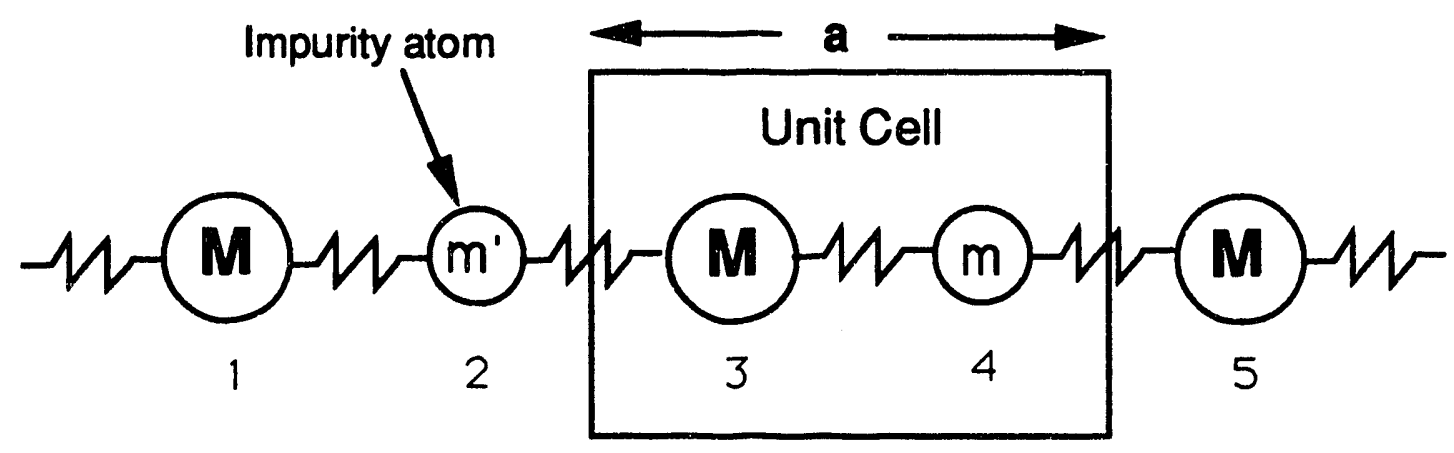

Fig. 4.4. Diatomic linear chain with nearest neighbor force constants and a light impurity $\left(m^{\prime}<m\right)$ replacing the lighter atom on the chain. (Barker, 1975). 


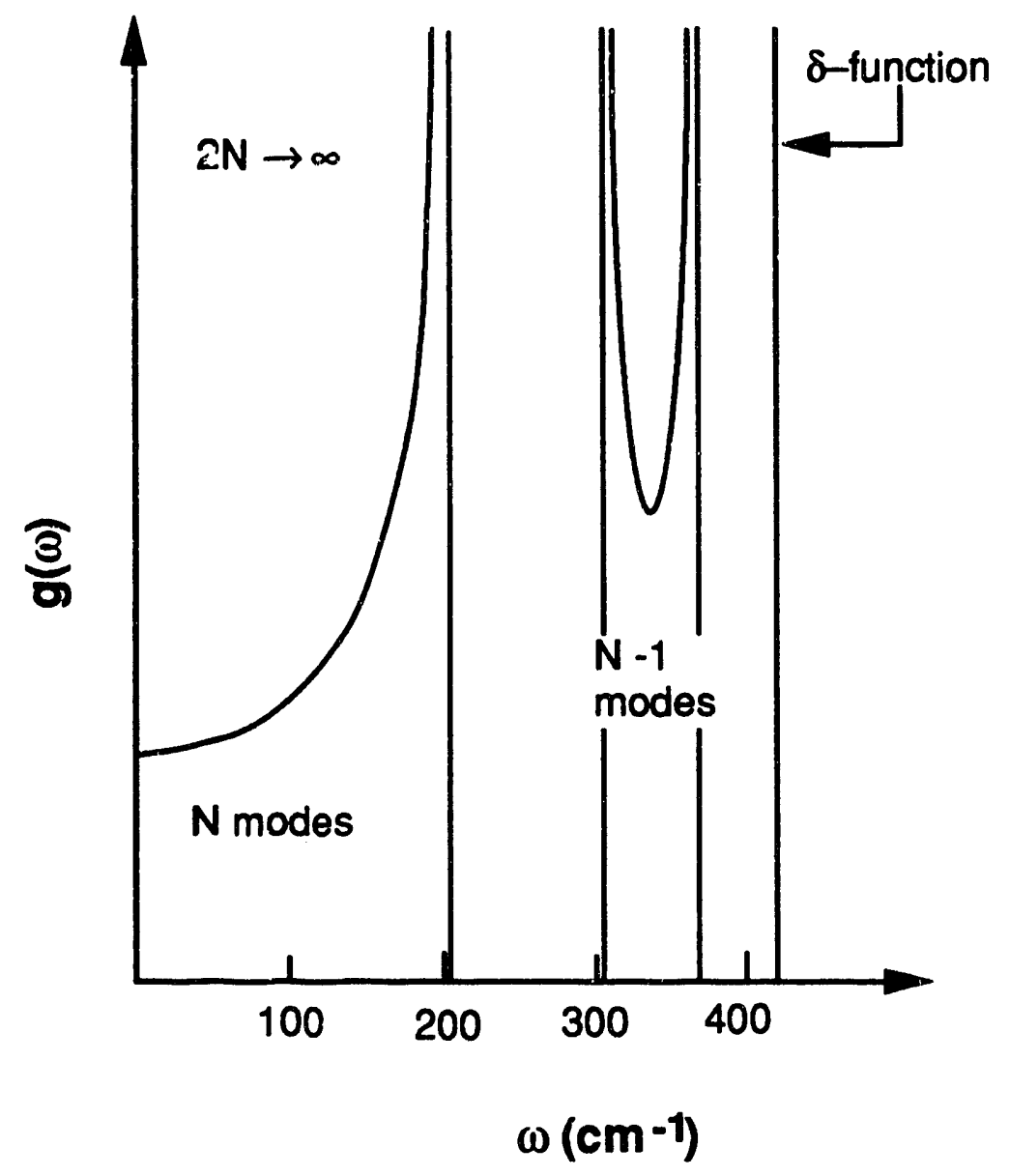

Fig 4.5. Density of states for diatomic chain of $2 \mathrm{~N}$ atoms $(\mathrm{N} \rightarrow \infty)$ with light impurity. $M=70 \mathrm{amu}, m=30 \mathrm{amu}$, and $\mathrm{m}^{\prime}=20 \mathrm{amu}$. (Barker, 1975). 


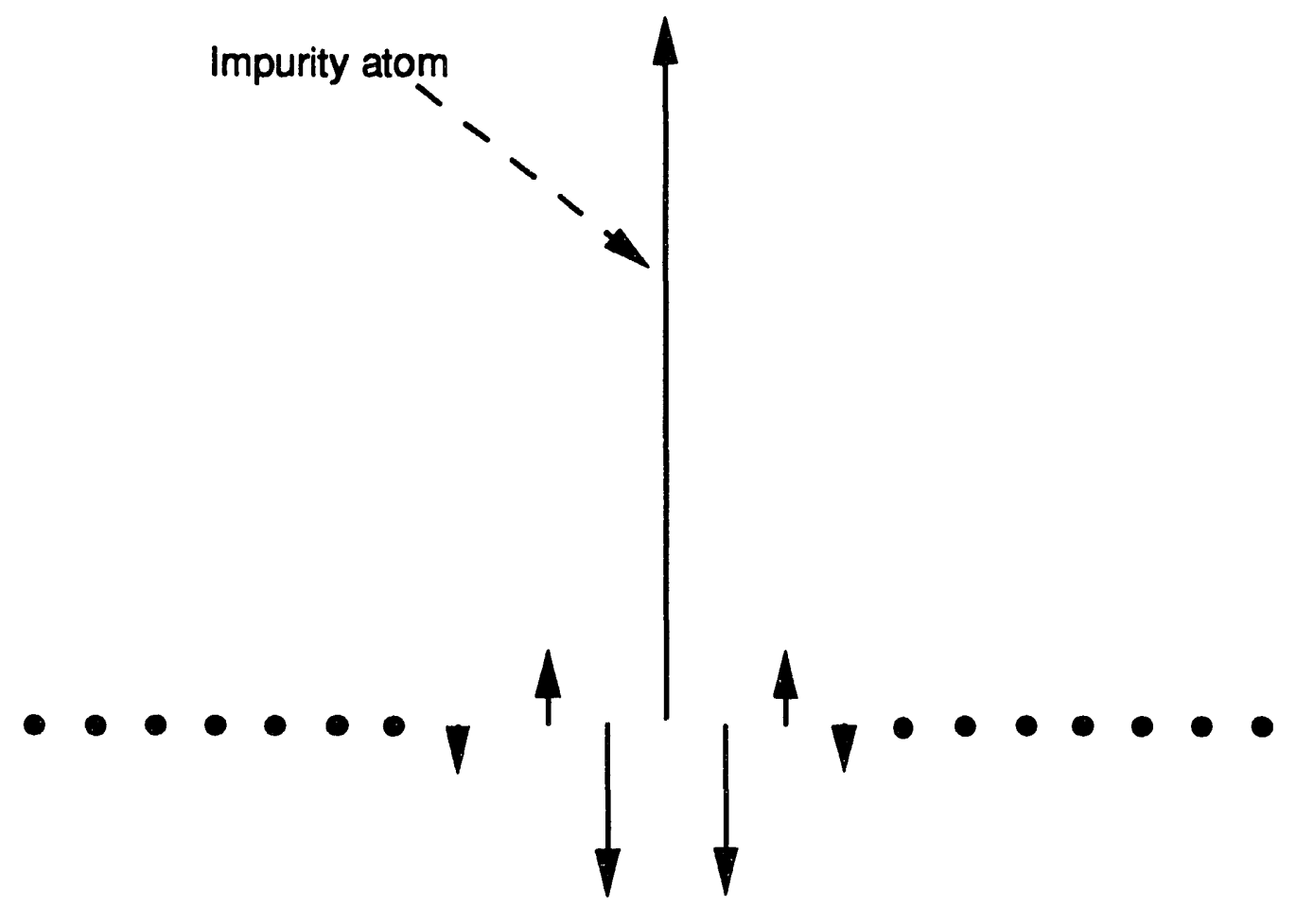

Fig. 4.6. Local vibrational mode of impurity on chain shown in Figure 4.4. Length of arrows represents displacement from equilibrium position. Atoms far from impurity don't move significantly. (Barker, 1975) 
$q$ is imaginary, indicating that the wave is damped for large deviations from the impurity site. It is also interesting to examine how the degree of localization depends on the impurity mass. As expected, a lighter impurity mass results in a greater degree of localization.

\subsubsection{Observation of Local Vibrational Modes}

Since the LVMs studied in this thesis were observed using Fourier transform spectroscopy this section will consist of a discussion of how light is absorbed by a local vibrational mode. We will focus on the vibrational mode of a Si impurity residing substitutionally on a Ga site in GaAs. Since GaAs crystallizes in a zincblende structure, the $\mathrm{Si}$ atom is bonded tetrahedrally to four nearest neighbor As atoms. In the spirit of the preceding section, we will model the $\mathrm{Si}$ atom as connected to the As atoms by a spring of force constant $\mathrm{k}$. We regard the positions of the all atoms other than the $\mathrm{Si}$ and the four nearest neighbor As atom to be fixed for the purposes of this discussion.

By solving the equations of motion, one can calculate the eigenfrequencies and eigenvectors for this system. Figure 4.7 shows two solutions to these equations, both of which represent LVMs of the (Si + As neighbors) system. The mode in $4.7 \mathrm{a}$ shows the $\mathrm{Si}$ atom moving against the As atoms while the mode in $4.7 \mathrm{~b}$, which is referred to as a breathing mode vibration, shows the $\mathrm{Si}$ atom to be stationary while the As atoms oscillate around it. These modes will clearly have different vibrational frequencies and so could be differentiated from each other experimentally. However, using Fourier transform spectroscopy, it is in fact only possible to observe the mode shown in

Fig.4.7a. The defect can only absorb light at its resonant vibrational frequency if the vibrational mode is infrared active. 


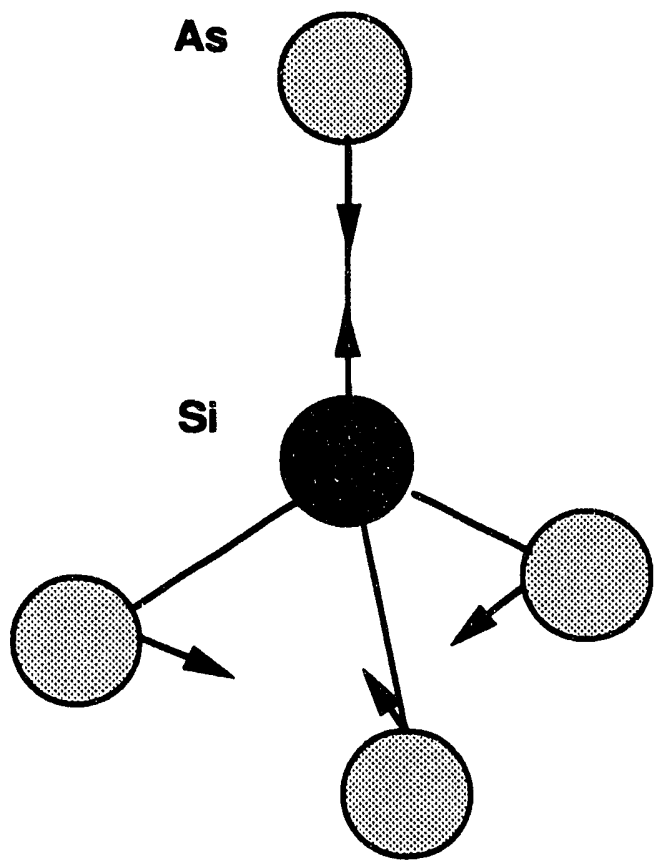

(a)

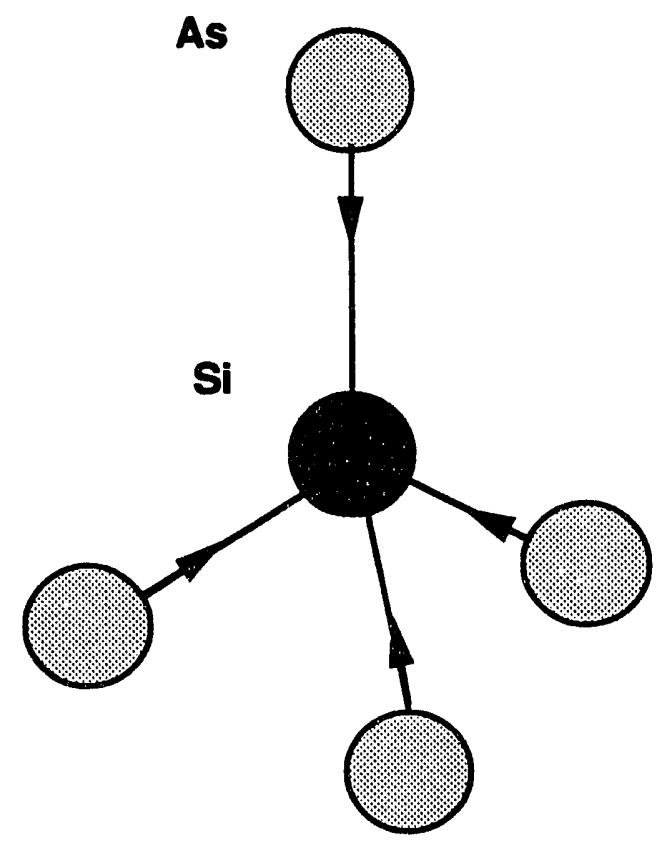

(b)

Fig. 4.7. Vibrational modes of Si-As system imbedded in GaAs lattice. Bonds to neighboring atoms are not shown and are assumed to be rigid. Only the mode shown in (a) is infrared active. 
The absorption intensity of an LVM is dependent on how well the energy of the incoming photon can be coupled to a vibration. This is in turn determined by the change in the dipole moment of the system in the presence of an oscillating electromagnetic field. For a simple system of a positively and negatively charged particle, the dipole moment is defined as the product of the magnitude of the charges and the spacing between them. For a more complicated system, one replaces the distance between the charges with the difference between the center-of-mass of the positive charges and that of the negative charges. The differing electronegativities of the $\mathrm{Si}$ and $\mathrm{As}$ atoms result in a transfer of charge between the atoms, leaving the As atoms with roughly three quarters of an electron more than the Si atom (Chadi, 1992a). The system has no net dipole though since the center-of-mass of the positive and negative charges are both located at the Si atom. Another way to look at this is to say that the four Si-As dipoles exactly cancel one another.

Absorption is due to a change in the dipole moment, however, and this can be induced by an incoming photon. Since the wavelength of infrared radiation is much larger than atomic spacings, the $\mathrm{Si}$ and $\mathrm{As}$ atoms all feel the same electric field. This means that the negatively charged As atoms will move in one direction while the positively charged $\mathrm{Si}$ atom moves in the opposite direction. This creates a separation between the center of mass of the positive and negative charges, changing the dipole moment from zero to a finite value. This type of motion is exactly the one pictured in Figure $4.7 \mathrm{a}$, and this mode is infrared active. We see that no change in the dipole moment occurs for the vibration pictured in Fig. 4.7b. The center of mass for both the positive and negative charges remains at the Si site throughout the vibration, implying that this vibration is infrared inactive. It turns out that this vibration can be observed 
using Raman spectroscopy, which is sensitive to the change in polarizability of a system in the presence of an electromagnetic field.

\subsection{Motivation for the Experiment}

Once Maude et al (1987) showed conclusively that the DX center was due to an isolated impurity, two basic questions remained about the microscopic structure of this defect: 1) What is the atomic arrangement of the donor impurity and its neighbors, and 2) What is the charge state of this defect. Progress on these questions has involved a fascinating interplay between theoretical and experimental progress. We first discuss the different theoretical models which have been proposed for this defect.

\subsubsection{Microscopic Models for the DX center}

\subsubsection{The Chadi and Chang Model}

Chadi and Chang (1988) proposed that DX centers form in AIGaAs and GaAs when a substitutional group IV donor breaks a bond with a nearest neighbor As atom and moves roughiy $1.2 \AA$ in the (111) direction, as shown in Figure 4.8. The figure also shows that, for a group VI donor impurity, it is the nearest neighbor $\mathrm{Ga}$ (or $\mathrm{Al}$ ) atom which is displaced. An interesting feature of this model is that these lattice relaxations are predicted to be unstable unless the defect is negatively charged. The reaction mechanism responsible for DX formation is proposed to be a two step process: 


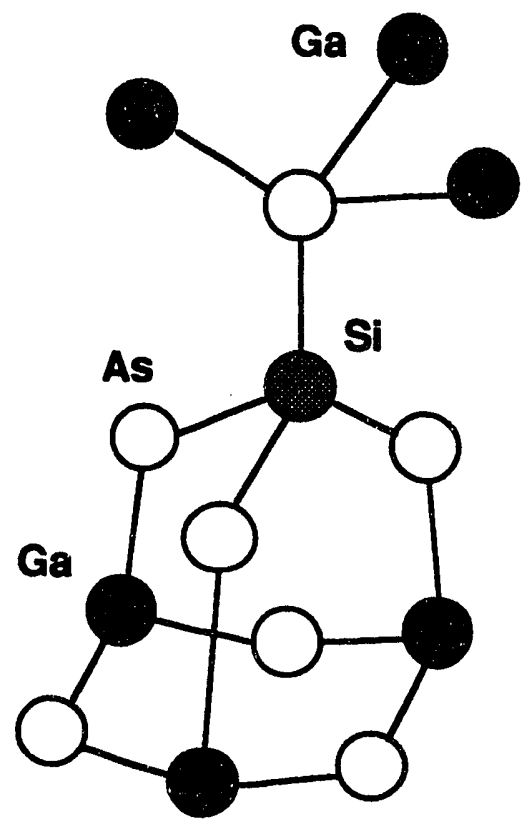

a) Shallow donor state

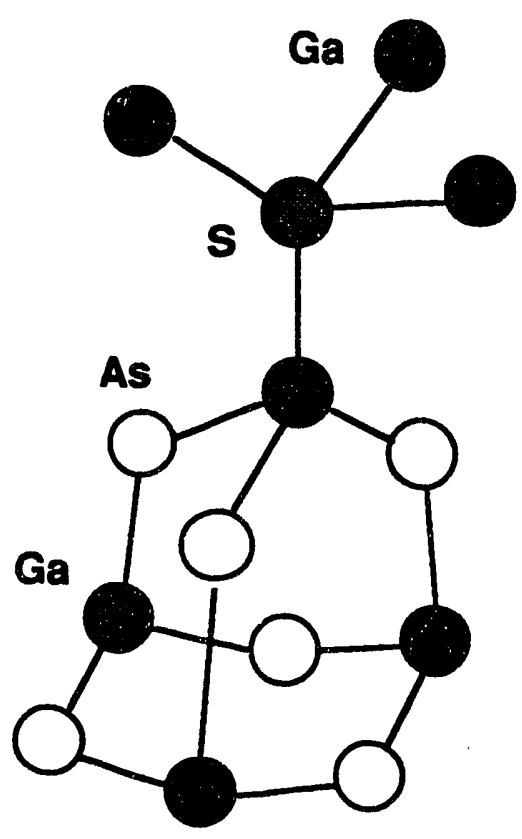

c) Shallow donor state

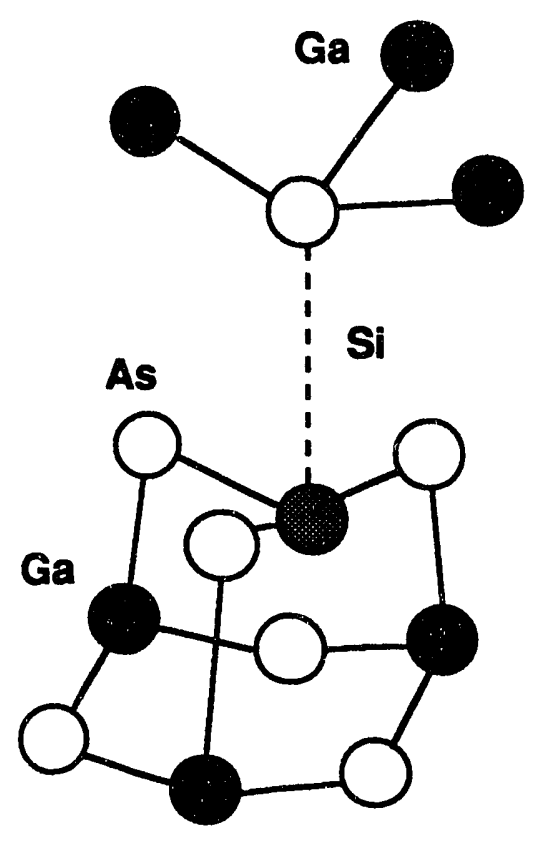

b) $\mathrm{DX}^{-}$state

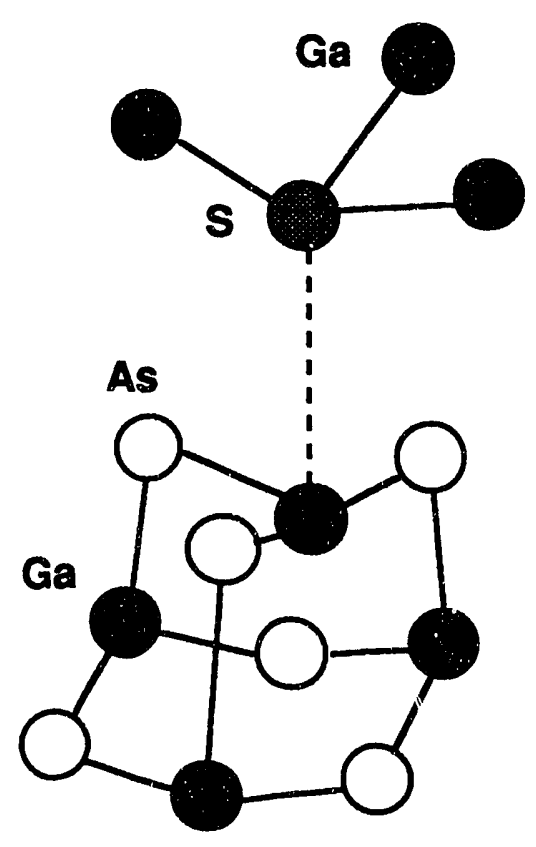

d) $\mathrm{DX}^{-}$state

Fig. 4.8. Chadi and Chang model for DX center formation. The figure shows that the atom which moves in the displacement depends on whether the impurity is a group IV $(a, b)$ or group VI $(c, d)$ atom. (Chadi and Chang, 1989) 


$$
\begin{aligned}
& d^{0} \rightarrow d^{+}+e^{-} \\
& d^{0}+e^{-} \rightarrow D X-
\end{aligned}
$$

The total reaction is

$$
2 d^{0} \rightarrow D X^{-}+d^{+}
$$

The first step is the ionization of a shallow donor, which requires roughly $6 \mathrm{meV}$, while the second step is exothermic. The lattice relaxation which results when the electron is captured by a neutral donor is calculated to decrease the energy of the defect more than it is increased by the Coulomb repulsion due to the presence of a second electron. This type of defect is referred to as a negative- $U$ defect, where $U$ is the Hubbard correlation energy (Anderson, 1975). It is defined as the difference between the Coulomb $e^{-}-e^{-}$repulsion and the stabilization energy due to the e--phonon interaction. A small number of such defects have been identified in semiconductors. The A center in Si (Watkins, 1961) and interstitial B in Si (Troxell, 1980) have been shown to be negative-U centers, and more recently it has been shown that the O-As vacancy complex in GaAs also exhibits this behavior (Alt, 1990; Skowronski; 1990).

Chadi and Chang also calculated the alloy and pressure dependence of the DX center binding energy. These predictions are based on their calculations which showed that the DX center is highly localized in real space, implying that it must be very spread out in momentum space. If this is the case, then the DX center wavefunction must be comprised of conduction band states from the entire Brillouin zone. This implies that the DX level should have an energy derivative with respect to alloying or pressure which is equal to that of the conduction band averaged over all k-space. A simple expression for the 
variation of the energy of the average conduction band, $\mathrm{E}_{\mathrm{CB}}$, is given by (Chadi, 1988)

$$
\Delta E_{C B}=\Delta[E(\Gamma)+3 E(X)+4 E(L)] / 8 .
$$

In addition to its simplicity, this expression is also extremely useful since the dependence of these minima on alloying and pressure are well known. Implicit in this expression is the fact that the impurity potential is not strong enough to require the consideration of higher lying conduction bands. We will return to this point in Chapter 5.

It was found experimentally that the binding energy of the DX level (Chand, 1985) roughly followed the $L$ conduction band minimum, and it was therefore postulated that the DX level was an L- derived level (Bourgoin, 1988). Using the above formalism it can be shown that this is not necessarily the case. Chand et al (1985) reported the alloy dependences of the conduction band minima in $A l_{x} G_{1-x} A s$ as

$$
\begin{aligned}
& \Delta E(\Gamma)=(1.42+1.25 \mathrm{x}) \mathrm{eV}, \\
& \Delta E(X)=(1.90+0.21 \mathrm{x}) \mathrm{eV}, \\
& \Delta E(L)=(1.71+0.64 \mathrm{x}) \mathrm{eV} .
\end{aligned}
$$

Inserting these values into Eq. 4.11 gives

$$
\Delta E(C B)=0.55 \times e V
$$

which is almost identical to the value of $0.54 x$ obtained by Chand et al. This result also clarifies the connection of this level to the $L$ band. The change in 
energy with alloying of the $\mathrm{DX}$ level $(0.55 \mathrm{x} \mathrm{eV})$ is close to that of the $L$ band $(0.64 \times \mathrm{eV})$, but it is now clear that this does not necessarily imply that the DX level is tied to the $L$ band.

The DX center will form when it is the most stable form of the donor, i.e., when its energy is lower than that of the shallow, substitutional donor. The eriergy of the shallow donor is tied to the $\Gamma$ conduction band minimum, so the DX level approaches the shallow donor level at the rate

$$
\Delta\left(E_{C B}-E_{\Gamma}\right)=(1.25-0.55) x=0.7 x \mathrm{eV} .
$$

Using the experimental fact that $D X$ levels form when $x \geq 0.22$, this implies that

$$
E(\Delta X)=\Delta E(\Gamma)+0.150 \times \mathrm{eV}
$$

meaning that in pure GaAs the DX level lies $150 \mathrm{meV}$ above the shallow donor level.

It is also possible to explain the observed maximum in the binding energy of the $D X$ centers observed in the allo; range $0.45 \leq x \leq 0.48$. From Eqs. 4.12, the $X$ band becomes the CBM when $X=0.46$. As the AlAs percentage is increased beyond this, the DX level is approaching the shallow donor level associated with the $X$ band at the rate

$$
\Delta\left(E_{C B}-E_{X}\right)=(0.55-0.21) \Delta x=0.34 \Delta x e V .
$$

The maximum in the DX binding energy should therefore occur at the $\Gamma-X$ crossover point, which is in fact what is observed. 
All of the behavior described in the preceding paragraphs can be summarized in Figure 4.9, which shows the variation of the various CBMs and the DX level as a function of alloying.

Analogous arguments can be made for the pressure derivative of the DX center energy. The pressure derivatives for the various conduction band minima are (Chang, 1984)

$$
\begin{aligned}
& \frac{d E(\Gamma)}{d p}=12 \mathrm{meV} / \mathrm{kbar}, \\
& \frac{d E(X)}{d p}=-1.6 \mathrm{meV} / \mathrm{kbar}, \\
& \frac{d E(L)}{d p}=5.5 \mathrm{meV} / \mathrm{kbar} .
\end{aligned}
$$

Using Eq.4.11 gives

$$
\frac{d E_{C B}}{d p}=3.6 \mathrm{meV} / \mathrm{kbar}
$$

implying that

$$
\frac{d\left(E(\Gamma)-E_{C B}\right)}{d p}=8.4 \mathrm{meV} / \mathrm{kbar}
$$

which is consistent with experimentally measured values. Since for $p=0, E_{D X}=$ $E_{\Gamma}+150 \mathrm{meV}$, this means that $\mathrm{DX}$ centers should form at pressures of approximately $19 \mathrm{kbar}$, which agrees reasonably well with the observed formation pressure in the range 20 - $30 \mathrm{kbar}$. The pressure dependences of the CBMs and DX level are shown in Figure 4. 10. 


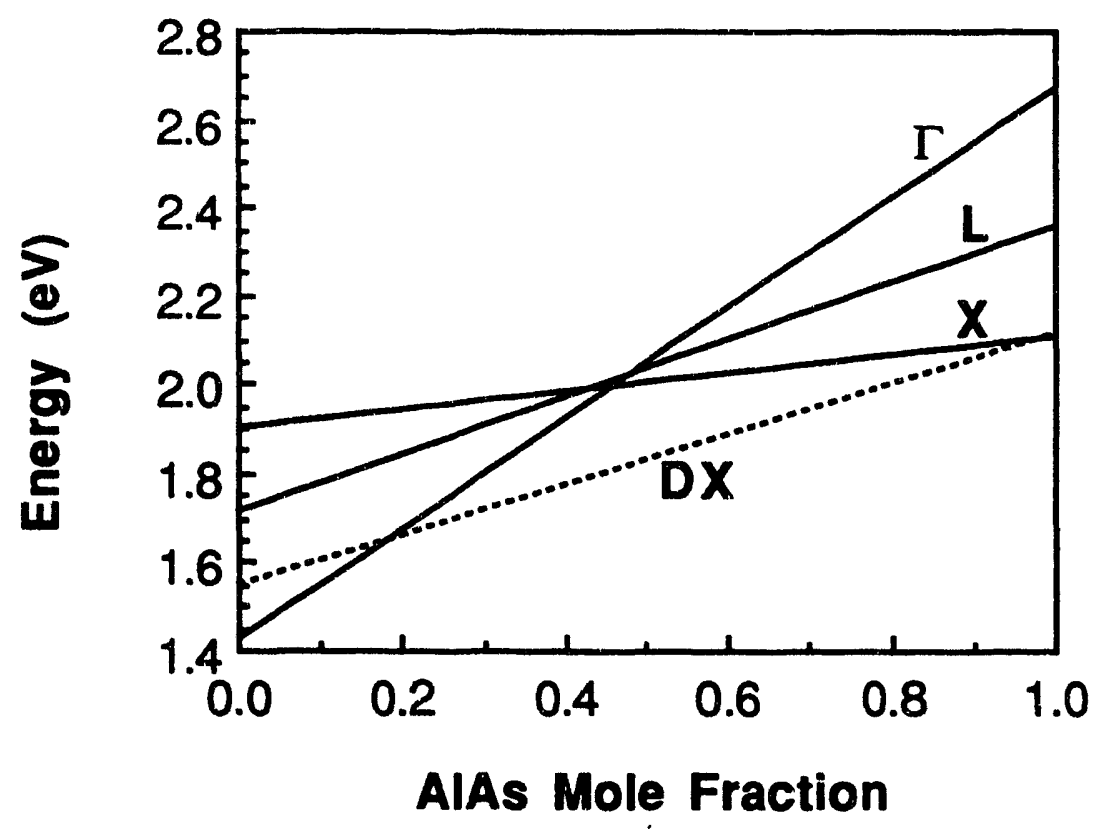

Fig. 4.9. Variation of the GaAs conduction band structure and the DX energy level as a function of AlAs alloying. 


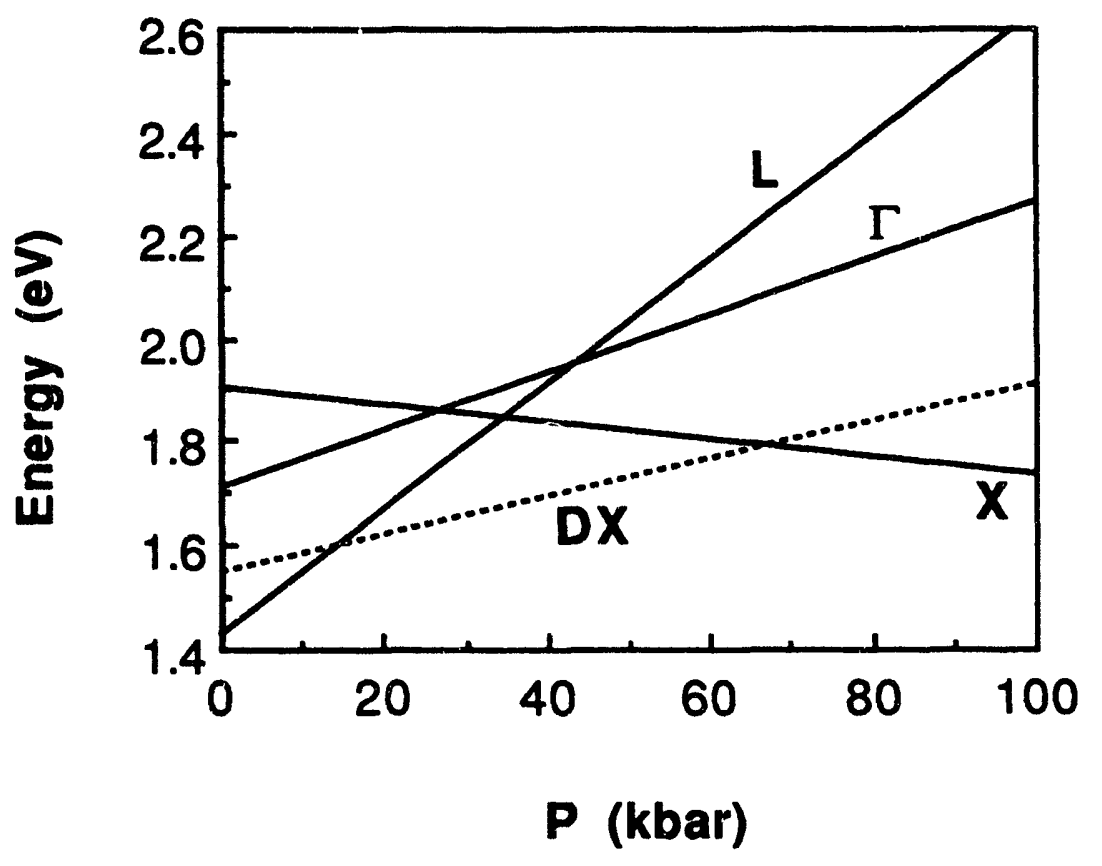

Fig. 4.10. Variation of the GaAs conduction band structure and the DX energy level with pressure. 
It must be stated at this point that although these arguments seem to describe the experimentally observed features of DX centers, they do not prove that the particular model proposed by Chadi and Chang is correct. These arguments for the pressure and alloy dependent behavior of the DX energy level work because the DX center is a localized state. Therefore, these arguments are valid for any model which predicts a localized nature for the DX center. The Chadi and Chang model does make two testable predictions, however. First, they claim that the charge state of the DX center is negative, and second, that the lattice relaxes into the broken bond geometry pictured in Figure 4.8. Attempts to test this model will be detailed later in this chapter.

A fascinating feature of this model is its prediction that the presence of a second electron actually creates the level at which it is subsequently trapped. This process is referred to as extrinsic self trapping, and has been described theoretically by Toyozowa (1978). We consider a conduction electron attracted to an impurity potential which is made up of short and long range forces. The following analysis will show that, depending on the relative strengths of the forces, there is a discontinuous decrease in the impurity radius down to the lattice constant $d$. The total energy of the bound electron can be written as

$$
E=B(d / a)^{2}-V(d / a)-U(d / a)^{3},
$$

where $V$ and $U$ represent the long (Coulomb) and short range potentials, respectively, and $a$ is the electron wavefunction radius. The first term represents the kinetic energy of the electron, where the coefficient $B$ is taken to be of the order of the width of the conduction band. The short range term is proportional to $(d / a)^{3}$ because only this fraction of the electronic charge feels the short range potential at the impurity site. 
We next consider electron-phonon interactions with long and short range forces. The long range force is taken to be the electrostatic interaction with the optical phonons in polar semiconductors. The effect of such a term on the electronic energy is given by $-E_{o p}(d / a)$ since the polarization energy due to the electronic charge present within the radius a is proportional to (1/a). The short range force is taken to be the deformation potential due to the acoustic phonons. The conduction electron is subject to the deformation potential $E_{d} \Delta(r)$, where $\Delta(r)$ is the local lattice dilation. The bound electron with radius a will distort the lattice within this region in order to minimize the sum of the deformation potential energy and the elastic energy, which is given by $a^{3} c \Delta^{2} / 2$, where $C$ is an elastic constant. Minimizing this sum with respect to a gives the stabilization energy to be $-E_{a c}(d / a)^{3}$.

The total energy of the defect-lattice system can then be written

$$
E=B(d / a)^{2}-\left(V+E_{o p}\right)(d / a) \cdot\left(U+E_{a c}\right)(d / a)^{3} .
$$

We now examine solutions to this equation as a function of the relative strengths of the short and long range forces. First we define short and long range force parameters $g_{s} \equiv\left(U+E_{a c}\right) / B$ and $g_{l} \equiv\left(V+E_{o p}\right) / B$. The next step is to minimize Eq. 4.21 with respect to (d/a), restricting solutions to the region $0<(d / a)<1$ since it is not expected that the impurity wavefunction radius should be less than a lattice constant. The possible solutions for different values of $g_{s}$ and $g_{1}$ are shown in Figure 4.11. This graph shows that there are four different regions of parameter space. In the region marked $L$, there is only one minimum and it occurs for $(d / a)=1$. In this region the defect is completely localized. In the region marked $D$, the minimum in energy occurs for $d / a=\left(1-\sqrt{1-3 g_{s} g_{1}} / 3 g_{s}\right)$, which in this range of parameter space means that $(d / a)<<1$, corresponding to a 


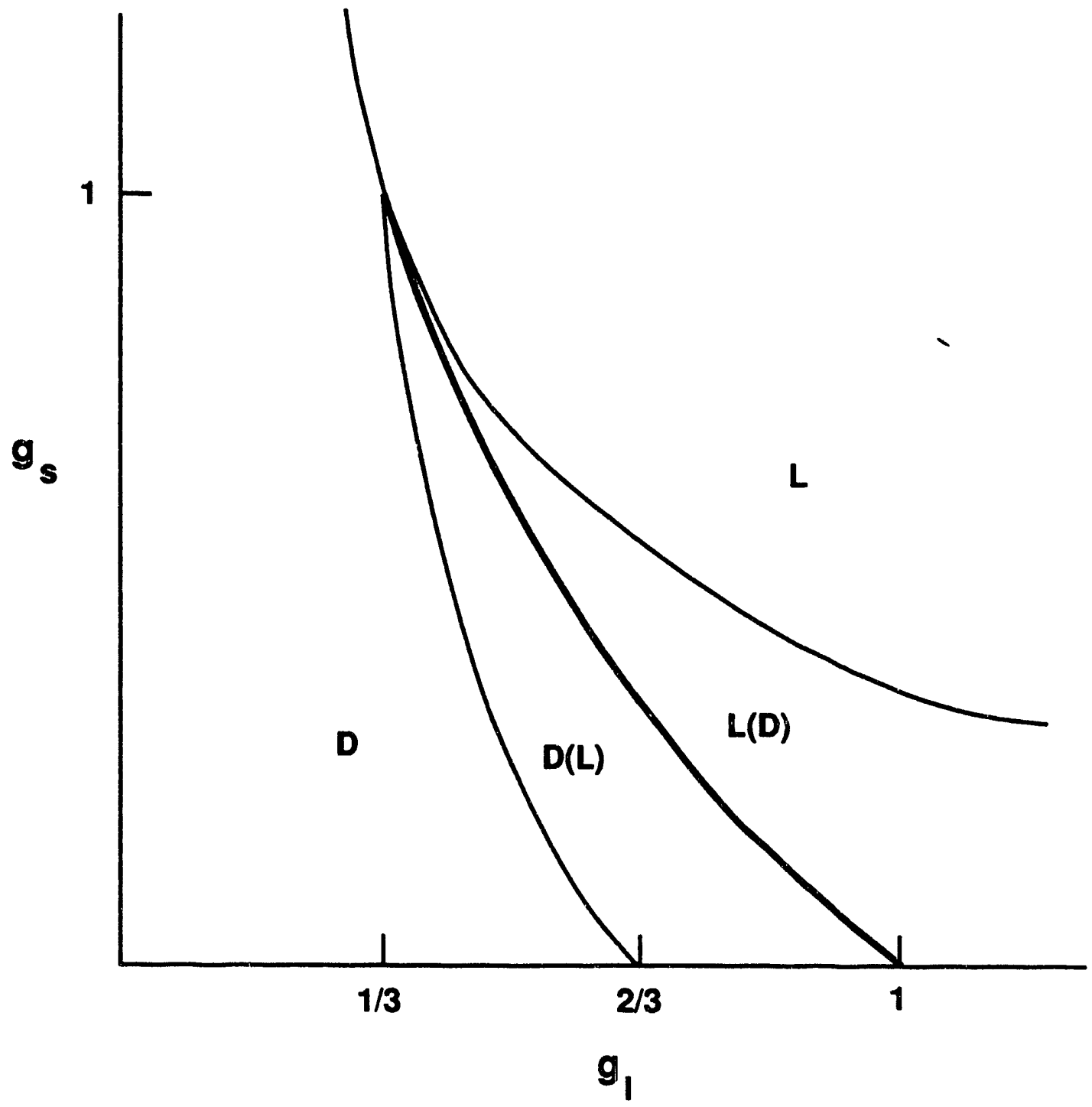

Fig. 4.11. Phase diagram for an electron in an impurity potential and interacting with phonons. The state in parenthesis is metastable. (Toyozowa, 1978). 
delocalized state. Crossing the thick solid line in the figure corresponds to the discontinuous collapse of the wavefunction from a delocalized to a highly localized state. In the region between the thin lines, there are two minima, one at $d / a=\left(1-\sqrt{1-3 g_{s} g_{1}} / 3 g_{s}\right)$ and one at $(d / a)=1$. In these regions, one of the solutions is stable while the other (labeled in parenthesis) is metastable. It is in this region that the DX level exists. Without the short range potential $U$ there would be no localized state. However, it is the combination of the long and short range forces which determines whether or not one obtains a localized or delocalized state, and whether or not the defect is metastable.

\subsubsection{The $A_{1}$ State}

It has also been proposed the $A_{1}$ state in GaAs can be identified with the DX center. This state can be most simply described in terms of tight-binding theory, and the starting place for this description is the four $\mathrm{sp}_{3}$ bonds formed by each atom with its nearest neighbors in the GaAs lattice. Fig. 4.12 shows that bonding and anti-bonding combinations of these orbitals can be formed, with the interaction of these levels broadening them into the valence and conduction bands, respectively. The figure shows that when a $\mathrm{Ga}$ atom is replaced with a $\mathrm{Si}$ atom, one creates bonding and anti-bonding combinations of the four Si-As bonds. Since $\mathrm{Si}$ is more electronegative than $\mathrm{Ga}$, the energy of these levels is lower than the corresponding levels in GaAs. Interaction between these levels splits them into a singlet $A_{1}$ state and a triply degenerate $T_{2}$ state, also as seen in Fig. 4.12. The $A_{1}$ state is resonant in the conduction band, and has been identified as a possible candidate for the DX center (Lanoo (1989); Yamiguchi, (1991)). 


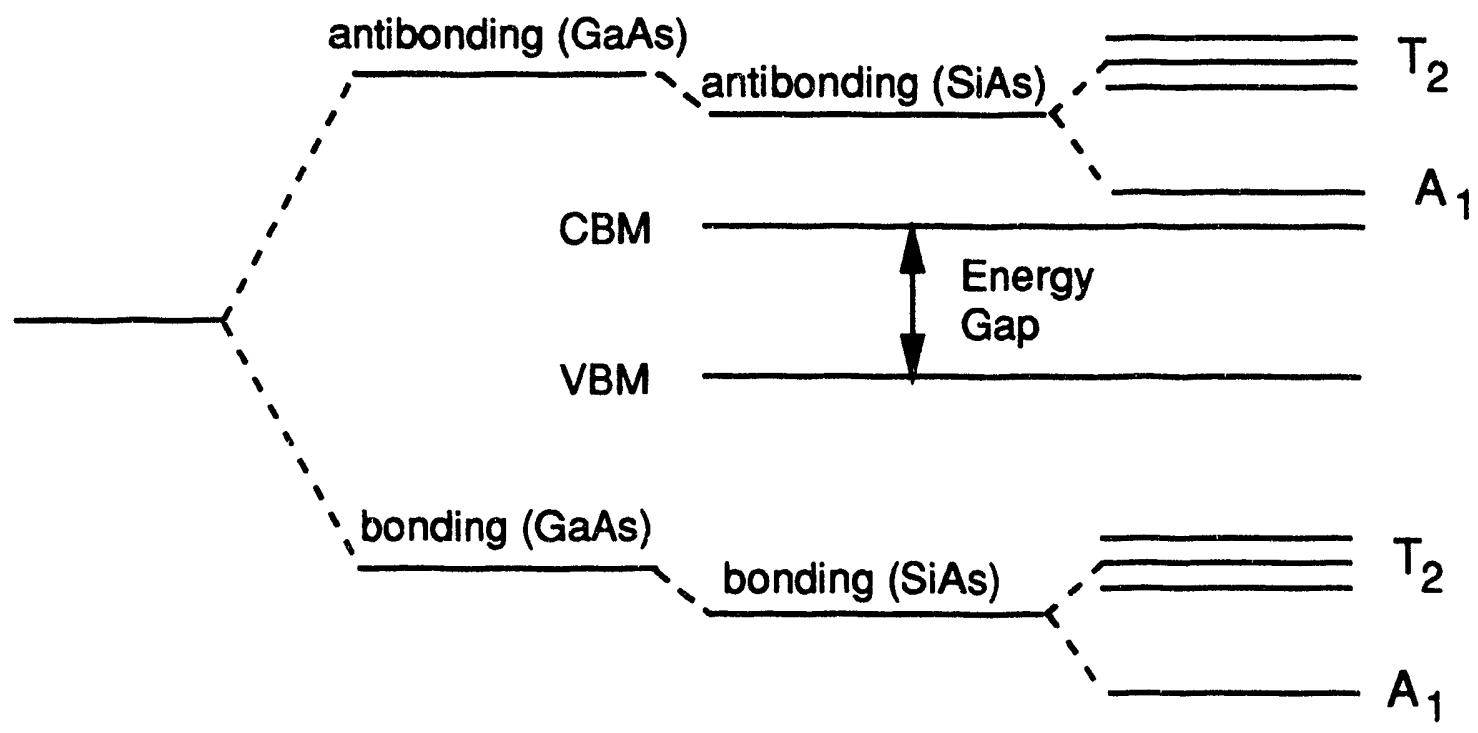

Fig. 4.12. Diagram showing the $A_{1}$ and $T_{2}$ bonding and anti-bonding donor states. CBM is the conduction band minimum and VBM is the valence band maximum. (Lannoo, 1990). 
In order to test this idea, a self-consistent pseudopotential calculation was performed by Yamiguchi et al (1991). In this calculation, a large number of possible configurations were tested for their relative stability. Their conclusion was that the most stable form of the donor was a deep $\mathrm{A}_{1}$ state in which the $\mathrm{Si}$ sits substitutionally while the nearest neighbors relax. The relaxation of the first and second nearest neighbors is shown in Table 4.1 for GaAs:Si at various pressures. $Q_{1}$ represents the change in the Si-As nearest neighbor bond length, while $Q_{2}$ and $Q_{2}$ represent the change in the second nearest neighbor bond length and bond angle, respectively. Yamiguchi et al also examined the energetics of the relaxation proposed by Chadi and Chang, and found that this was a metastable state with an energy roughly $600 \mathrm{meV}$ larger than that of the $A_{1}$ state. This is obviously in direct contradiction to the Chadi and Chang calculation. Yamiguchi et al also showed how the $A_{1}$ state level starts off resonant in the conduction band but comes into the gap with pressure or alloying.

Since this theory implies that a large lattice relaxation is not responsible for DX center formation, it must provide explanations for 1) persistent photoconductivity and 2) the large optical ionization energies which are observed. To explain persistent photoconductivity, Yamiguchi et al refer to the results of a calculation by Hjalmarson (1984) which shows that the radiative recombination efficiency of electrons falling from the $\Gamma$ band to the $A_{1}$ state is 10-22 times less than recombination from $X$ or $L$ band donors. The barrier found for destruction of persistent photoconductivity is therefore the barrier between the $\Gamma$ band and these other extrema. Yamiguchi et al also claim that the optical ionization measured for $D X$ centers is the transition from the $A_{1}$ state to the $T_{2}$ state, the energy of which they calculate to be $0.95 \mathrm{eV}$, in rough agreement with experimental values. However, there are serious objections to this identification. 
Table 4.1. - Values of $Q_{1}, Q_{2}$, and $Q_{2}$ for the minimum defect energy in GaAs:Si according to the calculation of Yamiguchi (1991).

$\begin{array}{llll}\text { P(kbar) } & Q_{1}(\%) & Q_{2}(\%) & Q_{2}^{\prime}(\%) \\ 0 & +0.91 & +0.83 & +0.26 \\ 30 & +0.70 & +0.80 & +0.25 \\ 60 & +0.50 & +0.77 & +0.24\end{array}$


They made arguments as to why the transition probability to conduction band minima, which would start at much lower energies, are much less probable than the $A_{1}-T_{2}$ transition. However, Baraff (1991) showed that these arguments were incorrect. It must be stated therefore that the $A_{1}$ state model does not effectively explain the large photoionization threshold energy.

A third calculation was carried out by Dabrowski et al (1990). They found both the $A_{1}$ state and the broken bond state, but were unable to calculate which was lower in energy. It is clear that experiments are needed to decide which theoretical model is closest to the truth. It should also be mentioned that a more recent calculation by Chadi (1992b) does predict the existence of the $A_{1}$ state, kut claims that it is higher in energy than the broken bond state and is in fact also negatively charged. He also claims that the lattice relaxation is larger than that calculated by Yamiguchi and that it would in fact exhibit persistent photoconductivity.

\subsubsection{Summary of Previous Experimental Work on DX Centers}

There have been a large number of experiments performed on the DX center in the past decade, and this section will focus on experiments which attempted to delineate which of the models described in the previous section is correct. These can be split into two categories 1) those which attempt to determine the lattice relaxation of the DX center, and 2) those which attempt to determine the charge state of the DX center.

Many experimenters have tried to find evidence of a lattice relaxation in the neighborhood of the DX centers. In one of the most convincing experiments, deep level transient spectroscopy (DLTS) was used to observe $A l_{x} \mathrm{Ga}_{1-x} \mathrm{As}: \mathrm{Si}$ samples which had different alloy percentages (Mooney,1988). In pure GaAs 
only one peak was observed. However, as the AlAs percentage was increased, several closely spaced peaks were observed whose energies did not depend on $x$ but whose relative intensities did. This result was interpreted as demonstrating that the $\mathrm{Si}$ atoms had moved to interstitial positions where the average number of $\mathbf{A l}$ neighbors they had was a function of alloy content. As many as four peaks have been observed, which would be consistent with zero, one, two, or three nearest neighbor Al atoms. In a clever variation of this experiment, $A l_{x} \mathrm{Ga}_{1-x} \mathrm{As}$ :Te were studied in a similar fashion (Dobaczewski, 1992). If the above interpretation is correct, then one would expect up to eight peaks in highly alloyed samples, since in this case either a Ga or Al atom would move, resulting in two series of four peaks. This is precisely what is observed.

Many other experiments suggest the presence of a large lattice relaxation in the formation of DX centers. A study by Northrop et al (1991) showed that there was no evidence of any DX center photoionization below 0.8 $\mathrm{eV}$, and this cannot be explained by a small lattice relaxation model, as was argued in the discussion of the $A_{1}$ state (Section 4.2.2b). It has also been demonstrated that the pressure coefficients of the ionization energy and capture barrier height of the DX center in GaAs:Si are only consistent with a large lattice relaxation ( $L i, 1987)$. Recent $x$-ray diffraction experiments also show that $D X$ formation is (Cargill,1992; Leczinski,1991) accompanied by an increase in the lattice constant. EXAFS measurements found no evidence for a change in the nearest neighbor distance for $\mathrm{Sn}$ and $\mathrm{Se}$ substitutional donor atoms (Hayes, i989; Kitano, 1987), but the interpretation of these results is not straightforward. In the Chadi and Chang model, the Sa atom would not move and the Sn atom would move to a position such that its bond lengths are very similar to those in the undistorted substitutional position. 
Other experimenters have attempted to determine the charge state of the DX center. An ingenious set-up was used by Fujisawa (1989) to show that the DX center is negatively charged. In this experiment, deep level transient spectroscopy was performed on samples co-doped with germanium and silicon under hydrostatic pressure. Ge transforms into DX centers at a lower pressure than $\mathrm{Si}$, so below the $\mathrm{Si}$ transformation pressure the $\mathrm{Si}$ atoms act as a source of free electrons for Ge DX center formation. The experiment showed that the number of electrons trapped at the $\mathrm{Ge} D X$ centers scaled with the Si concentration aris aturated at roughly twice the concentration of uncompensated $\mathrm{Ge}$ atoms, demonstrating that two electrons were trapped at each Ge DX center.

Mössbauer experiments on GaAs:Sn have also been interpreted as being consistent with the localization of two or three electrons at each DX center (Gibart,1990). Photoionization experiments by Dobaczewski et al (1989) have also been shown to be consistent only with a negatively charged DX center. Recent studies of the capture and emission kinetics of DX centers (Theis, 1991; Mosser,1990) also show that observed equilibrium occupations of DX centers cannot be understood if only one electron is captured or emitted.

A seemingly straightforward technique for determining the charge state of the DX center is to look at changes in mobility after photoionization. Roughly speaking, if the DX center is negatively charged, then there is no net change in the ionized donor concentration upon DX center formation and there should be no change in mobility. If it is neutral, then there are more ionized centers and there should be a decrease in mobility due to additional ionized impurity scattering. This analysis turns out to be far too simplistic, however. It has been hypothesized (O' Reily, 1989; Williamowski, 1991) that there is a correlation between the location of the ionized shallow donors and DX centers which leads 
to relatively weak dipole scattering of electrons. Changes in the Fermi level must also be taken into account (Chadi, 1989a). Because of these and other complexities the analysis of mobility data does not provide any definitive conclusions.

An experiment whose results are in conflict with the negative-U model used magnetic susceptibility measurements to show that DX centers in several approximately $10 \mu \mathrm{m}$ thick epilayers of $A l_{x} \mathrm{Ga}_{1-x} \mathrm{As}(x>0.23)$ doped with $\mathrm{Si}$ or $\mathrm{Te}$ were paramagnetic, implying that only one electron was bound (Khachaturyan,1989a). However, a similar experiment performed on a $200 \mu \mathrm{m}$ thick epilayer of $\mathrm{Al}_{0.3} \mathrm{Ga}_{0.7} \mathrm{As}: \mathrm{Te}$ which had its substrate removed found that the concentration of paramagnetic centers was an order of magnitude less than the concentration of DX centers (Katsumato, 1990). There has yet to be a resolution of these conflicting results.

\subsection{Local Vibratianal Mode Spectroscopy of DX Centers in GaAs under} Hydrostatic Pressure

Although it is obvious from the preceding discussion that a great many techniques have been used to try to determine the structure of the DX center, one of the most obvious experiments had yet to be attempted. If some form of lattice relaxation takes place when a substitutional donor transforms into a DX center, then the bonding of the donor to its nearest neighbors must change and the frequency of the donor LVM should also change.

The most straightforward way of performing this experiment would be to try to observe the DX LVM in epilayers of AlGaAs. However, the vibrational spectra of alloys are extremely difficult to interpret because variations in the local environment of the defect lead to substantial broadening of LVM peaks. An 
even larger problem exists in attempting to study DX centers in this manner. $\mathrm{Si}$ is the only donor which may be used for these studies, since it is the only one which has an observable LVM (One would suspect that $S$ could also be used since it is lighter than both $\mathrm{Ga}$ and $\mathrm{As}$, but there has beer, no LVM observed for this impurity (Beall, 1985)). Unfortunately, Si has a mass very close to that of Al, which implies that its vibrational frequency is not very different. So, even in AlGaAs with very small AlAs fraction, the amou. $\mathrm{Al}$ is two orders of magnitude larger than the Si concentration in even a highly doped $\left(10^{18} \mathrm{~cm}^{-3}\right)$ sample. It is thus seen experimentaliy that the Al LVM peak completely overwhelms that due to the Si (Mooney,1991).

If pressure were to be employed to avoid the use of alloyed samples, then the sample would have to be placed in a diamond anvil cell (DAC). No one had previously attempted to measure the DX LVM frequency by this method because of the difficulties described in Section 3.1.4. These difficulties were overcome using the set-up described in that chapter, and the present chapter describes the first observation of an LVM of the Si substitutional donor and DX center in GaAs under hydrostatic pressure up to 40 kbar (Wolk, 1991b; Haller, 1991).

The experiment can be divided into two main parts. First, a new local vibrational mode observed in hydrostatically stressed GaAs:Si is identified as an LVM of the Si DX center. The spectroscopic data is then combined with Hall effect and resistivity analysis to determine the charge state of the DX center.

Before continuing with the description of the experiment it is necessary to make a brief digression to discuss free carrier absorption in semiconductors. Because the Bohr orbit of donor impurities in GaAs is $\approx 100 \AA$, the impurity wavefunctions overlap at concentrations above a few times $10^{16} \mathrm{~cm}^{-3}$. This results in the formation of an impurity level band, which is so broad that it 
merges with the conduction band. Under these conditions, all impurity donors contribute carriers to the conduction band at all temperatures. This results in free carrier absorption which makes the samples opaque to far infrared radiation. Free carrier absorption results occurs when a carrier absorbs a photon and is taken into a higher energy state in the same band. Additional momentum is required since only states away from $k=0$ are available, and it is provides by a scattering process with either acoustic phonons, optic phonons, or ionized impurity scattering. All the samples studied in this work were doped heavily enough that the ionized impurity absorption scattering process was dominant. The high concentration of free carriers made the sample completely opaque in the far infrared (The absorption cross section for ionized impurity scattering free carrier absorption is proportional to $\lambda^{3.5}$ ), which makes absorption spectroscopy impossible.

In order to overcome this difficulty, various techniques have been devised. One method is to use defect passivation. In this technique, a fast diffusing species, $\mathrm{Li}$, for example, is introduced into the sample. It forms complexes with the donors which eliminate their electrical activity (Barker, 1975). This technique has the disadvantage that the passivating species often forms complexes which themselves have LVMs, and this complicates the interpretation of the absorption spectra. It is also true that in some materials it is difficult to find an element which both diffuses quickly and passivates.

Another technique used to make heavily doped samples transparent is bombardment by electrons of roughly $1 \mathrm{MeV}$ in energy. The irradiation creates electronic levels near the middle of the band gap which compensate all free carriers (Theis, 1984). A quick calculation shows that the damage introduced does not affect the LVMs of the donor atoms. The dose used in the irradiation of GaAs is enough to produce the same concentration of compensating defects as 
donor impurities. At a concentration of $1018 \mathrm{~cm}^{-3}$ the average volume containing a single impurity is $10^{6} \AA^{3}$. The damage introduced by the electrons is extremely localized. Using very approximate numbers, let us say that volume associated with an irradiation defect is roughly the same as that of an LVM, which is approximately $(4 \pi / 3)(10 \AA)^{3} \approx 4000 \AA^{3}$. This implies that only one out of every $\left(10^{6} / 4 \times 10^{3}\right)=250 \mathrm{LVMs}$ will be influenced by the point damage due to the irradiation, and this lack of any observable broadening of LVM peaks has been borne out experimentally (Theis, 1984).

We now discuss the observation of the Si LVMs in GaAs under pressure. In order to determine if some change in the frequency of the impurity LVM occurs upon transforming into a DX center, it is first necessary to determine where the mode of the untransformed substitutional donor lies under pressure. This was done using a piece of GaAs:Si with $\mathrm{nSi}_{\mathrm{Si}}=6.3 \times 10^{17} \mathrm{~cm}^{-3}$, subsequently referred to as Sample 11. This sample had been irradiated with $1 \mathrm{MeV}$ electrons, making it transparent to infrared radiation at all pressures. Whether the DX center is neutral or negative, its formation is suppressed in an electron irradiated sample since there are no free electrons available. A spectrum of Sample 11 in the DAC taken at $P=35 \pm 2$ kbar is shown in Figure 4.13. One peak is seen whose frequency can be observed as a function of pressure. Spectra at different pressures are shown in Figure 4.14 and the data for all samples we observed are summarized in Figure 4.15. This peak may be unambiguously identified as the Siga LVM since its frequency at zero pressure matches what has been previously observed for the substitutional donor (Woodhead, 1985). The dependence of the LVM frequency on pressure is linear over the range of this study and is given by $d v_{S i G a} / d p=0.66 \pm 0.03 \mathrm{~cm}^{-1} / \mathrm{kbar}$. 


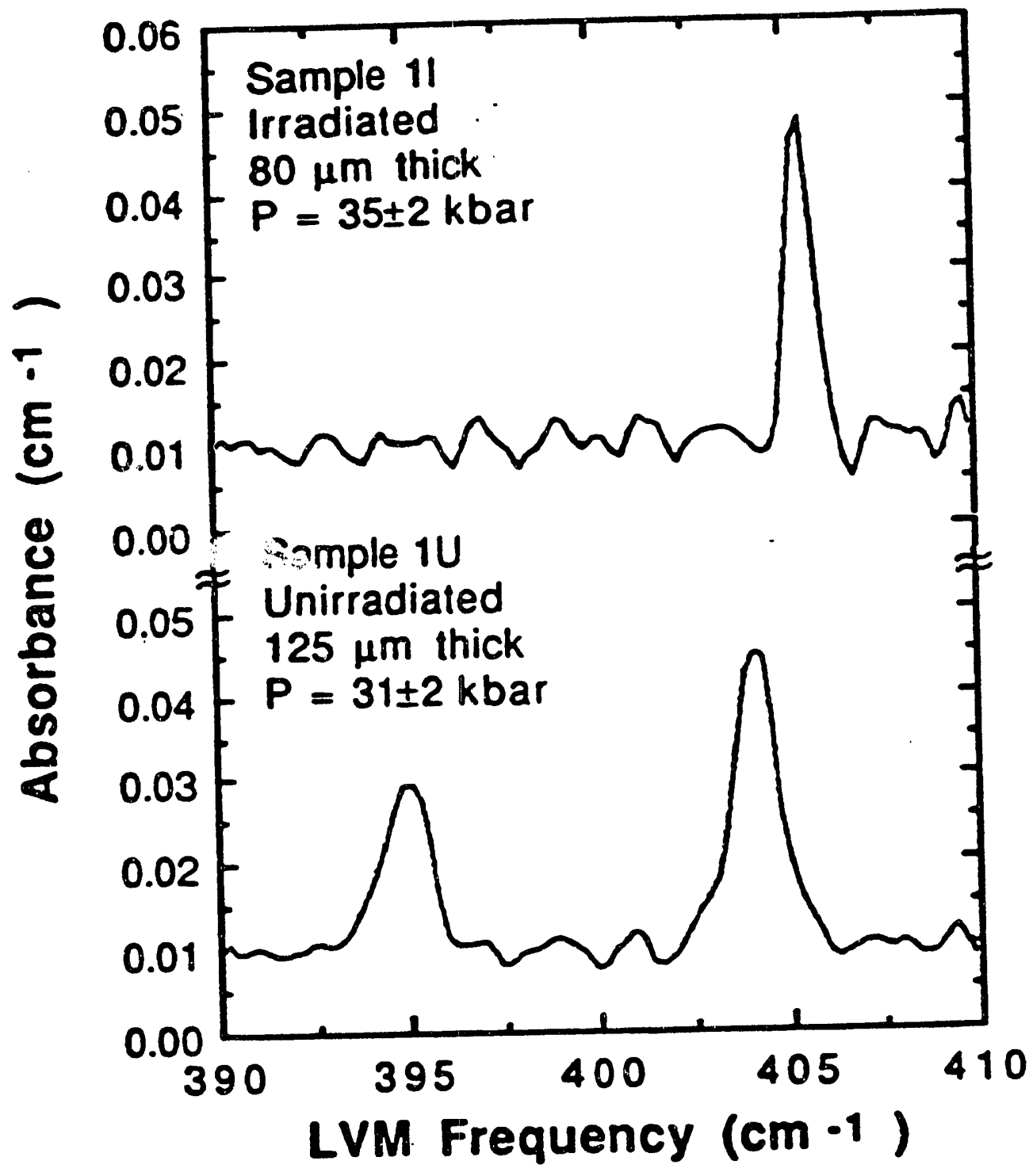

XBL 9011-3565

Fig. 4.13. Absorption spectra of samples 11 and $1 U$ at $T=5 \mathrm{~K}$. 


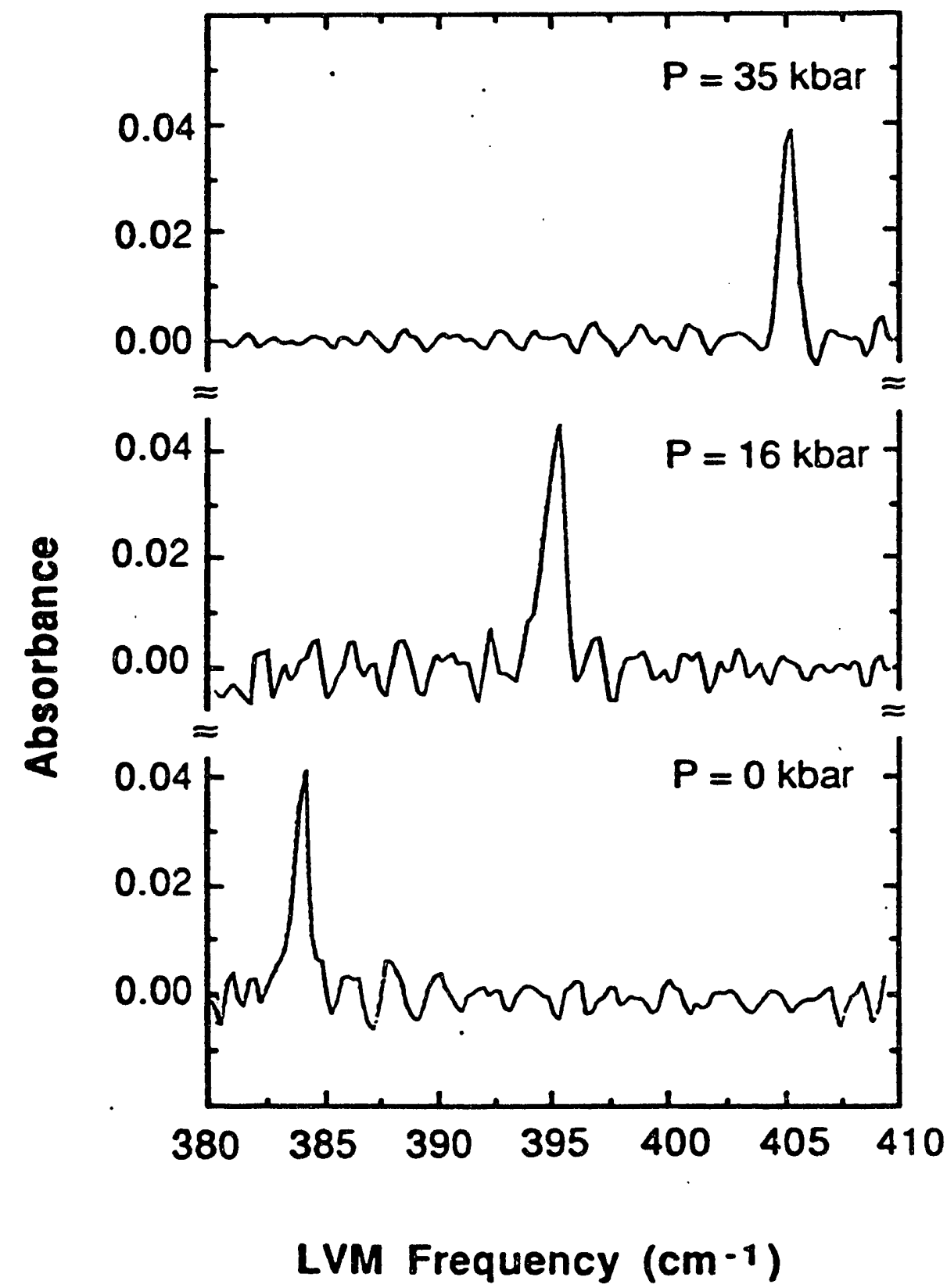

XBL 912-189

Fig. 4.14. Absorption spectra of sample $1 /$ at three different pressures. $T=$ $5 \mathrm{~K}$. 


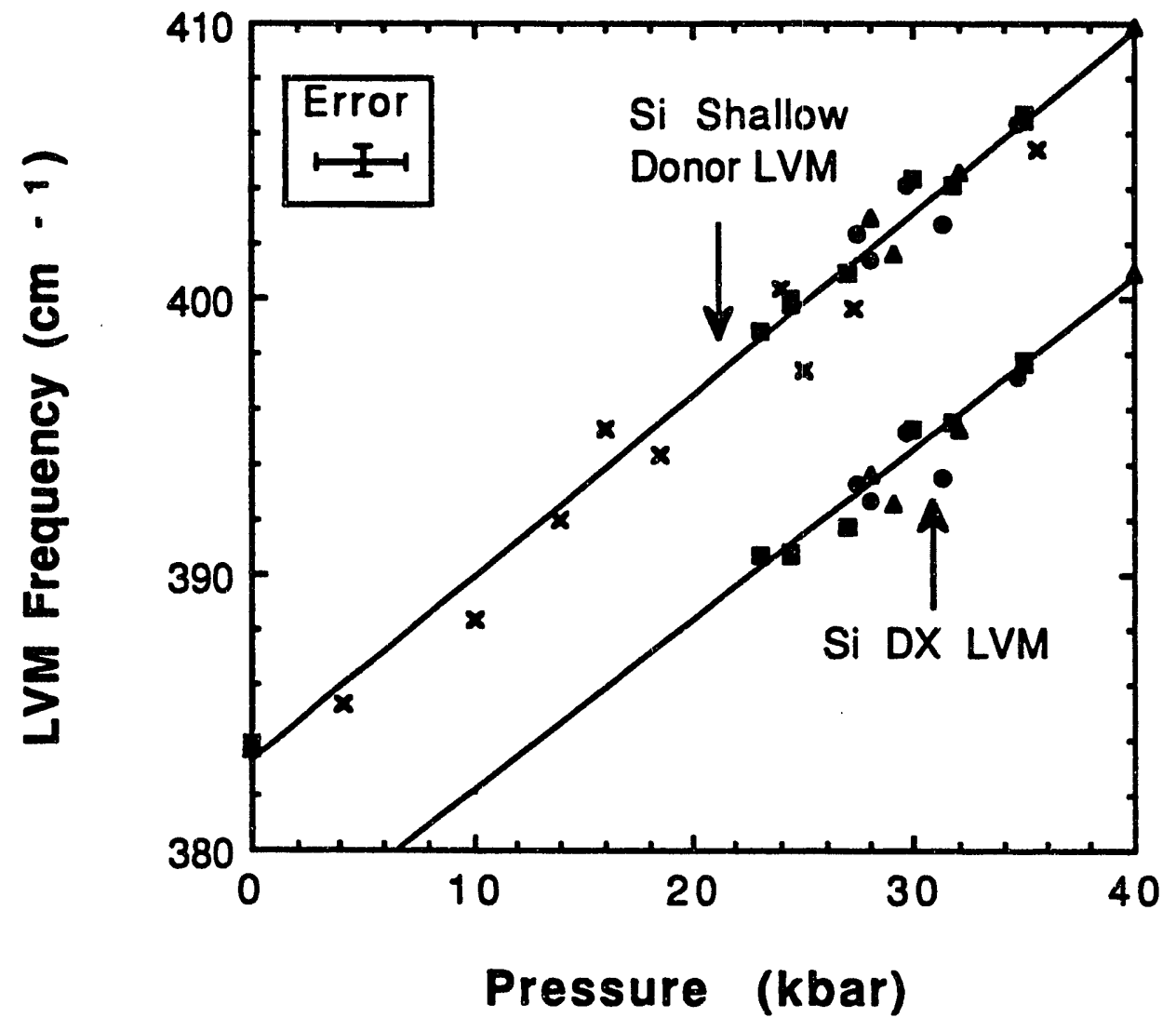

Fig. 4.15. Pressure dependence of the SiGa and Sidx LVM frequencies. $x$, - . and $\Delta$ refer to samples $11,1 U, 2$, and 3, respectively. 
Figure 4.13 also shows the spectrum of Sample $1 \mathrm{U}$ at $30 \pm 2 \mathrm{kbar}$, which is well above the pressure necessary to cause the DX transformation. This sample is identical to Sample 11, having been cut from the same part of the same wafer, except that it had not been irradiated. There are two peaks clearly observed in the spectrum, one at $404 \mathrm{~cm}^{-1}$ and the other at $395 \mathrm{~cm}^{-1}$. The peak at $404 \mathrm{~cm}^{-1}$ is precisely where the SiGa LVM is expected at $31.5 \mathrm{kbar}$, but the peak at $395 \mathrm{~cm}^{-1}$ is a new feature. The pressure dependence of this new feature is shown in Figure 4.14 and is $d v / d p=0.61 \pm 0.04 \mathrm{~cm}^{-1} / \mathrm{kbar}$, roughly the same as that of the Siga peak. There is no data below 23 kbar because the sample is opaque below this pressure due to free carrier absorption. This new feature will be identified as the LVM of tho Si DX center.

As a first step in making this identification, we confirmed that DX centers were present in the sample by performing the following series of experiments, which are outlined in Figure 4.16. First, the sample was brought to a pressure high enough to form DX centers. The sample was then brought to $4 \mathrm{~K}$ and illuminated with white light. The amount of the infrared radiation reaching the detector was drastically reduced because of free carrier absorption resulting from the photoionization of the DX centers, and we were unable to obtain an absorption spectrum of the sample. The carriers remain in the conduction band as long as the sample is kept at low temperature due to the capture barrier against return to the DX level. The sample was then brought to room temperature and recooled at a pressure of only $21 \mathrm{kbar}$, too low to cause the DX transformation. Once again, no spectrum of the sample could be taken. Bringing the sample back to room temperature and increasing the pressure to $24 \mathrm{kbar}$ resulted in the sample once again being transparent.

In heavily doped GaAs:Si samples, many LVM peaks in addition to the Siga peak have been observed with FIRFTS (Woodhead, 1985). They have 


\section{Action \\ Result \\ Explanation}

Apply pressure to sample greater than 23 kbar. Cool to $5 \mathrm{~K}$.

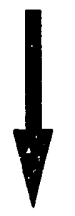

Shine light on sample and keep at low temperature.

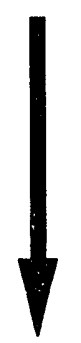

Bring sample to $300 \mathrm{~K}$. Lower pressure to less than 23 kbar.
Sample is Carriers trapped at DX transparent. centers. No free carrièr absorption.
Sample turns opaque.

DX centers photoionized. Free carrier absorption due to carriers in conduction band. Carriers have insufficient energy to overcome capture barrier into DX state.

Sample is opaque.
Shallow state is most stable form of donor. Free carrier absorption due to carriers in conduction band.

Fig. 4.16. The behavior of the samples studied is easily explained if DX centers are present. 
been assigned to ${ }^{28} \mathrm{Si}_{\mathrm{As}},{ }^{28} \mathrm{Si}_{\mathrm{Ga}}-{ }^{28} \mathrm{Si}$ As, $\mathrm{Si}-\mathrm{X}, \mathrm{Si}-\mathrm{Y},{ }^{29} \mathrm{Si}_{\mathrm{As}}$, and ${ }^{30} \mathrm{Si}_{\mathrm{As}}$. We exclude the possibility that the lower frequency peak we observe is due to any of the defects listed above based on the results of the following experiment. A bulk piece of Sample 11 was investigated by conventional absorption spectroscopy at zero pressure, and the only observable peak was that due to ${ }^{28} \mathrm{Siga}$. This is consistent with the fact that only one peak was observed when Sample 11 was in the DAC, but provides a more stringent test since the signalto-noise ratio for conventional spectroscopy is roughly a factor of twenty better than for spectroscopy performed in the DAC. As an additional check, we extrapolated the frequency of the new peak to zero pressure. It would be found at $376 \pm 1.5 \mathrm{~cm}^{-1}$. This value lies between the frequencies of the ${ }^{29} \mathrm{Si}$ and $30 \mathrm{Si}$ LVM peaks reported in Ref. 38, and the absorbance in these peaks is less than 10 percent of the absorbance due to ${ }^{28} \mathrm{Si}_{\mathrm{Ga}}$. In contrast, the new LVM peak observed has roughly half the absorbance of the ${ }^{28} \mathrm{Si}_{\mathrm{Ga}} \mathrm{LVM}$ peak. Finally, this technique is not sensitive to defect concentrations much below $6 \times 10^{17} \mathrm{~cm}^{-3}$, and it is unlikely that the three unirradiated samples we studied would have a defect besides $\mathrm{Si}$ at this high a concentration. The above arguments demonstrate conclusively that the lower frequency LVM is due to a previously unobserved defect related to Si. Since this defect is only formed by the application of pressure in a sample where free electrons are available, this new peak is identified as an LVM of the Si DX center.

This spectroscopic data can now be combined with Hall effect and resistivity data to allow us to infer the charge state of the DX center. Correcting for the different thicknesses of the sample, the area in the LVM absorption peak of sample 11 was compared with the sum of the areas of the SiGa and SiDX peaks of sample $1 U$ for several spectra and found to be equal to within thirty percent. Since the concentration of the Si impurities is the same in the two 
samples, this implies that the absorption cross-section for these two defects is the same to within this accuracy. This in turn implies that the ratio of the area in the LVM absorption peaks $A_{S i G a} / A_{S i D x}$ observed for a given sample is equal to $n_{S G_{a}} / n_{S i D X}$ to within thirty percent, where $n$ is the concentration of the corresponding defect.

The theoretical ratio $\mathrm{nSiGa} / \mathrm{n}_{\mathrm{SiDX}}$ depends on the charge state which is assumed for the DX center and the compensation ratio in the sample. The compensation ratio is defined as $n_{A} / n_{D}$, where $n_{A}$ is the concentration of minority acceptors and $n_{D}$ is the concentration of majority donors. If the $D X$ center were a neutral defect, all uncompensated donors should undergo the DX transformation and the ratio of the concentration of Si shallow donors to Si DX centers would be

$$
\frac{n_{S i_{G a}}}{n_{S i_{D X}}}=\frac{n_{a}}{n_{d}-n_{a}}=\frac{\theta}{1-\theta} .
$$

If the DX center were negatively charged, only one half of the uncompensated donors can transform into DX centers, since one half of the Si atoms would need an additional electron from the other half. The concentrations would then be related to the compensation ratio by

$$
\frac{n_{S i G a}}{n_{S i D X}}=\frac{\frac{1}{2}\left(n_{d}-n_{a}\right)+n_{a}}{\frac{1}{2}\left(n_{d}-n_{a}\right)}=\frac{1+\theta}{1-\theta} .
$$

The compensation ratio can be determined from the mobility, $\mu$, and the free carrier concentration, $n$, as shown below (Walukiewicz, 1990). In heavily doped samples, the main factor limiting mobility is ionized impurity scattering. 
Taking only this scattering mechanism into account, the following relationship between compensation ratio, free carrier concentration, and mobility can be derived:

$$
\theta=\frac{n_{a}}{n_{d}}=\frac{1-y}{1+y}
$$

where

$$
\begin{aligned}
& y=\mu\left[\frac{2 e^{3}\left(m^{*}\right)^{2} F_{c c}}{3 \pi \varepsilon^{2} h^{3} n}\right], \\
& F_{C C}=\ln (1+\zeta)-\frac{\zeta}{1+\zeta^{\prime}}
\end{aligned}
$$

and

$$
\zeta=\frac{(3 \pi n)^{1 / 3} h^{2} \varepsilon_{0}}{e^{2} m^{*}}
$$

Here $\varepsilon_{0}$ is the dielectric constant in the material, $n=n_{d}-n_{a}$, $e$ is the charge on the electron, and $\mathrm{m}^{*}$ is the effective mass of the electron. The concentration and mobility can be obtained from a combination of Hall effect and resistivity analysis, and this was done for all the unirradiated samples which have been examined in this study. The results are given in Table 4.2. The analysis took into account the concentration dependence of the effective mass (Raymond, 1979).

We combine the Hall effect analysis with our spectroscopic data, and plot the results in Figure 4.17 along with curves for the predictions of the DX- and DX0 models. Our results clearly support the negative-U model for the DX center. 
Table 4.2. Results from Hall effect and resistivity analysis

$\begin{array}{llll}\text { Sample } & \underline{N_{d}-N_{a}\left(10^{18} \mathrm{~cm}^{-3}\right)} & \mu_{110 K}\left(10^{3} \mathrm{~cm}^{2} \mathrm{Ns}\right) & \theta \\ 11 & 0.63 & \ldots & \ldots \\ 1 \mathrm{U} & 0.63 & 2.70 & 0.31 \\ 2 & 2.9 & 1.53 & 0.39 \\ 3 & 2.1 & 1.41 & 0.47\end{array}$




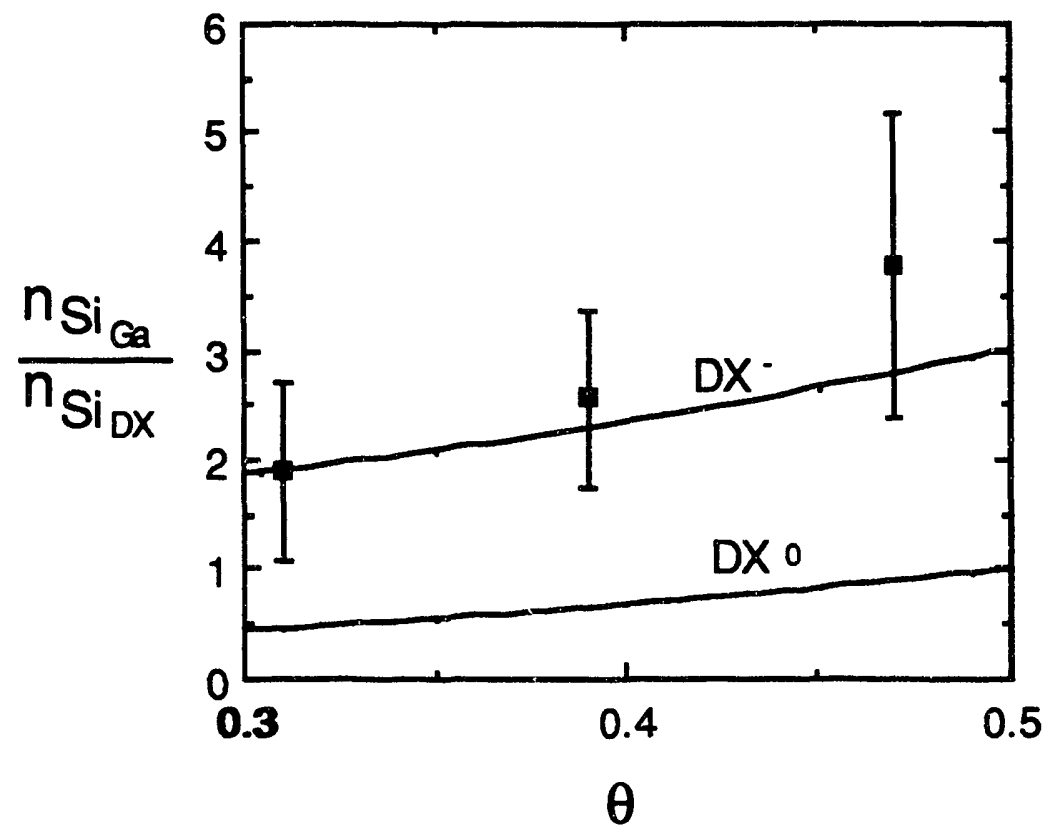

Fig. 4.17. Comparison between experimental results and theoretical predictions for the ratio $\mathrm{nSiGa}^{\text {nSiDX }}$ as a function of compensation ratio. 
Since there is not complete agreement between different sources on obtaining compensation ratios from concentration and mobility data, it is important to consider how using theories other than the one chosen here would affect the interpretation of the data. Any model which includes scattering effects in addition to ioinzed impurity scattering would lead to lower compensation ratios, and would therefore shift the data points even further away form the DXO curve sh. Jwn in Figure 4.17. A recent model (Meyer, 1991) which claims that screening effects in other models have been overestimated also leads to lower compensation ratios. It therefore appears that using other models would not change our conclusion that the DX center is negatively charged.

\subsection{Comparison Between Experiment and Theon}

Since this experiment was performed, there have been two calculations of the LVM frequencies of the DX center and one calculation of the pressure dependence of these frequencies. A recent calculation by Saito et. al (1992) is in fairly good agreement with this experiment. They calculated the energies of several different large relaxation geometries and found that the state with the lowest energy was essentially that suggested by Chadi and Chang (1988), with the exception that they predicted a Si donor would move $1.38 \AA$ in the (111) cirection rather than $1.17 \AA$. The bonding angle of the $\mathrm{Si}$ atom is $115^{\circ}$, which is intermediate between $s p^{2}$ and $s p^{3}$ bonding. In this model, the nearest neighbor bond distance of the $\mathrm{Si}$ atom changes form $2.43 \AA$ to $2.42 \AA$ upon transformation, which is consistent with the EXAFS data discussed in Section

\subsection{3.}


They also calculated frequencies of the LVM for this lattice relaxed state. As expected, there are two vibrational modes, a singly degenerate $a_{1}$ mode in the (111) direction and a doubly degenerate e mode. These vibrations are shown in Figure 4.18. The zero pressure frequencies are listed in Table 4.3 along with the experimentally measured (and extrapolated) frequencies from this thesis. If the $a_{1}$ mode is at the frequency calculated by Saito et al, then it would fall within the restrahlen band of GaAs, where the sample is completely reflective and would thus be unobservable. (The restrahlen band is the region between the longitudinal optic and transverse optic phonon frequency, where the dielectric constant is negative and the sample is therefore completely reflective. It will be discussed in detail in Chapter 5). They calculate that the e mode should be at $392 \mathrm{~cm}^{-1}$ whereas the extrapolation of the observed DX mode frequency is $376 \mathrm{~cm}^{-1}$. Since these calculations usually are only accurate to within $10 \%$, this is reasonable agreement.

Although these calculations are extremely computer intensive, Saito et al give a rough physical argument for why the triplet substitutional $\left(T_{d}\right)$ mode has essentially the same frequency as the e mode. Since bond bending forces are much weaker than bond stretching forces in tetrahedrally bonded materials, only the Si-As stretching bonds are considered. In this approximation, the ratio of the two frequencies is given by a valence force field (VFF) model to be (Herzberg, 1945)

$$
R(V F F)=\frac{v\left(T_{d}\right)}{v(e)}=\left[\frac{k^{\prime}\left(1+\frac{4}{3} M\right)}{k^{\prime}\left(1+\frac{3}{2} M \sin ^{2} \beta\right)}\right]^{\frac{1}{2}},
$$




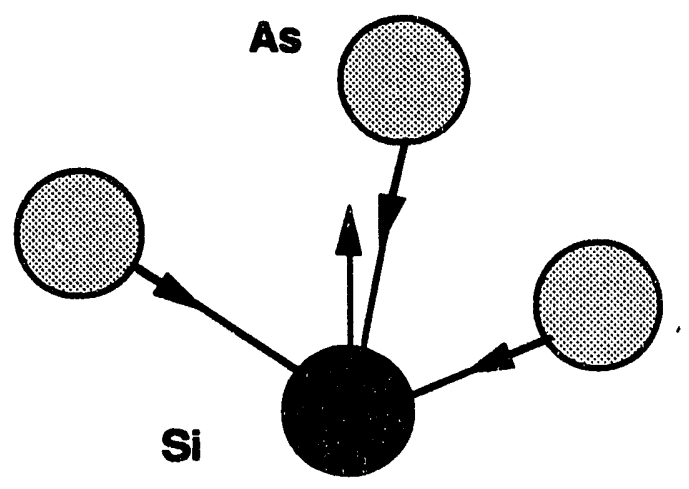

$a_{1}$ vibration

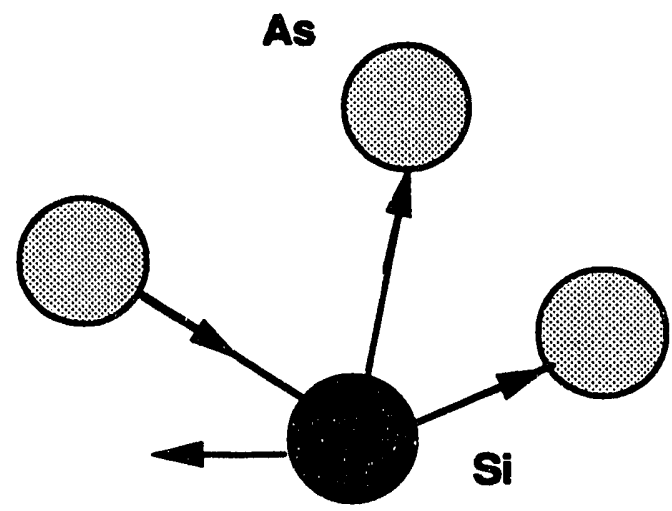

e vibration

4.18. Local vibrational modes for the DX center in the broken bond geometry. (Saito, 1992). 
Table 4.3. Comparison of experimental and theoretical frequencies of Si DX LVMs $\left(B B=\right.$ broken bond configuration, $A_{1}=A_{1}$ state configuration, sub $=$ substitutional position) (Cheong and Chang, 1992).

\begin{tabular}{|c|c|c|c|}
\hline $\begin{array}{l}\text { Mode } \\
a_{1} \text { (BB) }\end{array}$ & $\begin{array}{l}\text { Experiment } \\
\text { not observed }\end{array}$ & $\begin{array}{l}\text { Saite Calculation } \\
\text { in restrahlen band }\end{array}$ & $\begin{array}{l}\text { Jones Calculation } \\
\text { in restrahlen band }\end{array}$ \\
\hline e (BB) & $376 \mathrm{~cm}^{-1 *}$ & $392 \mathrm{~cm}^{-1}$ & $432 \mathrm{~cm}^{-1}$ \\
\hline triplet $\left(\mathbf{A}_{1}\right)$ & $376 \mathrm{~cm}^{-1^{*}}$ & not calculated & 347 \\
\hline triplet (sub) & $384 \mathrm{~cm}^{-1}$ & $370 \mathrm{~cm}^{-1}$ & $386 \mathrm{~cm}^{-1}$ \\
\hline
\end{tabular}


where $k$ and $k$ are the force constants for the substitutional and broken-bond geometries, $\mathrm{M}$ is the ratio in the atomic mass between the $\mathrm{As}$ and $\mathrm{Si}$ atom, and $\beta$ is the angle between the [111] axis and the Si-As bond in the broken-bond geometry. For the defect geometry proposed by Saito et al, assuming $k=k$ ' since there is almost no change in the bond length before and after $R(V F F)=0.98$. Their more rigorous calculations give $R(V F F)=0.94$, while the experimental value is 1.02 . They claim that the agreement between experiment and theory would be even better if relaxations of the next-nearest neighbor $\mathrm{Ga}$ atoms were taken into consideration, since this would lengthen the Si-As bonds somewhat and lower the $a_{1}$ LVM frequency. Further movement of the $\mathrm{Si}$ atom should give the same effect. It would therefore be interesting for them to carry out a calculation of the dependence of the LVM frequencies of the modes as a function of the position of the Si atom. If the Si atom had to move significantly in order for theory and experiment to agree, than its position would be in conflict with the EXAFS data of Hayes et al and Kitano et al, which provides an independent criterion. In summary, Saito et als calculation is consistent with the conclusion of the work in this thesis that the DX center is negatively charged and is in reasonable agreement with the observed frequency of the LVMs.

Another recent calculation was performed by Jones and Öberg (1992). They calculated the energetic position of both the $A_{1}$ small lattice relaxation state as well as the energy of the Chadi and Chang broken bond state. The results of their calculations of the LVM frequencies for these defect states are shown in Table 4.3 next to the results of Saito. They calculate a smaller relaxation than Saito for the broken bond configuration, and their estimation of the e mode is therefore $11 \%$ higher than that of Saito. It is not clear whether they would calculate the same frequencies for the same geometry. Jones and Öberg also claim that the absorption coefficient of this defect is relatively weak. 
On the basis of these claims they reject the ioentification of the newly discovered mode as being due to a vibration of the broken-bond geometry. They calculate that the frequency of the small lattice relaxation $A_{1}$ vibration (which is just the Si atom vibrating in the tetrahedron formed by the relaxed As atoms) would be $347 \mathrm{~cm}^{-1}$ and that it would have an absorption coefficient ten times greater than that of the broken-bond e mode. They therefore feel that this should be identified with the new LVM observed in this experiment. There are two points which this interpretation fails to address, however. The first point is that it ignores the conclusion that the DX center is negatively charged. The second point is that this interpretation fails to explain the occurrence of persistent photoconductivity. Jones et al suggested that the broken bond geometry and $A_{1}$ state coexist, and that the broken-bond state is responsible for the persistent photoconductivity. However, if the $A_{1}$ state exists, there should be no barrier for an electron to fall into it. Therefore, as soon as a broken-bond center was optically ionized, it should fall into an $A_{1}$ state and there should be no persistent photoconductivity. This contradicts what was observed in our experiment.

Another calculation has recently been made by Chadi (1992b) in which he claims that the $A_{1}$ state should be also be negatively charged. He also claims this defect would exhibit persistent photoconductivity. The properties of such a defect would therefore also be consistent with the findings of this research. However, Chadi calculates that the $A_{1}$ state is $0.5 \mathrm{eV}$ higher than that of the broken-bond geometry in GaAs:Si, and so this is not a likely candidate.

Finally, a new calculation by Cheong and Chang (1992) investigated the pressure derivatives of the LVMs of both the broken bond configuration and the $A_{1}$ state. The results are summarized in Table 4.4. They show that the pressure derivatives calculated for the DX broken bond configuration and shallow donor 
substitutional LVM frequencies are in rough agreement with those observed. In contrast they find that the pressure derivative for the $A_{1}$ geometry LVM frequency is a factor of two larger than the observed values. This provides further confirmation for the broken bond model of Chadi and Chang. It is important to point out that the pressure dependences of the substitutional mocie is predicted to be higher than that of the e mode, which is not what is observed. It would again be interesting to see how these pressure derivatives depend on the position of the Si atom, and whether further movement of the Si atom would bring about closer agreement between experiment and theory.

In summary, an LVM of the Si DX center in GaAs has been observed for the first time. The shift of the LVM frequency with pressure was found to be $d v_{\text {SiDX }} / \mathrm{dp}=0.61 \pm 0.04 \mathrm{~cm}^{-1} / \mathrm{kbar}$ for the Si DX center and $d v_{S i G a} / \mathrm{dp}=0.66 \pm$ $0.03 \mathrm{~cm}^{-1} / \mathrm{kbar}$ for $\mathrm{SiGa}_{\mathrm{Ga}}$. The ratio of the area of the $\mathrm{Si}_{\mathrm{Ga}}$ absorption peak to that of the Si DX absorption peak has been combined with Hall effect and resistivity analysis to provide further evidence supporting the negative- $U$ model for the $D X$ center. Two calculations have been made for the LVM frequencies of the DX center, but only the calculation of Saito et al appears to be in agreement with all of the experimental results presented in this chapter. This supports the broken bond configuration model for the DX center. Further support for this model comes from the calculations of Cheong and Chang which show that the pressure derivatives for the LVM frequencies observed experimentally agree with those predicted for the broken bond configuration but are in conflict with those calculated for the $A_{1}$ breathing mode relaxation. 
Table 4.4. Comparison of experimental and theoretical pressure derivatives of the local vibrational mode frequencies of Si $D X$ centers $(B B=$ broken bond configuration, $A_{1}=A_{1}$ state configuration, sub $=$ substitutional position) (Cheong and Chang, 1992).
Mode
Experiment
Calculation
e (BB)
$0.61 \mathrm{~cm}^{-1} / \mathrm{kbar}^{*}$
$0.71 \mathrm{~cm}^{-1} / \mathrm{kbar}$
triplet $\left(\mathbf{A}_{1}\right)$
$1.27 \mathrm{~cm}^{-1} / \mathrm{kbar}$
triplet (sub)
$0.66 \mathrm{~cm}^{-1} / \mathrm{kbart}^{*}$
$0.53 \mathrm{~cm}^{-1} / \mathrm{kbar}$

*Assignments suggested by Cheong and Chang 


\section{Formation of a DX Center in InP Under Hydrostatic Pressure}

\subsection{Motivation for the Experiment}

It was mentioned in Chapter 1 that DX centers have been observed in GaAs under hydrostatic pressure (Mizuta, 1985) and AlGaAs (Lang, 1977). Deep levels with DX-like properties have also been observed in various alloys of GaAsP (Craford, 1968), InGaP, InGaAIP, InAlAs, and AlGaSb (Mooney, 1990). This leads to the question of whether the existence of such defects is in fact a general feature of all III-V semiconducting compounds. If this were the case, one must determine why these states are not observed in every III-V semiconductor and whether or not it is possible to predict under what circumstances they will appear. It was with these questions in mind that the experiment described in this chapter, a search for the formation of DX centers in InP under hydrostatic pressure, was performed.

In making an educated guess as to whether or not DX centers will form when hydrostatic pressure is applied to a given III-V semiconductor, there are several characteristics of the material which must be considered. Since it was pointed out in Section 1.2 that the formation of DX centers is caused by changes in the structure of the conduction band, a logical choice of material is one which has a conduction band structure and band minima pressure derivatives which are similar to those of GaAs. Many III-V semiconductors can be eliminated from consideration by virtue of the fact that they are indirect band gap semiconductors with a conduction band minimum at $X$. The $X$ minimum will move down in energy much faster than the average conduction band (see arguments in Section 4.2.1.1). This implies that if the $X$ band donors are the 
most stable form of the donor at zero pressure, then they will remain lower in energy than a DX state at all pressures. BN, BAs, AIP, AIAs, AISb and GaP all have their conduction band minimum at $X$. Since the DX center wavefunction is made up of all the conduction band states, it is also important that the energies of the other minima are not too far above the $\Gamma$ minimum. Otherwise the state will be very high in energy and it will probably require extremely high pressures to bring the state into the gap. A related point is that the material should not undergo a phase transition at a pressure which is lower than the pressure at which the DX center becomes the most stable form of the donor. In InAs, the energy separations between the $\Gamma, X$, and $L$ conduction band minima are $E(X)$ $E(\Gamma)=1.91 \mathrm{eV}$ and $E(L)-E(\Gamma)=1.16 \mathrm{eV}$ (Landolt, 1987), much larger than those in GaAs. This implies it would take a large pressure to bring a DX state (assuming it existed) into the gap. However, InAs undergoes a phase transition at $70 \mathrm{kbar}$ (Landolt, 1987).

Taking the above arguments into account we find that $\ln P$ is a strong candidate among the III-V semiconductors in which to attempt to form DX centers. The pertinent parameters of this material are listed in Table 5.1 along with those of GaAs. The table shows that the energy separation between the $\Gamma$ conduction band minimum (CBM) and the $X$ and L CBMs in InP is roughly twice that found in GaAs. Since the band edges are higher, one must be concerned with the phase transition pressures. For InP, though, it will turn out that the DX center transition pressure is in fact below the phase transition pressure. We have omitted GaSb from consideration, even though the energy separations between $\Gamma$ and $L$ and $X$ are small, because it is difficult to obtain high quality $n$ type material. Most GaSb is strongly p-type due to native defects. However, as will be discussed later, deep donors exhibiting DX-like behavior have been observed in this material more than a decade ago (Dmowski, 1979). 
Table 5.1. - Comparison of Band Structures of GaAs and InP

$\begin{array}{lll} & \text { GaAs } & \text { InP } \\ E_{X}-E_{\Gamma}(\mathrm{eV}) & 0.4(\mathrm{a}) & 0.96(\mathrm{c}) \\ E_{L}-E_{\Gamma}(\mathrm{eV}) & 0.2^{(a)} & 0.40(\mathrm{c}) \\ \mathrm{d} E_{\Gamma} / \mathrm{dp}(\mathrm{meV} / \mathrm{kbar}) & 12 \pm 0.5(\mathrm{a}) & 8.4 \pm 0.5(\mathrm{~d}) \\ \mathrm{d} E_{X} / \mathrm{dp}(\mathrm{meV} / \mathrm{kbar}) & -1.6 \pm 1.0(\mathrm{a}) & -2.0 \pm 1.0(\mathrm{e}) \\ \mathrm{d} E_{\mathrm{L}} / \mathrm{dp}(\mathrm{meV} / \mathrm{kbar}) & 5.5 \pm 1.0(\mathrm{a}) & 3.7 \pm 1.0(\mathrm{f}) \\ \text { Pphase transition (kbar) } & 145^{(\mathrm{b})} & 110(\mathrm{f})\end{array}$
(a) (Chadi, 1989)
(b) (Zhang, 1989)
(c) (Landolt, 1987)
(d) (Tozer, 1988)
(e) (Chang, 1984)
(f) (Goni, 1989) 
The remainder of this chapter will describe the discovery of a DX center in InP:S under hydrostatic pressure greater than 82 kbar (Wolk, 1992). This defect exhibits the persistent photoconductivity typical of such centers. The optical ionization energy for this new DX center is between 0.86 and $1.02 \mathrm{eV}$, and we have measured the energy dependence of the optical absorption cross section. The thermal barrier for capture from the shallow donor state into the deep DX state is in the range $0.23-0.33 \mathrm{eV}$. At zero pressure the energy of the DX center is estimated to lie $0.51 \pm 0.07 \mathrm{eV}$ above the $\Gamma$ conduction band minimum. Using this last result, we will suggest a method for predicting the conditions under which the DX center will be the most stable form of the donor in any III-V semiconductor. It will turn out that the preceding discussion did not consider one of the most important factors in determining whether or not DX centers will be observable in a given semiconductor, which is the energy separation between the conduction band minimum and the vacuum level.

\subsection{Determination of the DX Center. Transition Pressure}

\subsubsection{The Restrahlen Band}

Determining the pressure at which the $\mathrm{S}$ donor in $\mathrm{InP}$ transforms into a DX center involves the observation of the InP restrahlen band, which is a region of high reflectivity of the crystal between the transverse optic (TO) and longitudinal optic (LO) phonon frequencies. The explanation for the origir of this feature arises very naturally as part of a more general discussion of the interaction between a transverse optic phonon and a photor. The guantum of 
the coupled phonon-photon field is called a polariton. We begin by considering Maxwell's equations in a non-magnetic material.

$$
\begin{aligned}
& \nabla \cdot D=4 \pi \rho, \\
& \nabla \cdot H=0, \\
& \nabla \times E=-\frac{1 \partial H}{C} \partial t \\
& \nabla \times H=\frac{1 \partial D}{C \frac{\partial}{}},
\end{aligned}
$$

where $\mathbf{D}$ is the electric field displacement, $\mathbf{E}$ is the electric field, $\mathbf{H}$ is the magnetic field, $\rho$ is the charge density, $c$ is the speed of light, and $t$ is time. In a long wavelength ( $k=2 \pi / \lambda \approx 0$ ) optical mode in an ionic crystal, the positive and negative ions move in opposite directions, creating a polarization density P. D and $E$ are related through the polarization by

$$
D=\varepsilon E=E+4 \pi P,
$$

where $\varepsilon$ is the dielectric constant. In the absence of free charge

$$
\nabla \cdot D=0 .
$$

If we ignore the time dependence of the magnetic fields, then Eq. 5.3 becomes

$$
\nabla \times E=0 .
$$


In a cubic crystal $\boldsymbol{\varepsilon}$ is a scalar and $\mathbf{D}, \mathbf{E}$, and $\mathbf{P}$ are all parallel to one another. If they all have the same spatial dependence we can write

$$
\left\{\begin{array}{l}
D \\
E \\
\mathbf{P}
\end{array}\right\}=\operatorname{Re}\left\{\begin{array}{l}
D_{0} \\
E_{0} \\
P_{0}
\end{array}\right\} e^{i k \cdot r}
$$

Eq. 5.6 becomes

$$
k \cdot D_{0}=0
$$

which implies that either

$$
\mathbf{D}=\mathbf{0} \text { or } \mathbf{D}, \mathbf{E} \text {, and } \mathbf{P} \text { are } \perp \mathbf{k} \text {. }
$$

Eq. 5.7 becomes

$$
k \times E_{0}=0,
$$

which means that either

$$
E=0 \text { or } \mathbf{D}, \mathbf{E} \text {, and } \mathbf{P} \text { are } \| \mathbf{k} \text {. }
$$

For an LO mode $\mathbf{P}$ is parallel to $k$, so this implies that $\mathbf{D}=0$. From Eq. 5.5 this requires that

$$
E=-4 \pi P, \varepsilon=0 \quad \text { (for LO mode). }
$$


For a TO mode $P$ is perpendicular to $k$, so Eq. 5.11 implies that $E=0$. From Eq. 5.5 this means that

$$
E=0, \varepsilon=\infty \quad \text { (for TO mode). }
$$

The zero of the dielectric constant can therefore be identified with the LO mode frequency while the TO mode occurs at the pole of the dielecric constant.

We now consider the effect of the the time dependence of the magretic field in the right hand side of Eq. 5.3. We iook for a solution to Maxwall's equations of the form $\left.E=E_{0} e^{i(k \cdot r-x i}\right)$. Taking the curl of Eq. 5.3 and combining it with Eq. 5.4 gives

$$
\nabla \times(\nabla \times E)=-\nabla^{2} E=-k^{2} E=\frac{i \omega}{c} \nabla \times H=\frac{-\omega^{2}}{c^{2}} D .
$$

This is a wave equation and it can be written in the form

$$
c^{2} k^{2} E=\omega^{2} \varepsilon E,
$$

which leads to

$$
\varepsilon(\omega)=c^{2} k^{2} / \omega^{2} .
$$

Ignoring the time dependence of the magnetic field in the right hand side of Eq. 5.3 led to the conclusion that $\varepsilon=\infty$ for a transverse optic phonon. This approximation is therefore reasonable so long as ck $>\omega$. The frequency of 
optic phonons is roughly $\omega_{D}=k, s$, where $s$ is the speed of sound and the $\omega_{D}$ and $K_{D}$ are the Debye wavenumber and frequency, respectively. The Debye frequency is the cutoff frequency for phonon propagation in the solid. The validity of the approximation therefore requires that

$$
\left(k k_{d}\right) \ll s / c
$$

Since $k_{D}$ is comparable to the dimensions of the Brillouin zone and $s / c$ is approximately $10^{-5}$, the approximation is valid for the whole Brillouin zone except for $k$ very close to zero. However, this is exactly the part of the Brillouin zone we are concerned with when considering the interaction of the TO phonons with a photon, so it is clear that we cannot ignore the right side of Eq.5.3.

We now describe the coupling of the TO phonon to an electromagnetic wave. The first step is to rewrite Eq. 5.15 as

$$
-c^{2} k^{2} E=\omega^{2}(E+4 \pi P)
$$

The polarization $\mathbf{P}$ caused by the electromagnetic wave is proportional to the displacement of the positive ions relative to the negative ones. We take the motions of these ions to be harmonic-oscillator like, so the equation of motion for $P$ is also of this form. The equation of motion for an undamped, forced harmonic oscillator is given by

$$
\frac{d^{2} x}{d t^{2}}+\omega_{0}^{2} x=\frac{F}{m}
$$


where $F$ is the force driving the oscillation, $m$ is the mass of the oscillator, and $\omega_{0}$ is the natural frequency of the oscillator. The equation for $P$ can thus be written as

$$
-\omega^{2} P+\omega^{2} T O P=\left(N q^{2} / M\right) E,
$$

where there are $\mathbf{N}$ ions pairs of effective charge $q$ and reduced mass $M$ per unit volume. Equations 5.18 and 5.20 can only be solved simultaneously if

$$
\left|\begin{array}{cc}
\omega^{2}-c^{2} k^{2} & 4 \pi \omega^{2} \\
N q^{2} / m & \omega^{2}-\omega^{2} \circ
\end{array}\right|=0 .
$$

The solution to this coupled set of equations gives the dispersion relation plotted in Figure 5.1. Two solutions exist at $k=0$ (Kittel, 1976), namely:

$$
\omega^{2}=0 \text {, }
$$

which is a photon-like solution since $\omega=c k$ in this region, and

$$
\omega^{2}=\omega^{2} \mathrm{TO}+4 \pi\left(N q^{2} / \mathrm{M}\right)=\omega^{2} \mathrm{LO}
$$

which is a polariton, since here the photon and phonon are mixed. This latter $\omega^{2}$ can be identified as $\omega^{2}$ Lo because we will see in a momen' that it once again defines the zero of the dielectric function. From Eq. 5.20 we have

$$
\varepsilon(\omega)=1+4 \pi P / E=1+\frac{4 \mathrm{Ne}^{2} / \mathrm{M}}{\omega_{10}^{2}-\omega^{2}} .
$$




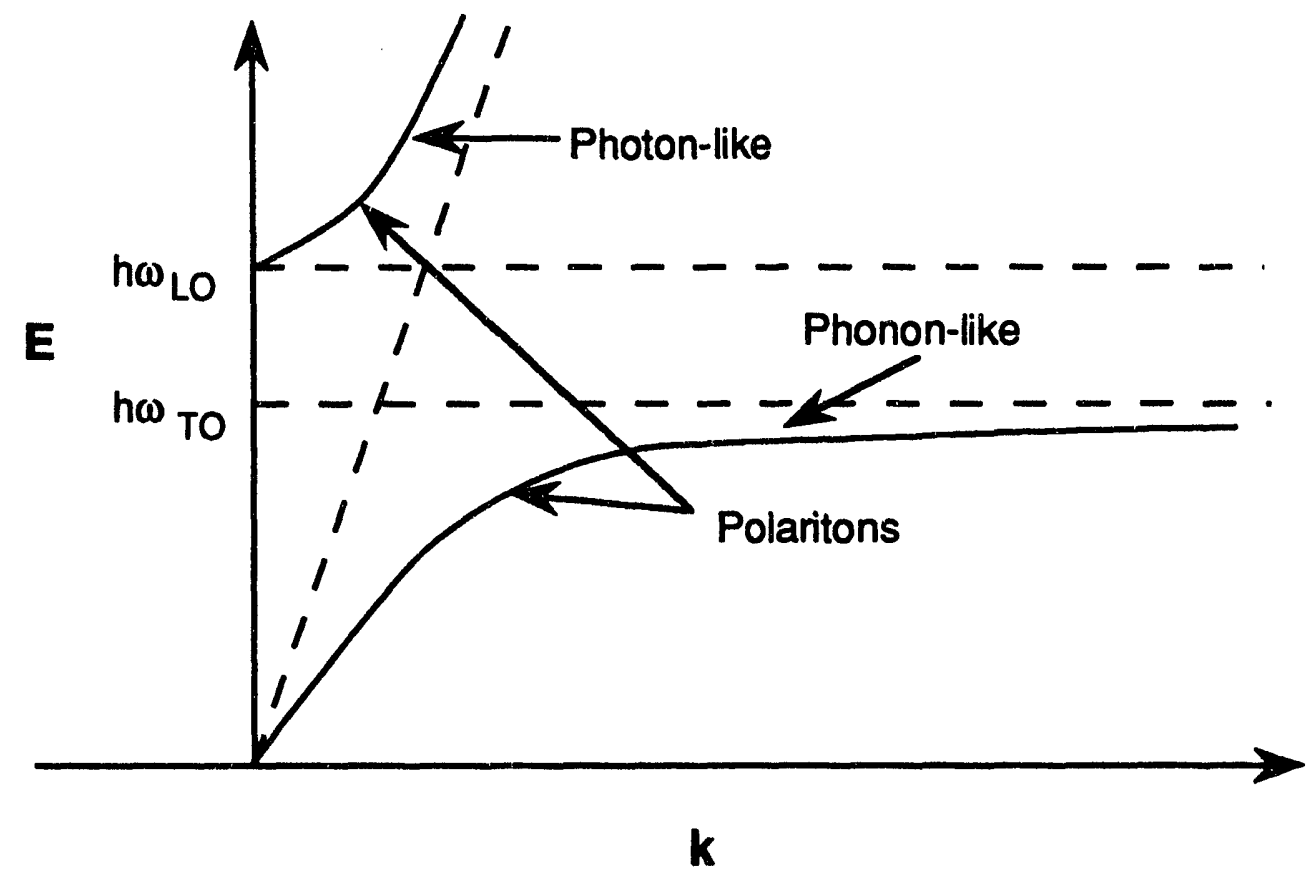

Fig. 5.1. Dispersion relationship for coupled transverse optic phonon-photon field. Regions where behavior of modes are photon, phonon, or polariton like are indicated. 
The contribution to the polarization from the ionic cores can be taken into account through the infinite frequency cielectric constant $\varepsilon(\infty)$, requiring Eq 5.24 to be rewritten as

$$
\varepsilon(\omega)=\varepsilon(\infty)+\frac{4 \mathrm{Ne}^{2} / \mathrm{M}}{\omega_{\text {fo }}^{2}-\omega^{2}}
$$

Combining Eqs. 5.23 and 5.25 gives

$$
\varepsilon(\omega)=\varepsilon(\infty)\left(\frac{\omega_{10}^{2}-\omega^{2}}{\omega_{T O}^{2}-\omega^{2}}\right)
$$

A plot of $\varepsilon(\omega)$ is shown in Figure 5.2 and it can be seen that it is negative between $\omega$ TO and $\omega_{\text {LOO }}$. From Eq. 5.16 it is obvious that a negative dielectric constant implies that $k$ or $\omega$ must be imaginary and that all electromagnetic waves in this frequency region will be damped. The reflectivity of the crystal is very high in this region, causing the sample to appear to be totally absorbant in this frequency range in an absorption experiment. This region of high reflectivity is called the restrahlen region. Restrahl is the German word for "remaining ray". This frequency band was given that name because if a light beam was reflected between two plates of an ionic crystal then the only radiation remaining after multiple reflections would be that which fell in the restrahlen band.

\subsubsection{Determining the Shallow-to-Deep Transition Pressure}

I will now discuss the technique for finding the shallow-to-deep donor transition pressure. The samples we used were $\operatorname{InP}: S$ with a free carrier concentration $\mathrm{n}=2.1 \times 10^{18} \mathrm{~cm}^{-3}$. Hydrostatic pressure was applied to the 


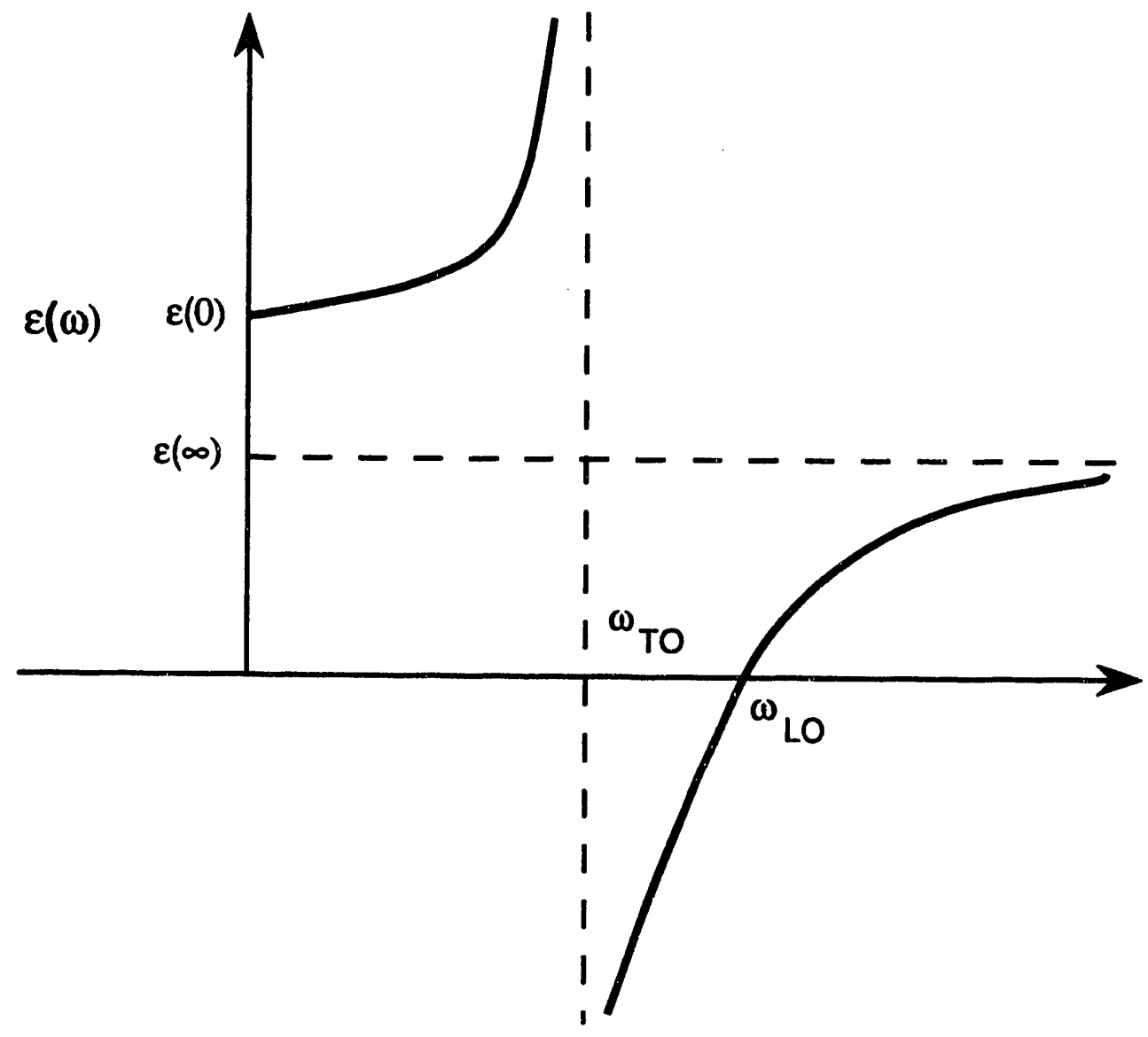

Fig. 5.2. Dielectric constant, $\varepsilon$, as a function of frequency, $\omega$. It is negaitve in the restrahlen region. 
samples using the diamond anvil cell described in Chapter 2 with liquid nitrogen as the pressure medium. Fourier transform spectroscopy was then performed using the techniques also described in Chapter 2 (Wolk, 1992b). All spectra were taken at $\mathrm{T}=5 \mathrm{~K}$.

When the sample is at low pressure, the $S$ donor electrons occupy the shallow donor state. At this high doping level, however, the shallow impurity band has broadened so much that it merges with the conduction band. This makes the sample opaque to far infrared radiation because of free carrier absorption (see Section 4.3). When the sample is put under a sufficiently large hydrostatic pressure, though, the electrons are trapped at the deep DX levels and the sample becomes transparent in the far infrared. Therefore, the transition pressure is the pressure at which the sample becomes transparent. We observe an infrared signal even below the transition pressure because of light leaking around the sample, but because the sample is opaque we see no InP related features. Above the DX formation pressure we detect light passing through as well as around the sample. In this case we very clearly see the restrahlen band of InP. Spectra above and below the formation pressure are shown in Figure 5.3. By looking for the appearance of the restrahlen band, we find that the pressure required for DX center formation in our samples is $82 \mathrm{kbar}$.

There are other explanations for the decrease in free carrier concentration, but we can rule them out as follows. The pressure-induced transparency is clearly not due to a crossing of the $\Gamma$ and $X$ conduction bands, resulting in deeper $X$-band donors, since the new deep state exhibits persistent photoconductivity. Additionally, the observed spectral behavior is reversible with pressure, so it cannot be attributed to permanent structural defects created by the high pressure. 


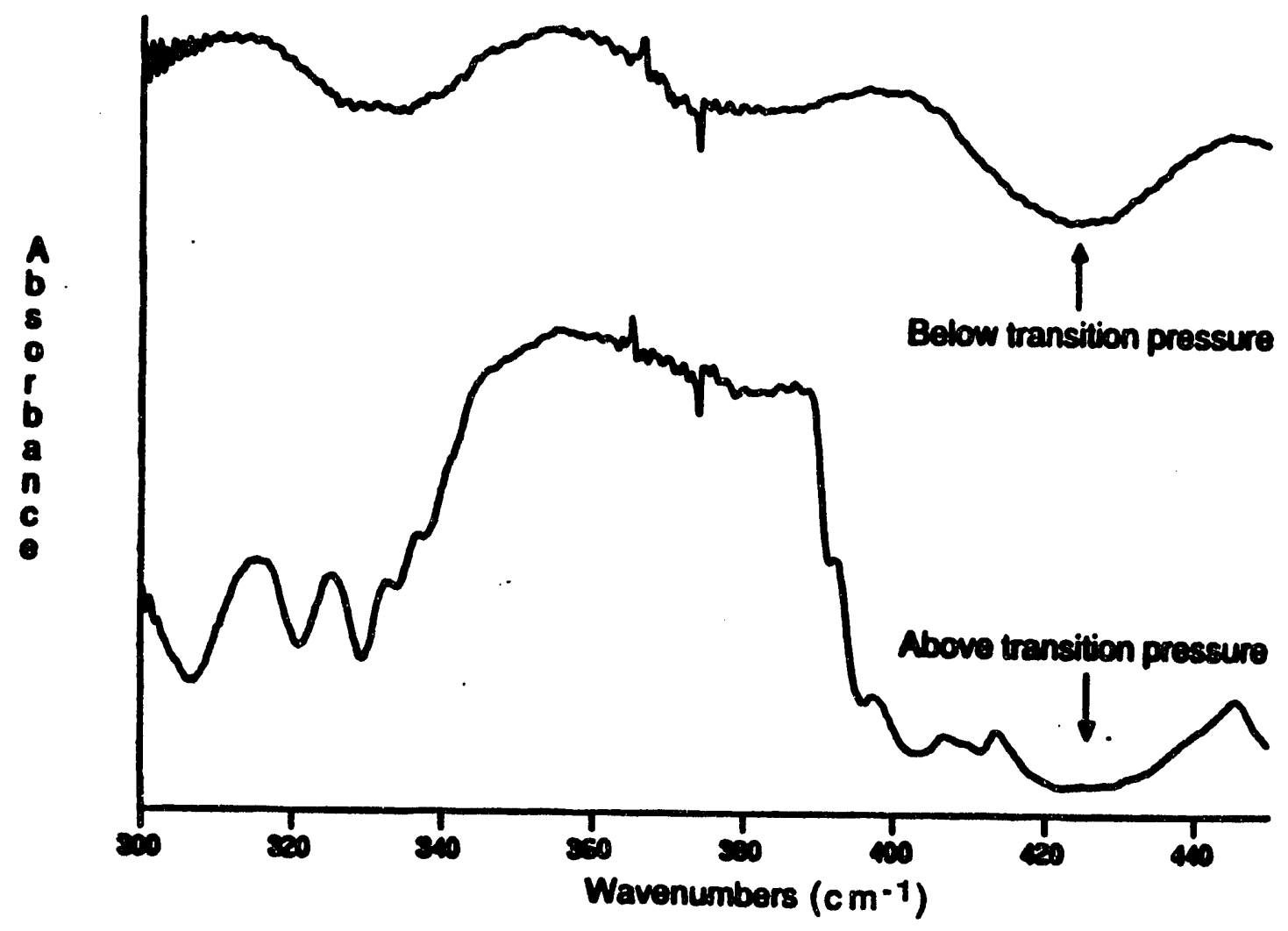

Fig 5.3. Spectra of inP:S above and below the shallow ronor-to-DX center transition pressure. 
The transition pressure can be used to estimate the energy of the DX level at zero pressure relative to the shallow donor level. Following the arguments of Chadi and Chang detailed in Section 4.2.1.1 for deep levels, the pressure derivative of the DX center level is the same as that of the conduction band averaged over all k-space. This average pressure derivative of the conduction band can be estimated using the expression (Chadi, 1989b)

$$
\frac{d E_{C B}}{d p}=\left(\frac{(d E(\Gamma)+3 d E(X)+4 d E(L)) / 8}{d p}\right)
$$

We use $\mathrm{dE}(\Gamma) / \mathrm{dp}=8.4 \pm 0.5 \mathrm{meV} / \mathrm{kbar}, \mathrm{dE}(X) / \mathrm{dp}=-2.0 \pm 1.0 \mathrm{meV} / \mathrm{kbar}$, and $\mathrm{dE}(\mathrm{L}) / \mathrm{dp}=3.7 \pm 1.0 \mathrm{meV}$ kbar with respect to the valence band maximum (Tozer, 1988; Chang, 1984; Goni, 1989). This gives $\mathrm{dE} \mathrm{CB}_{\mathrm{B}} \mathrm{dp}=2.2 \pm 0.6 \mathrm{meV} / \mathrm{kbar}$, which implies that the DX level approaches the conduction band minimum at $\Gamma$ at the rate $\mathrm{dE}(\Gamma) / \mathrm{dp}-\mathrm{dE} \mathrm{CB}_{\mathrm{B}} / \mathrm{dp}=6.2 \pm 0.8 \mathrm{meV} / \mathrm{kbar}$.

The restrahlen band can be observed when the free carrier absorption becomes sufficiently small. Using a relationship between the concentration and magnitude of free carrier absorption (Walukiewicz, 1980), it can be calculated that the sample is $90 \%$ transparent by the time the DX level lies $25 \mathrm{meV}$ below the shallow donor level. The DX level must therefore lie within $25 \mathrm{meV}$ of the conduction band minimum at 82 kbar. Taking this $25 \mathrm{meV}$ uncertainty into account, a transition pressure of $82 \mathrm{kbar}$ implies that the DX level is $510 \pm 70$ meV above the $\Gamma$ conduction band minimum at zero pressure.

It is interesting to note that, as shown in Figure 5.4, the difference between the energy at $P=0$ of the $S \mathrm{DX}$ center in $\operatorname{InP}(.510 \pm .07 \mathrm{eV})$ and GaAs (.150 eV) (Chadi, 1989b) is equal to the conduction band offset between InP and GaAs $(\approx 0.4 \mathrm{eV}$ ) (Nolte, 1988; Van de Walle,1989; Tersoff, 1985) within the 


\section{GaSb GaAs $\underline{\ln P}$}

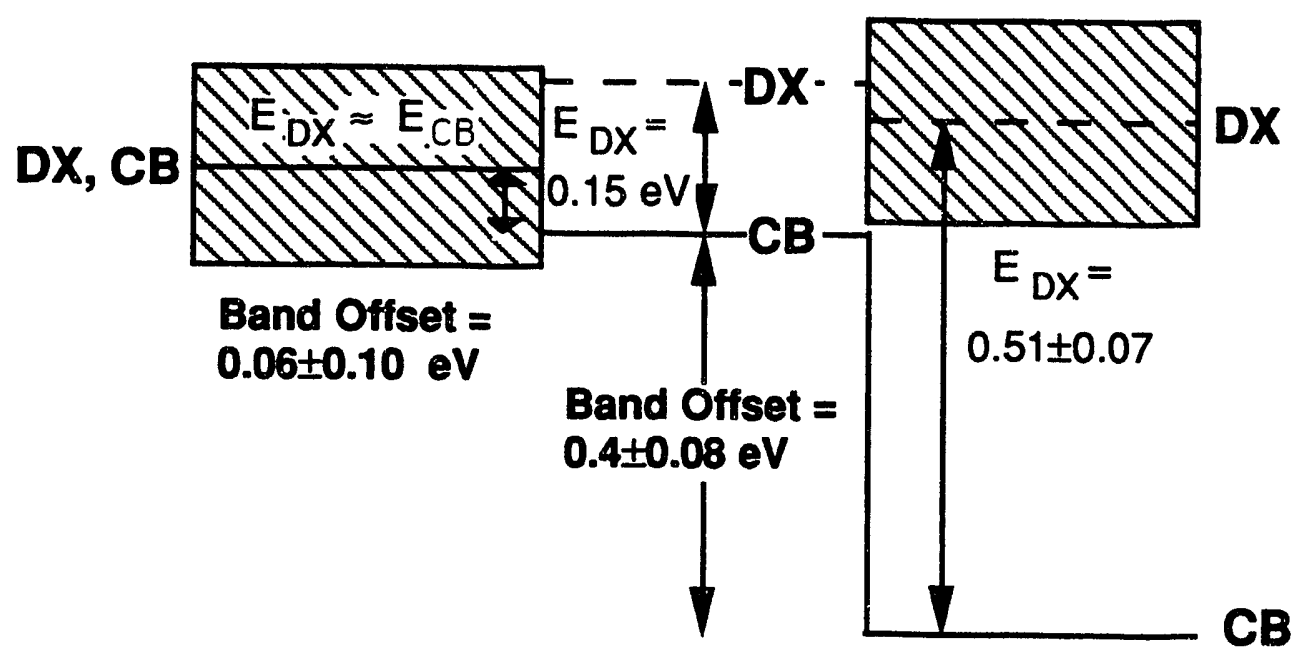

Fig. 5.4. Line-up of sulfur DX levels in InP, GaAs, and GaSb. The hatched areas represent the errors in both the energy of the DX level and the band offsets. DX levels are shown as dashed lines. 
experimental error. We also show in Figure 5.4 that a DX-like level observed in GaSt.3 also appears to line up with these other levels. It is observed in heavily doped samples (Poole; 1990, Dmowski, 1979) and at pressures of a few kbar (Kosicki, 1966), meaning that the DX level is roughly $0 \pm .05 \mathrm{eV}$ above the conduction band at zero pressure. Since the conduction band offset between GaAs and GaSb is $0.06 \pm 0.20 \mathrm{eV}$ (Van de Walle, 1989), the DX levels in all three materials line-up to within the given errors. This observation has a very useful implication. It means that if the band offsets between any given III-V semiconductor and one of the three listed here are known, then it is possible to predict the energy at which the DX level will lie in that material at zero pressure. The energy derivatives with respect to pressure or alloying can then be used to predict the conditions under which the DX center would become the stable form of the donor.

The physical justification for the energy level line-up is not clearly understood at this point, as we will now discuss. The line-up is consistent with work showing that some deep levels may be used as absolute energy level references in III-V semiconductors. (Nolte,1987; Langer, 1985). However, the levels studied in these cases were largely transition metal impurities which sit in substitutional sites. A substitutional impurity may be thought of as a vacancy with its dangling bonds into which the impurity is inserted. It can be shown (Watkins, 1983) that the electronic energy levels introduced into the gap by these transition metal impurities are made up largely of wavefunctions from the dangling-bond-like ligands of the of the nearest neighbor host atoms. In other words, the level is essentially vacancy-like. It has been suggested that energy of a neutral vacancy is equivalent to the energy of a state made up equally of contributions from the conduction and valence bands (Nolte, 1988). Since the gross features of the band structure of most III-V compounds are roughly the 
same, it is sensible that vacancy like levels would be located at the same energy in different semiconductors.

However, this discussion is in conflict with the series of equations used to determine the positions of the DX levels in InP and GaAs under pressure. These equations are based on the assumption that the derivative of the DX level with pressure was the same as that of the average pressure derivative of the lowest conduction band. Chadi (1989b) justified this assumption by stating that the impurity potential is not strong enough to require the consideration of higher lying conduction (or any valence) bands. If this is indeed the case, then the arguments stated above to justify the line-up of transition metal impurities could not be applied to the case of DX levels. A possible explanation is that: 1) the DX wavefunction does require the use of states from a large portion of the band structure, and 2) the pressure derivatives of tha DX energy level calculated taking all these states into consideration would not be so different than those calculated above. However, this is only a conjecture. So, while the physical mechanism remains unclear, it is difficult to dismiss the line-up of the levels in three different III-V semiconductors as coincidence.

This same idea was suggested previously by Hasegawa (1986) based on his observation of a line-up between DX levels in AlGaAs and GaAsP. He explained the result by claiming the DX center was an anti-bonding state with a large lattice relaxation, but his reasoning is not transparent.

In the remainder of the chapter I will discuss the use of optical techniques to determine the optical ionization and capture barrier from the shallow donor state to the deep DX state. The thermal barrier from the DX state to the shallow donor state cannot be measured optically but we will discuss a method of estimating its value using our other results. 


\subsection{Determining the Optical lonization Energy. Eopt of the InP:S DX Center}

\subsubsection{Theoretical Background of Optical Ionization of Deep Levels in}

\section{Semiconductors}

Before discussing the determination of the DX center optical ionization energy, Eopt, a discussion of the optical ionization of deep levels will be undertaken that will focus on a derivation of the equation used to fit my experimental data. The starting point for any such discussion is Fermi's golden rule, which states

$$
w=\frac{(2 \pi)^{2}}{h} \sum_{i, f} \mid H_{i f} R^{2} \delta\left(E_{i f}-h v\right)
$$

where $w$ is the transition probability per unit time, $E_{i f}$ is the energy difference between the initial and final states, and the delta function insures that the sum over all initial and final states only contains contributions from transitions which conserve energy. $\left.H_{i f}=\left\langle\psi_{i} \mid H_{p e h} \psi_{t}\right\rangle\right)$ is the matrix operator of the perturbation element representing the radiation field, where $H_{\text {per }}$ is the perturbation Hamiltonian, $\psi_{i}$ is the initial state of the transition, and $\psi_{i}$ is the final state.

We now discuss the the matrix operator $H_{i f}$ in more detail. The Hamiltonian in the presence of a radiation field is

$$
H=\frac{\left(p-\left(\frac{e}{c}\right) A\right)^{2}}{2 m}+e V=\frac{p^{2}}{2 m}+e V-\frac{e}{m c} A \cdot p,
$$


where $p$ is the momentum, $A$ is the vector potential of the radiation, $V$ is the potential, $m$ is the electron mass, and terms of order $|A|^{2}$ have been omitted. The perturbation Hamiltonian is therefore (e/mc)A·p. The vector potential for a monochromatic plane wave radiation field is

$$
A=2 \hat{A_{0} \exp }\left[i\left(\frac{\omega}{c} \times x-\omega t\right)\right]
$$

where $\hat{\varepsilon}$ and $\hat{\mathbf{n}}$ are the linear polarization and propagation direction, respectively.

Therefore

$$
H_{p e r}=-\frac{e A_{0}}{m c} \exp \left[\left(\frac{\omega}{c} \hat{n} \cdot x-\omega t\right)\right](\hat{\varepsilon} \cdot p)
$$

and

$$
w=\frac{(2 \pi)^{2} e^{2}}{h(m c)^{2}}\left|A_{d}\right|^{2}|\psi| \exp \left[i\left(\frac{\omega}{c} \hat{n} \cdot x-\omega t\right)\right](\hat{\varepsilon} \cdot p)\left|\psi \psi_{i}\right|^{2} \delta\left(E_{i f}-h v\right) .
$$

For cases of absorption by an atom of infrared or visible radiation, $(\omega / c) x=2 \pi \times / \lambda \ll<1$, implying that the exponential in Eq. 5.32 can be replaced by the value one. This simplification is referred to as the electric dipole approximation. The cross section for optical absorption is

\section{(Energy/unit time) absorbed by the atom Energy flux of the radiation field.}

The energy flux of the field is simply the product of the speed of light $c$ and the field energy density $U$, which for a classical electromagnetic field is given by 


$$
C U=\frac{1 \omega^{2}}{2 \pi} \mid A d^{2}
$$

Putting all these results together gives

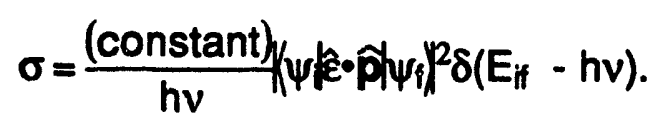

For a deep level defect, where by definition there is a strong electronphonon interaction, it is crucial to consider the lattice vibrational wavefunctions in the optical transition between different states. This is clear from Figure 5.5, which shows the vibrational wavefunctions of the initial and final states. Since transitions can only move vertically on this diagram, the transition probability is dependent on the overlap of the vibrational wavefunctions of the initial and final states.

In order to take this into account, we begin by invoking the BornOppenheimer approximation. The total defect Hamiltonian can be written

$$
H=T_{e}+T_{N}+V_{\theta e}+V_{e N}+V_{e N}
$$

where $T_{e}$ and $T_{N}$ are the kinetic energies of the electrons and ions, $V_{e e}$ and $V_{N N}$ are the electron-electron and ion-ion interactions, and $\mathrm{V}_{\mathrm{eN}}$ represents the electron-phonon interaction. The Born-Oppenheimer approximation simplifies this Hamiltonian by ignoring the kinetic energy of the ions since their mass is so much larger than that of the electrons. This effectively means that the electronic equation is solved for a fixed set of ion coordinates. The most important point is that the defect wavefunction is taken to be of the form 


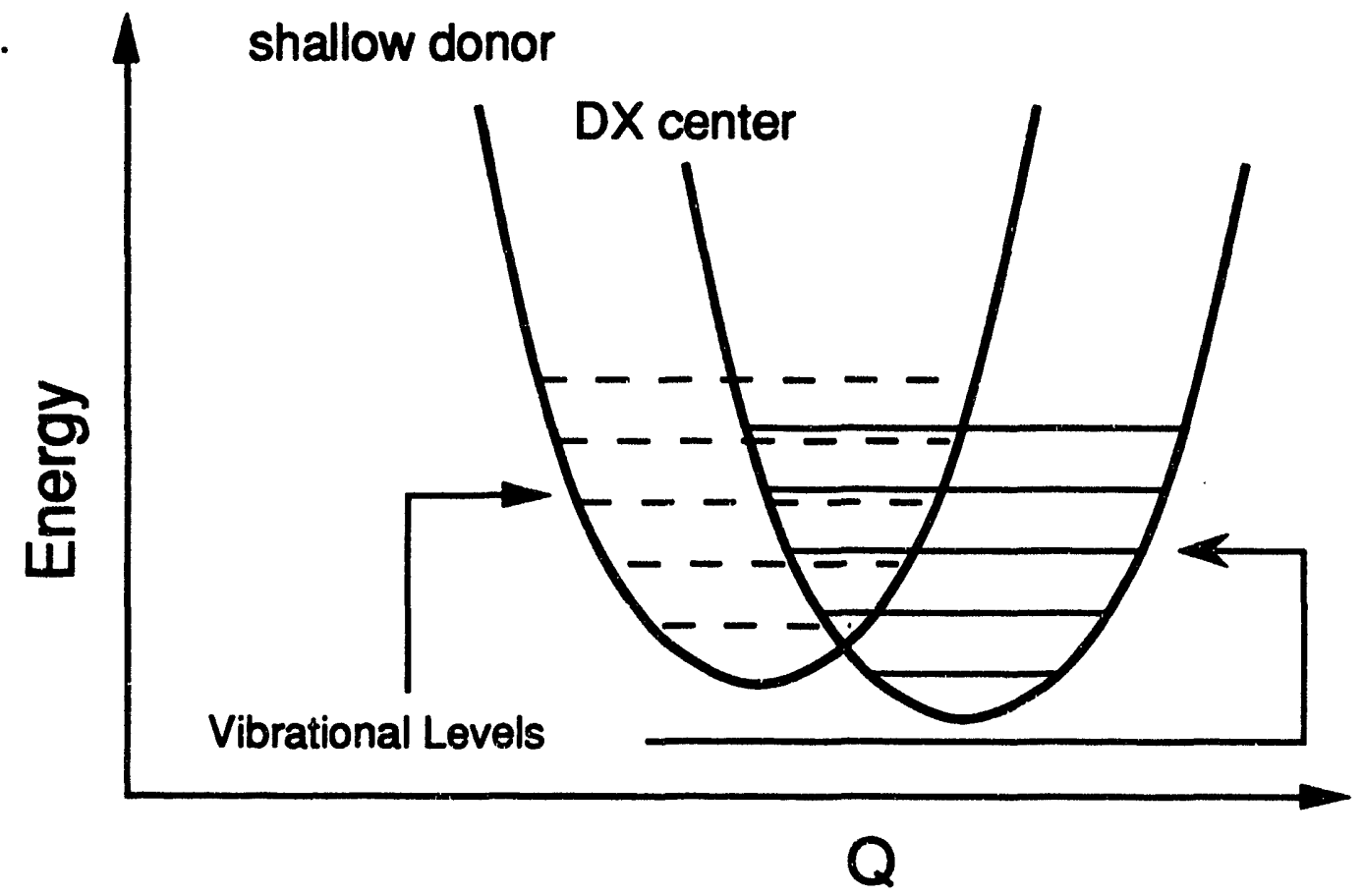

Fig. 5.5. The cross section for optical absorption by DX centers is partly determined by the overlap of vibrational wavefunctions of the initial and final states. The greater the overlap, the greater the trasition probability. Transitions can only move vertically on the diagram. 


$$
\Psi(r, Q)=\Psi(r, Q) \eta(Q)
$$

where $\Psi$ is the electronic wavefunction, $\eta$ is the vibrational wavefunction of the lattice, $r$ refers to the electronic coordinates, and $Q$ refers to the lattice coordinates. The quantity $Q$ is treated as a parameter in the equation for the electronic part of the wavefunction. The matrix element in Eq. 5.34 can then be written as

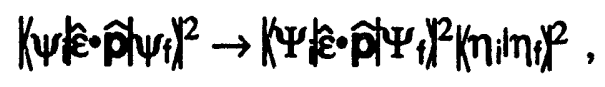

and the equation for the cross section absorption from vibrational level $m$ to vibrational level $\mathrm{m}$ ' becomes

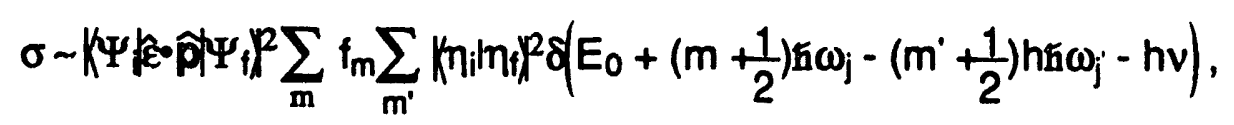

where $f m$ is the occupation number of the $m$ th level, $E_{0}$ is the energy of the zero phonon transition, and $\omega_{j}$ is the phonon frequency in the excited (primed) or ground (unprimed) state. The important result is that Eq. 5.36 has been used to separate Eq. 5.34 into separately calculable electronic and vibrational terms.

We begin by evaluating the vibrational overlap matrix element at zero temperature. This implies that the electron is initially in the $m=0$ state. We assume that the force constants defining the curvature of the two parabola are equal. The calculation is just the overlap integral between two harmonic oscillator wavefunctions, and the result is (Jaros, 1982)

$$
\left.\left|\eta_{j, 0}\right| \eta_{j, m}\right|^{2}=e^{-S} S^{m^{\prime}} / m^{\prime} !
$$


where $S=$ Huang Rhys Factor $=(1 / 2) k(\Delta Q)^{2 / \hbar \omega}$, where $\Delta Q$ is the change in the configuration coordinate between the initial and final state. $S$ is essentially the number of phonons of frequency $\omega$ emitted during the lattice relaxation, and can be taken as a measure of the strength of the electron-phonon interaction. For $S=0$, the absorption is a sharp line. For $S \neq 0$, the transition begins to broaden as more exited vibrational states become involved. For large $S$

$$
e^{-S} S^{m^{\prime}} / m^{\prime} !=\exp \left[\frac{-\left(m^{\prime}-S\right)^{2}}{2 S}\right]
$$

where $v$ is the vibrational frequency of the defect, and the line shape takes on a Gaussian appearance. Line shapes for different values of $S$ are shown in Figure 5.6. We now perform the sum over $m^{\prime}$ in Equation 5.38 keeping in mind the delta function and call the result $\mathrm{J}$.

$$
J \alpha \frac{1}{\sqrt{2 \pi S(\hbar \omega)^{2}}} \exp \left[\frac{-\left(m^{\prime} \hbar \omega-S \hbar \omega\right)^{2}}{2 S(\hbar \omega)^{2}}\right] \text {. }
$$

In order to take into account non-zero temperatures, one must form a thermal average over the initial vibrational states. This is accomplished using the standard formula for the probability for occupation $\mathrm{f}_{m}$ in Eq. 5.38 where

$$
f_{m}=\frac{\left[1-\exp \left(-\hbar \omega / k_{b} T\right)\right]}{\sum n \exp \left(-\hbar \omega / k_{b} T\right)}
$$

where $k_{b}$ is Boltzmann's constant. The result of this calculation is that (Bourgoin, 1988) 

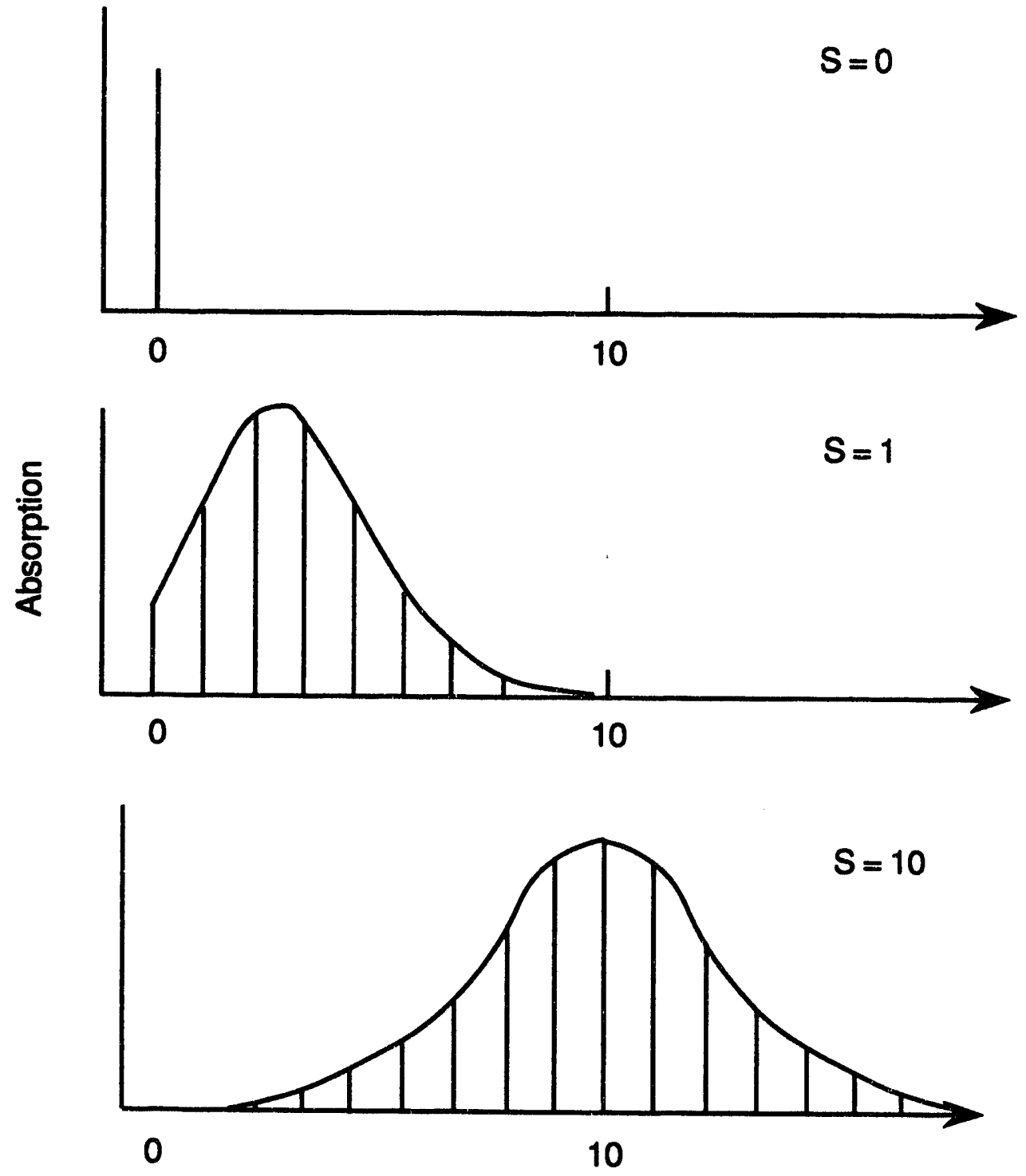

$\left(h v-E_{0}\right) / \hbar \omega$

Fig. 5.6. Shape of an absorption band as a function of the value of the Huang-Rhys parameter $\mathbf{S}$. 


$$
J=\frac{1}{\sqrt{\pi U}} \exp \left[\frac{-\left(m^{\prime} \hbar \omega-S \hbar \omega\right)^{2}}{U}\right]
$$

where $U=2 S(\hbar \omega)^{2} / \tanh \left(\hbar \omega / 2 k_{b} T\right)$.

We now use this formula to cast the equation for the optical ionization of the DX center into something close to its final form. First, we rewrite Eq. $\mathbf{5 . 3 4}$ to account for the fact that the transition occurs between the ground state and the conduction band rather than a discrete state.

$$
\sigma=\frac{(\text { constant })}{h v} \sum_{k} \mid\left(\psi \mid \hat{\varepsilon} \bullet \hat{p}_{\mid} \phi_{k}\right)^{2} \delta\left(\left|E_{i}\right|+E_{k}-h v\right),
$$

where $E_{i}$ is the impurity energy level and $E_{k}$ is the energy of the final state in the band. We then rewrite the result in Eq. 5.43 by referring to Figure 5.5 and noticing that m'hv is simply equal to $\left(h v_{\text {photon }}-\left(E_{0}+E\right)\right)$, where $E_{0}=$ is the thermal depth of the deep level with respect to the conduction band, $E$ is the energy of the final state of the transition, and $E \equiv 0$ at the conduction band minimum. It is a rather laborious calculation to find a suitable approximation for the electronic matrix element, and it does not greatly affect the line shape. We will therefore simply give the final equation for photoionization of a DX center incorporating these results. The final equation is

$$
\sigma(h v) \sim \frac{1}{h v} \int_{0}^{\infty} d E \rho(E)\left|\frac{(1-\eta) \sqrt{E}}{\mid E_{o p t}+E}+\frac{(1+\eta) \sqrt{E_{F}}}{E_{o p t}-E-\left(E_{g}+E_{A}\right) / 2}\right|^{2} U^{-1 / 2} \exp -\left(\frac{\left[h v-\left(E_{o p t}+E\right)\right]^{2}}{U}\right)
$$


where $\rho(E)$ is the density of free electron states, $E_{F}$ is the free electron Fermi energy, $E_{g}$ is the forbidden band gap , $E_{A}$ is the Penn gap, and $\eta=\exp \left(-2 E / E_{A}\right)$. This relationship was derived by Jaros (1977).

\subsubsection{Experimental Determination of the DX Center Optical lonization Energy}

In order to determine the DX center optical ionization energy experimentally, the following modification was made to the basic experimental set-up pictured in Figure 4.8. A tungsten filament lamp with the glass case broken off was placed in front of the sample. Its output was blocked by a Ge filter, making the lamp a source of infrared radiation below the band gap of Ge. The whole apparatus (equipment pictured in Fig. 4.8 plus the lamp assembly) was placed inside a Cary 2390 grating spectrometer. When the light from the grating spectrometer is of sufficient energy to optically ionize the DX centers, the sample becomes more opaque and the photocurrent through the photoconductor mounted behind the cell decreases. A black polyethylene filter was mounted in front of the photoconductor so it would not see any band edge light from the monochromator. All data was taken at $T=9 \mathrm{~K}$. A schematic of the experimental set-up is shown in Figure 5.7.

For increased sensitivity, the far infrared lamp was chopped by pulsing the voltage supply to the lamp and the photocurrent through the detector was measured using a lock-in amplifier. Because of noise problems at the frequency of the lamp pulsation, the wave used to pulse the lamp was a sine wave of frequency $\omega$ with zero DC bias. Since the power output of the lamp is independent of the direction of the current running through it, this produced a signal at $2 \omega$. Locking in on $2 \omega$ decreased noise problems tremendously. 
Monochromatic

Near Infrared

Beam

\section{Far Infrared Beam}

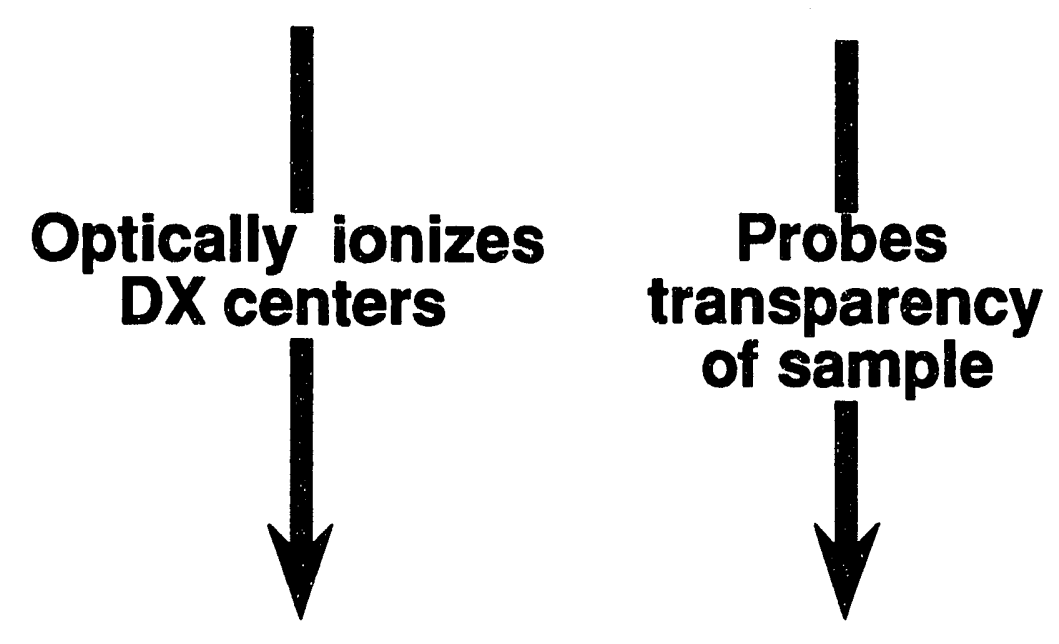

Sample

Detector

Fig 5.7. Schematic of experimental set-up used to determine the optical ionization energy and relative optical cross section of InP:S DX center 
The fraction of light going through the sample space and reaching the detector is

$$
T=I(t) /(0)=c_{0}(1-a) R^{2} \exp \left(-n \sigma_{f r e e} x\right)+a,
$$

where $I(t)=$ the photocurrent through the photoconductor as a function of time $t$, $a=$ the fraction of the sample space area not covered by the sample, $R=$ the refiection coefficient for $\operatorname{lnP}, n=$ free carrier concentration, $\sigma_{\text {free }}=$ the cross section for free carrier absorption, $x=$ the sample thickness, and $c_{0}$ is a constant taking into account all other absorption processes. We also have

$$
n=N_{D}\left(1-\exp -\left(F \sigma_{o p t} t\right)\right),
$$

where $F=$ light flux from the monochromator, $\sigma_{o p t}=$ defect optical absorption cross section, and $N_{D}=$ donor concentration. Using these expressions and making the approximation that the optical cross section for free carrier absorption is independent of the ionized impurity concentration, the following function can be fit to the photoconductor response data.

$$
\ln (I(t) / I(\infty)-a)=c_{1}-c_{2}\left(1-e^{-F} \sigma_{o p t}\right)
$$

where $c_{1}$ and $c_{2}$ are constants. Plots of the $\ln (I(t) / I(\infty)-a)$ were made as a function of time and fit with an expression of the form $\left(k_{1}+k_{2} e^{-t / \tau}\right)$. A sample fit is shown in Figure 5.8 for data using a monochromatic beam of energy $0.9 \mathrm{eV}$. This data provided a time constant, $\tau$, which is equal to the inverse of the product of the light flux and optical absorption cross section. A calibrated $\mathrm{Ge}$ photodiode was used to determine the relative values of $F$ as a function of photon energy and this allowed for the determination of the relative cross 


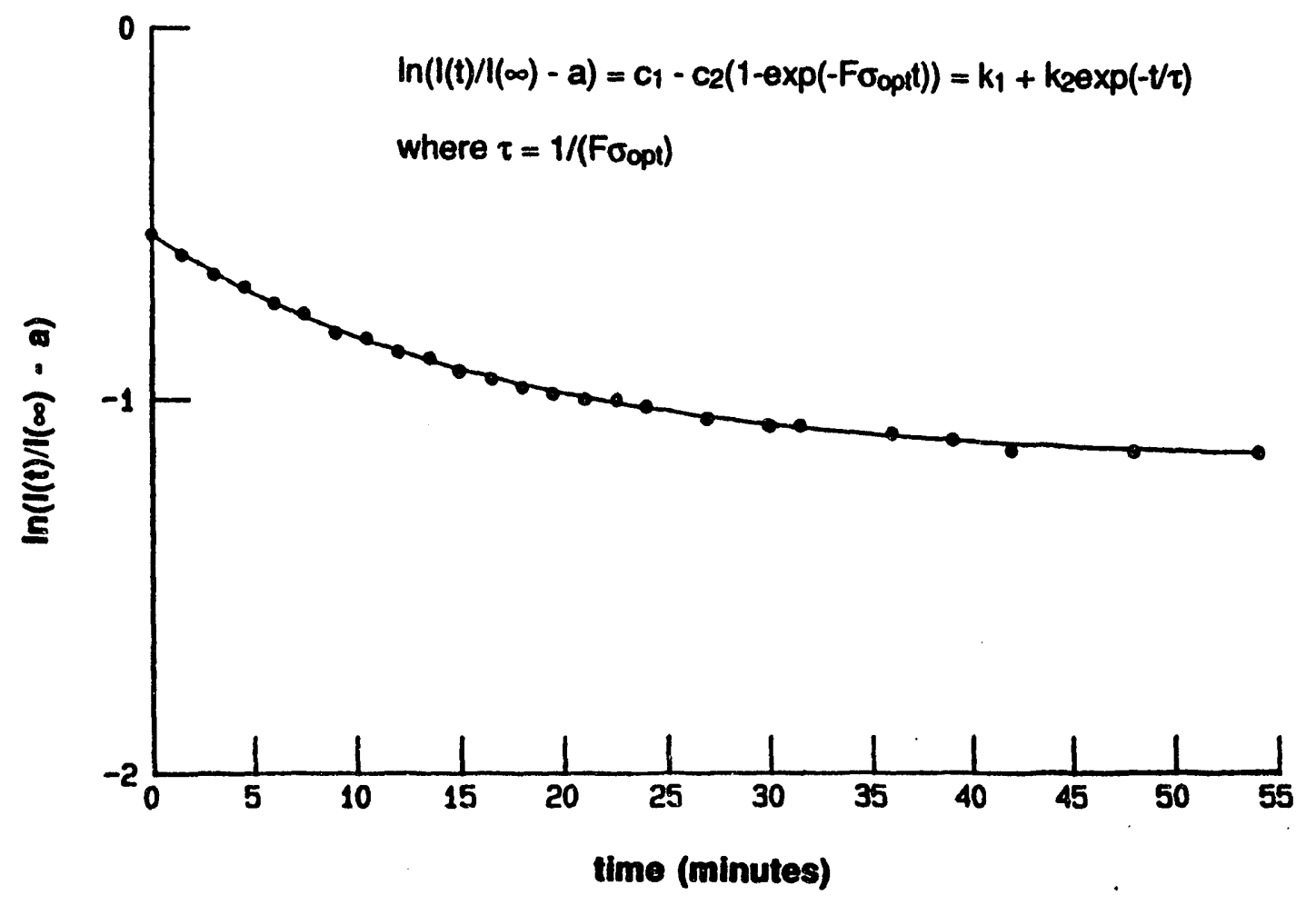

Fig. 5.8. Fit of optical ionization data using Eq. 5.48. The energy of the monochromatic beam is $0.9 \mathrm{eV}$. 
section of the S DX center as a function of energy. The cross section as a function of energy is shown in Figure 5.9 for $P=92$ kbar.

The data can be fit using the model for optical absorption by a deep level with large lattice relaxation which was used by Lang, Logan, and Jaros (1978) to fit the energy dependence of the optical ionization cross section of DX centers in $\mathrm{Al}_{x} \mathrm{Ga}_{1-\mathrm{x}} \mathrm{As}$. The following equation, whose derivation is outlined in Section 5.3.1, gives the cross section to be

$$
\sigma(h v) \sim \frac{1}{h v} \int_{0}^{\infty} d E \rho(E)\left|\frac{(1-\eta) \sqrt{E}}{E_{o p t}+E}+\frac{(1+\eta) \sqrt{E_{F}}}{E_{o p t}-E-\left(E_{g}+E_{A}\right) / 2}\right|^{2} U^{-1 / 2} \exp \left(-\frac{\left[h v-\left(E_{o p t}+E\right)\right]^{2}}{U}\right),
$$

where $\rho(E)$ is the density of free electron states, $E_{F}=11.2 \mathrm{eV}$ is the free electron Fermi energy, $E_{g}=2.2 \mathrm{eV}$ is the forbidden band gap at $T=9 \mathrm{~K}$ and $P=92 \mathrm{kbar}$,

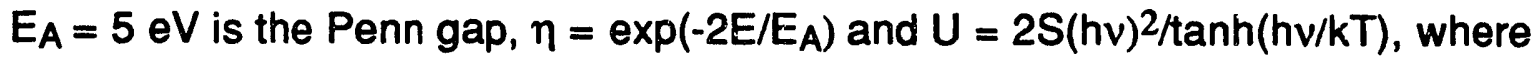
$S=$ Huang-Rhys factor and $h v=0.0085 \mathrm{eV}$ is the TA phonon energy, which is the appropriate phonon to use for DX centers. This model has two parameters, which are 1) the Frank-Condon shift of the defect $=d_{F C}=S \hbar \hbar \omega$, and 2) $E_{o p t}=E_{0}$ + Sh $\omega$. Since we do not know the temperature dependence of the cross section (we can only observe a narrow temperature range with our apparatus) we are not able to find a unique fit to our data. However, we can roughly estimate $E_{0}$, and this allows us to narrow the acceptable range for Eopt. Increasing the pressure from 82 to 92 kbar moves the DX level down in energy roughly 60 meV relative to the $\Gamma$ conduction band minimum. This implies by our previous arguments that $E_{0}$ should be in the range $60-85 \mathrm{meV}$. Using these constraints, we find good fits for our data for $E_{\text {opt }}$ between 0.86 and $1.02 \mathrm{eV}$. In Figure 5.9 we show the experimental data fit with $E_{o p t}=d_{F C}+E_{0}=0.87+0.07=0.94 \mathrm{eV}$. 


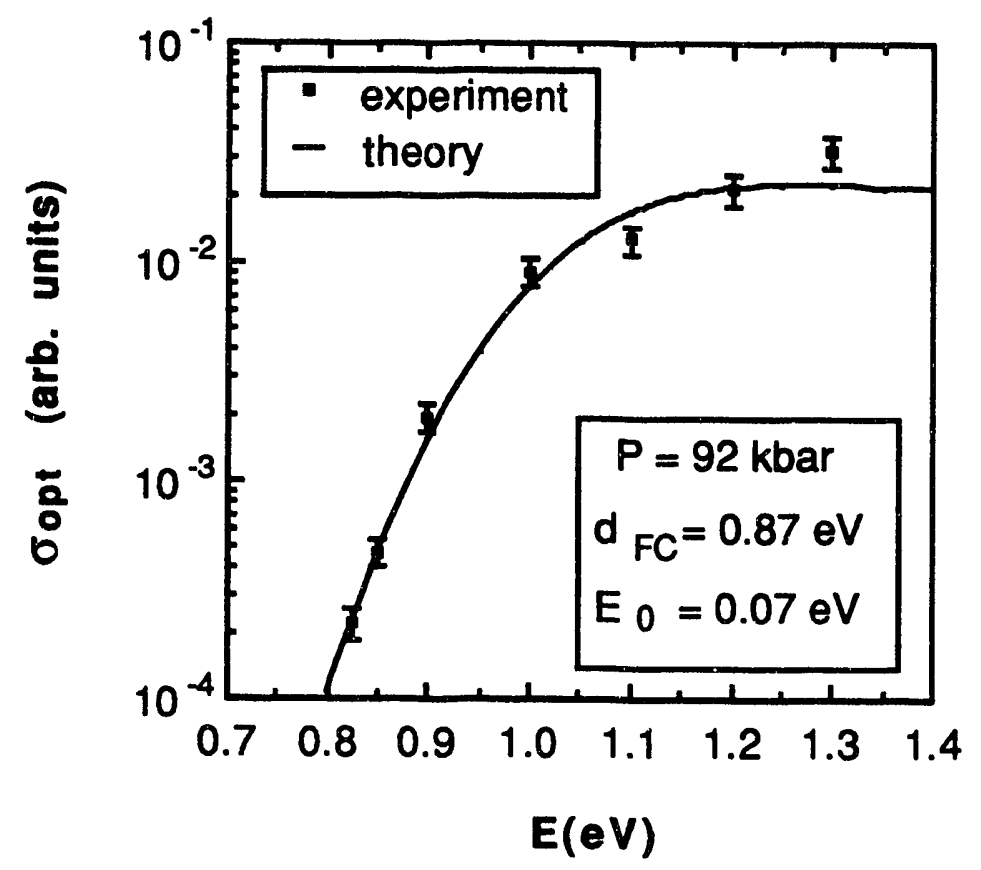

Fig. 5.9. Comparison of experimental data with theory for energy dependence of the DX center optical cross section at $P=92$ kbar. Parameters used in fit are $d_{F C}=0.87 \mathrm{eV}$ and $E_{0}=0.07 \mathrm{eV}$. 


\subsection{Determining the Thermal Capture Barrier from the Shallow State into the DX State}

\subsubsection{Modelling the Capture of Free Carriers by DX Centers}

Before discussing the experimental determination of the capture barrier, $E_{B}$, we provide a derivation of the equation used to fit the experimental data to be described in the next section. There are several possible processes by which a electron may be trapped by a defect. They differ in the way that the electron gives up its energy and are referred to as either radiative or nonradiative. Radiative capture is simply photoluminescence, i.e., the electron releases its energy by emitting a photon. There are three different non-radiative mechanisms: Auger, cascade, and multiphonon capture. In Auger capture, the electron imparts its energy to another carrier. This mechanism is thought to make a negligible contribution to the capture rate at deep levels. Cascade capture occurs when an electron is trapped by the Coulomb potential of the impurity and moves through its highly excited states by emitting and absorbing photons. Multi-phonon capture occurs due to the existence of phonon modes with strong electron-phonon coupling. The capture is accompanied by a relaxation of the lattice, and the energy from this process is dissipated by the emission of phonons. It is this process which is appropriate for describing electron capture from the shallow donor state into the deep DX level.

We consider the capture of an electron from the conduction band minimum into the DX level following the derivation of Theis (1990). For simplicity, we begin by discussing the case of a neutral DX center as pictured in 
Figure 5.10a. The total donor concentration is then $N=n_{D}^{+}+n_{D X}$, since if the center is not ionized it will be in the DX state. The concentration of these defects evolves in time according to

$$
\frac{d n_{D}^{t}}{d t}=e_{n} n_{D X}-c_{n} n_{D}
$$

where $e_{n}$ is the emission rate from the DX level into the conduction band and $c_{n}$ is the capture rate for the ionized donors. Ignoring acceptors and other donors, the free electron concentration is $n=n_{D}$. This allows Eq. 5.50 to be rewritten as

$$
\frac{d n}{d t}=e_{n}\left(N_{d}-n\right)-c_{n} n
$$

The electron capture cross section is given by

$$
c_{n}=\sigma<v>n
$$

where $v=$ the average thermal velocity of the free electrons and $\sigma$ is the elextron capture cross section, which is given by multiphonon emission theory to be

$$
\sigma=\sigma_{\infty} \exp \left(E-E_{c}\right) / k T
$$

This result can be derived in a manner very similar to the equation for optical absorption derived in Section 5.3.1. The starting equation is exactly analogous to Eq. 5.15, except that in this case $h v=0$ and the perturbation Hamiltonian is different. It turns out that $\sigma_{\infty}$ is dependent on $T^{-1 / 2}$, but this is neglected in 


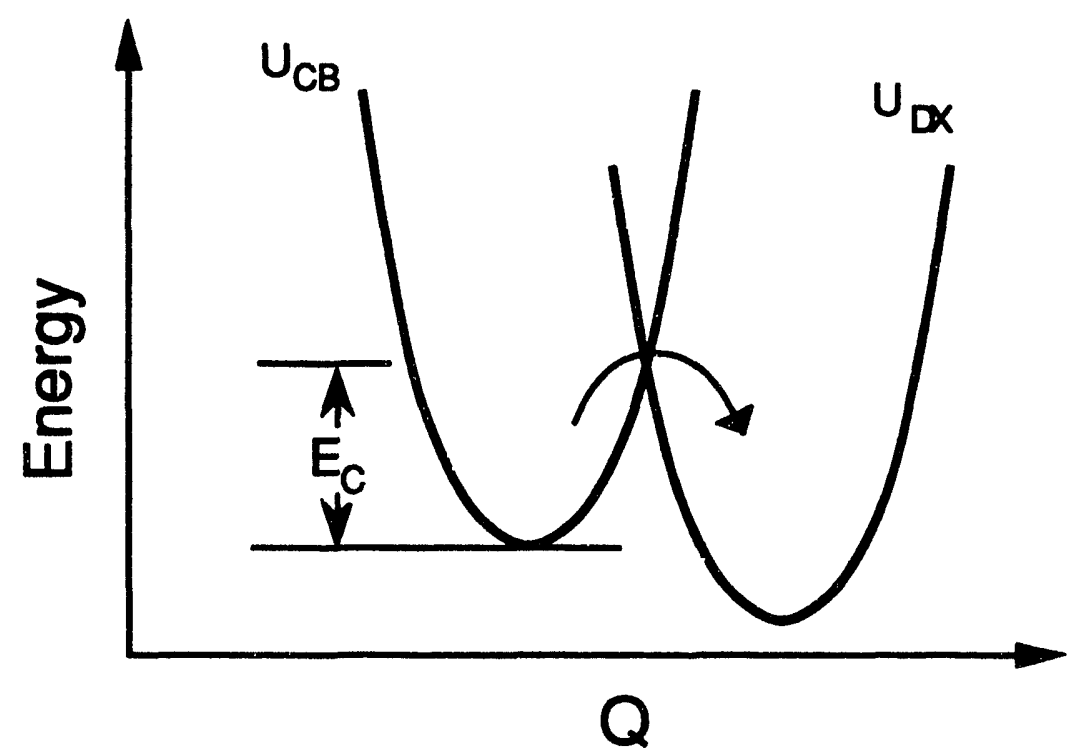

(a)

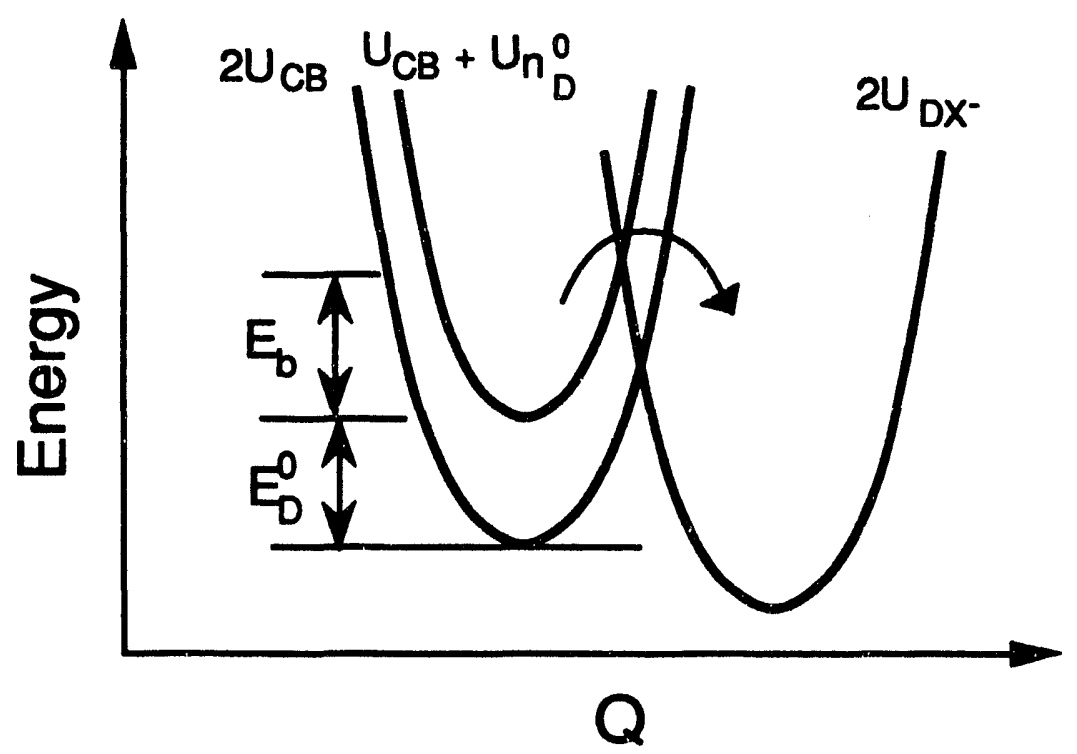

(b)

Fig. 5.10. Capture process from the shallow donor state into the deep DX state for a) a neutral DX center with no intermediate state b) a negatively charged DX center with an intermediate state. $U$ is the energy of the particular state noted by the subscript and $C B$ is conduction band (Theis, 1990). 
comparison to the exponential dependence. Substituting Eq. 5.52 into Eq. 5.51 gives

$$
d n / d t=-\sigma<v>n^{2} .
$$

Although this equation has been used to model capture data for DX centers taken with deep level transient spectroscopy, some additional assumptions were required to obtain reasonable fits. Essentially, some mechanism had to be assumed in order to obtain broader transients than predicted by Eq. 5.54, and the normal assumption was a Gaussian distribution of capture barriers. However, it was found by Mooney (1987) that data for AIGaAs samples with the same alloy content but different doping concentrations could not be fit using the same broadening parameters or activation energies. This led Theis and Mooney to develop a model for carrier capture via an excited state. The existence of such a state has been postulated in order to explain many other experiments as well (Theis, 1990; Dobaczewski, 1991; Seguy, 1990). Capture from the conduction band to a negatively charged DX center through an intermediate state is pictured in Figure 5.10b. The kinetic equation taking the excited state into account was used to model the data observed in this thesis and we now go through the derivation of this relationship, beginning by assuming a neutral DX center for simplicity.

If there is an intermediate state $n_{B}$, then $N_{D}=n_{D}+n_{D}+n_{D X}$,

$$
\frac{d n_{D}^{t}}{d t}=e_{n 1} n 8-c_{n 1} n_{D}
$$

and 


$$
\frac{d n_{D x}}{d t}=c_{n 2} n_{8}-e_{n 2} n_{D X}
$$

where $e_{n 1}$ and $e_{n 2}$ are the emission rates for transitions from the excited and DX states and $c_{n 1}$ and $c_{n 2}$ are the rates for capture into the ionized and excited states, respectively. If we assume that the transition from the excited state to the DX state is quasi-immediate, then the population of the excited state can be ignored and the emission term in Eq. 5.55 and the capture term in Eq. 5.56 can be eliminated. If the transition rate to the excited state is slow enough that the population of conduction band states is not significantly perturbed, then $c_{n 1}$ should be proportional to the electron population at the energy of the transition state, or

$$
c_{n 1}=K \frac{1}{1+\exp \left[\left(E_{B}-E_{F}\right) / k T\right]}
$$

where $K$ is a proportionality constant, $E B$ is the energy of the excited state relative to the conduction band, and $E_{F}$ is the quasi-equilibrium Fermi energy. Again neglecting the population of the excited state, $n=n_{D}$. Combining this with equations 5.55 and 5.57 in the limit $E B$ > $k T$ gives

$$
\frac{d n}{d t}=-K n \exp \left[-\left(E B-E_{F}\right) / k T\right]
$$

which is quite different from Eq. 5.54. Essentially, the electron concentration drops as trapping proceeds, lowering the quasi-Fermi level and subsequently increasing the activation energy. This stretches the capture transient in time. 
A very similar result is obtained for the case of a negatively charged DX center. Equations 5.55 and 5.56 remain the same except that $n_{D X}=n_{D X}$. It is assumed that the one electron intermediate state equilibrates rapidly with the conduction band and relatively slowly with the DX state. Therefore

$$
n_{B}=K \frac{N_{D}-n_{D X}}{1+\exp \left[\left(E_{B}-E_{F}\right) / K T\right]}
$$

Substitution of Eq. 5.59 into equation 5.56, ignoring emission, gives

$$
\frac{d n_{\bar{D} x}}{d t}=c_{n 2} \frac{N_{D}-n_{\dot{D} X}}{1+\exp \left[\left(E \dot{D}-E_{F}\right) / k T\right]}
$$

where $c_{n 2}$ is the rate constant for the capture of a second electron from the conduction band to the $\mathrm{DX}$ level. We again define $c_{n 2}=\sigma<v>n$. From the charge balance equation $n=N_{D}-n_{D}-2 n_{D X}$ we obtain the relationship $\frac{d n}{d t}=-\frac{d n B}{d t}-2 \frac{d n_{D x}}{d t}$. By combining this result with Eqs. 5.59 and 5.60, we obtain an expression for $\mathrm{dn} / \mathrm{dt}$. In the approximation $E \mathrm{~B} \gg \mathrm{kT}$, the final result is

$$
\frac{d n}{d t}=-\sigma<v>n\left(N_{D}+n\right) \exp \left[-\left(E B-E_{F}\right) / k T\right] .
$$

Using Eq. 5.53, this can be rewritten as

$$
d n / d t=-\sigma_{\infty}<v>n\left(N_{D}+n\right) \exp \left[-\left(E_{C}-E_{F}\right) / k T\right] \text {, }
$$

where $E_{c}=E_{B}+E_{b}$. 
It is important to stress the main difference between the analysis with and without an intermediate state. With an intermediate state, the capture rate depends on the quasi-Fermi level. This dependence is a result of the statistics of the population of the intermediate state. This dependence could not arise in the absence of an intermediate state for the following reason. The kinetic energy of the electrons is not available to overcome the capture barrier since the electron would have to transfer all of its energy simultaneously to phonon excitations of the defect. The large number of phonons involved makes this process extremely improbable.

\subsubsection{Experimental Determination of the Capture Barrier from the Shallow State into the DX state}

The technique used to find the thermal capture barrier from the shallow donor state into the DX state is essentially the same as that used to find the optical ionization energy. The only difference in the experimental set-up is that a lamp capable of shining white light is now placed in front of the mirror and the entire apparatus is mounted in a Digilab 80-V Fourier transform spectrometer. This allows us to use the globar of the spectrometer as our source of far infratec radiation. The sample is first cooled to $11 \mathrm{~K}$ and the donors are pumped into the metastable shallow state by shining white light on the sample. The white light lamp is then turned off and the photoconductor signal at zero path in the interferogram is recorded. The signal at zero path is the sum of all the wavelengths reaching the detector, so this is exactly analogous to the chopped photoconductor signal discussed in the section on determining the optical ionization energy. The sample is then brought up to the annealing temperature for a predetermined time, recooled, and the photoconductor signal again 
recorded. As the annealing progresses, more shallow donors transform into DX centers and the photoconductor signal increases as the sample becomes more transparent. A schematic of the experimental set-up is shown in Figure 5.11. Starting from a similar expression for $T$ as for the analysis of our optical ionization data, with the only difference being that $T=I(t) / I(\infty)$, the response of the photoconductor can be related to $\mathrm{n}$, the concentration of free carriers in the sample, by

$$
n(t)=-\left(1 / \sigma_{\text {free }} x\right)\left[\ln (1(t) / 1(\infty)-a)+c_{3}\right]
$$

where $c_{3}=$ constant. The parameters $c_{3}$ and $1 / \sigma_{\text {free }} \times$ can be fitted using $n(0)=2.1 \times 10^{18} \mathrm{~cm}^{-3}$ and $\mathrm{n}(\infty)<<(0)$. We theoretically modelled our data using the expression given by Theis et al (1990) for $n(t)$ for a negatively charged DX center which was derived in the previous section:

$$
d n / d t=-\sigma_{\infty}<v>n\left(N_{D}+n\right) \exp \left[-\left(E_{C}-E_{F}\right) / k T\right]
$$

where $\sigma_{\infty}=$ electron capture cross section at $T=\infty$ and $\langle v\rangle=$ average electron velocity. This model has $E_{c}$ as an adjustable parameter.

In order to solve this equation it was necessary to know how $E_{F}$ varied as a function of $n$. It is possible to determine this relationship by inverting the well known relation

$$
n=N_{c} F_{1 / 2}(\eta)
$$

where $\eta=E_{F} / k T, N_{C}$ is the density of states in the conduction band, and $F_{1 / 2}$ is the Fermi integral 
White Light
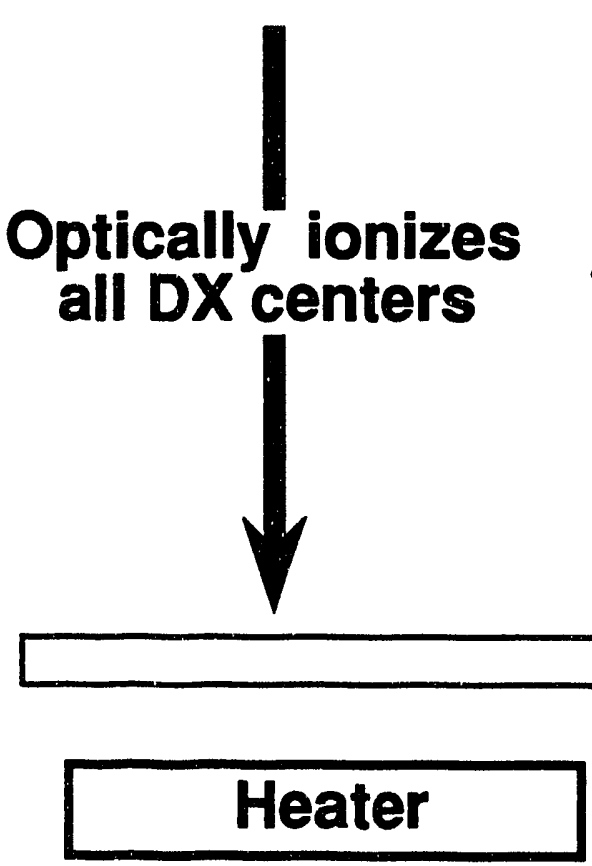

Far Infrared

Beam

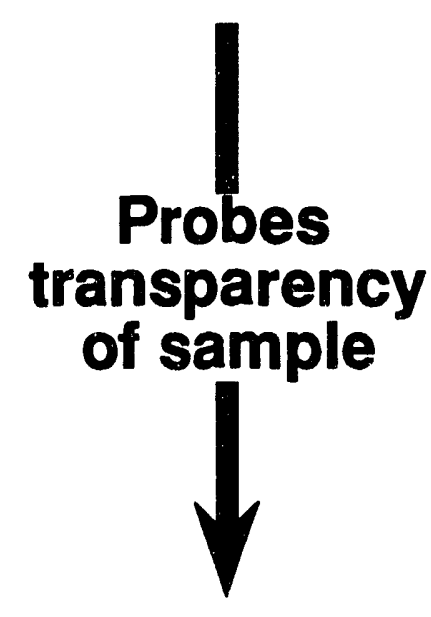

Sample

Detector

Fig 5.11. Schematic of experimental set-up used to determine the thermal capture barrier of InP:S DX center. 


$$
F_{1 / 2}(\eta)=\int_{0}^{\infty} d \varepsilon \frac{\varepsilon^{1 / 2}}{1+\exp (\varepsilon-\eta)}
$$

Because it is not possible to invert this relationship between $n$ and $\eta$ analytically, we make the approximation that (Blakemore, 1987)

$$
F_{1 / 2}(\eta)=(2 / 3) \eta^{3 / 2}\left[1+\left(\pi^{2} / 8 \eta^{2}\right)\right]
$$

which is valid over the range of concentrations observed in this study. This was combined with Eq. 5.64 and $\eta$ was found for many values of $n$. A polynomial fit was then made from the plot of $\eta$ vs. $n$ and the result was put into equation 5.61 for comparison with the data.

We performed isothermal anneals at $59 \mathrm{~K}, 63 \mathrm{~K}$ and $70 \mathrm{~K}$, and show our experimental data along with the theoretical fits for $E_{C}=0.275 \mathrm{eV}$ in Figure 5.12. Reasonable fits to our data can be obtained with $\mathrm{E}_{\mathrm{c}}$ in the range $0.23-0.33 \mathrm{eV}$.

In conclusion, we have discovered a pressure induced deep donor level in InP which has all the properties of a DX center. The pressure at which the new defect becomes more stable than the shallow donor is 82 kbar. At a pressure of 92 kbar, the optical ionization energy of this defect lies between 0.86 and $1.02 \mathrm{eV}$ and the thermal ionization energy is in the range 0.23-0.33 eV. In making the fit of the optical ionization data we estimated that $E_{0}$, the thermal depth of the $\mathrm{DX}$ center relative to the shallow donor state at 92 kbar was 60 to $85 \mathrm{meV}$. Since $E_{e}$, the emission energy from the $\mathrm{DX}$ state to the shallow donor state, is equal to $E_{c}+E_{0}$, this implies that $E_{e}$ is in the range 0.29-0.42 eV. The configuration coordinate diagram is shown in Figure 5.13 for the InP:S DX center at 92 kbar. The fact that DX centers can be formed in InP by applying pressure suggests that the existence of DX states should be very common in 


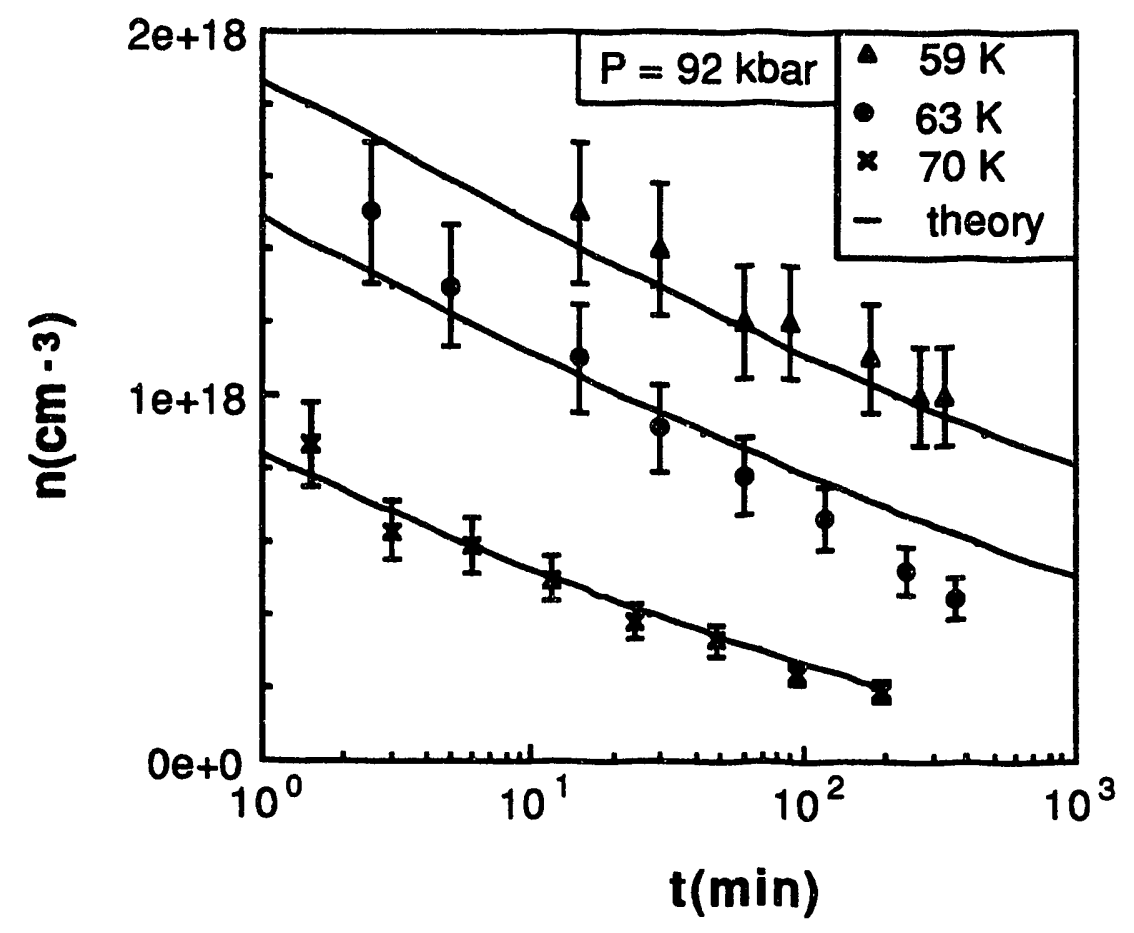

Fig. 5.12. Comparison of experimental data with theory for capture barrier from shallow donor state to $D X$ state at $P=92 \mathrm{kbar}$. Theoretical fit is for $E_{c}=0.275$ eV. 


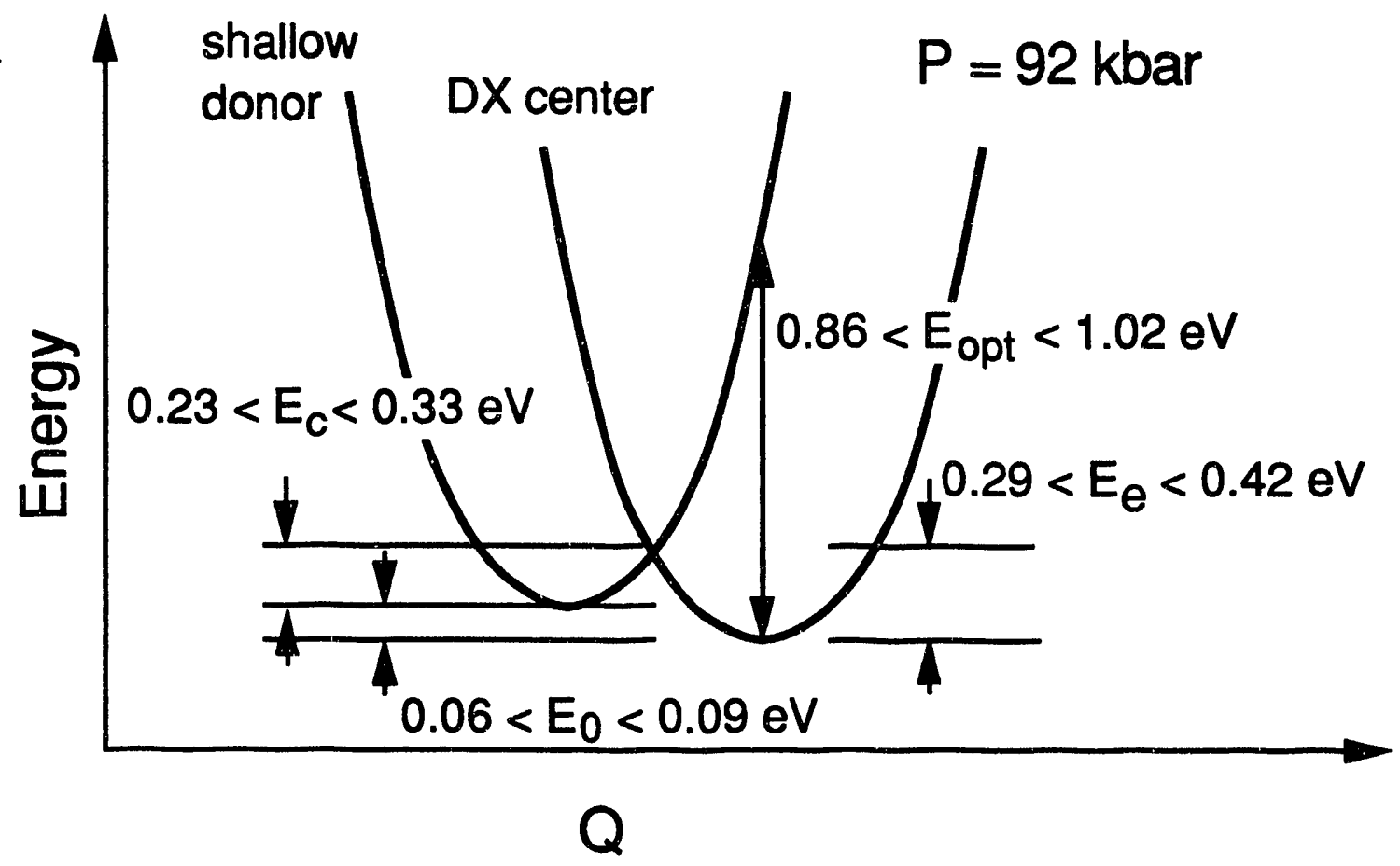

Fig. 5.13.Partially completed configuration coordinate diagram for InP:S DX centers at $P=92$ kbar. 
n-type III-V semiconductors (Khachaturyan, 1989b), though these levels may not be able to be brought into the forbidden gap. The line-up of DX levels implies that it is possible to determine the conditions under which DX centers will form in any III-V semiconductor if its band edge offsets and energy derivatives are with respect to pressure and alloying are known. 


\section{Chapter 6. Conclusion}

Determining the precise nature of the DX center has proven to be extremely challenging, and nearly every semiconductor characterization technique currently known has been used in accomplishing this task. Two questions which many experimentalists have attempted to answer are 1) What is the charge state of the DX center, and 2) What is the microscopic structure of this defect? One tool which has to proven to be invaluable in the study of DX centers is the use of hydrostatic pressure. The main effect of pressure is to modify the structure of the conduction band. In direct band gap semiconductors this has the effect of bringing deep levels down in energy with respect to the conduction band minimum. It is therefore possible in some case to bring "deep" levels which are resonant in the conduction band at ambient pressure into the forbidden gap, making them directly observable. In two semiconductors, namely GaAs and InP, the DX level can be brought into the forbidden gap in this manner.

High pressure methods were used to discover a local vibrational mode (LVM) of the DX center in GaAs:Si. In order to obtain this result, the technique of performing Fourier transform spectroscopy in a diamond anvil cell was extended to the study of semiconductor defects for the first time. A monolithic assembly containing light concentrating optics, a diamond anvil cell, and a photoconductor was constructed which provided signal-to-noise ratios far superior to previous high pressure spectroscopic results. This apparatus was used to identified a new local vibrational mode in GaAs:Si under hydrostatic pressure which was assigned the Si DX. The pressure dependence of this new mode was determined along with that of the Si substitutional donor. 
Additionally, spectroscopic data were combined with zero pressure electrical characterization to infer that the DX center was negatively charged.

Since this experiment was performed, three calculations have been undertaken to test the observed results against various theoretical microscopic models for the DX center. The calculations of Saito et al (1992) and Zhang et al (1992) show good agreement with the observed LVM frequency and frequency pressure dependence, and support the broken-bond model for the DX center proposed by Chadi and Chang. The calculations of Jones et al (1992) show that the calculated vibrational frequency for the $A_{1}$ state is also in reasonable agreement with experiment, but this assignment is not consistent with all aspects of the present work. An important prediction of both the Saito et al and Jones et al calculations is that the broken-bond configuration should have a second vibrational mode at a lower frequency than the one which was observed. Unfortunately this mode was not observed, presumably because it lies within the restrahlen region. One possibility for observing this second mode would be to repeat this experiment in InP:Si. Since we have shown that DX centers will form in this compound, and the LVM frequency for $\mathrm{Si}$ is known in InP, it is possible that the lower frequency mode could be observed in this material.

High pressure optical spectroscopy was also used to make the first observation of DX centers in InP:S just mentioned above. Because of the difficulty of making electrical measurements in a diamond anvil cell, this new DX center was characterized optically. We have experimentally determined the optical ionization energy of the DX center and the capture barrier from the shallow donor state to the deep DX state. We were also able to estimate the thermal depth of the DX center relative to the shallow donor level and the thermal ionization enthalpy from the DX state to the shallow donor state. All of 
these measurements were made at a pressure of $92 \mathrm{kbar}$. The fact that $\mathrm{DX}$ centers can be formed in InP by applying pressure suggests that the existence of DX states should be very common in n-type III-V semiconductors (Khachaturyan, 1989b). It may not always be possible to bring them into the forbidden gap using pressure or alloying, though.

It was shown that the energy levels of the S DX center in three different III-V semiconductors, namely InP, GaAs, and GaSb, lie at the same energy within our experimental error. This means that if the band offsets between any given III-V semiconductor and one of the three listed here are known, then it is possible to predict the energy at which the DX level will lie in that material at zero pressure. Furthermore, if the derivatives of the energies of the conduction band minima with respect to pressure or alloying are known, one can predict the conditions under which DX centers will be the most stable form of the donor in any III-V semiconductor. The physical justification for the line-up of these levels is not fully understood.

Although the attainment of large hydrostatic pressure creates certain experimental difficulties, its use is invaluable in the study of DX centers. The application of hydrostatic pressure allows DX centers to be observed in unalloyed materials, which makes the use of some techniques, such as local vibrational mode spectroscopy, possible. It was also necessary to apply large hydrostatic pressure in order to form DX centers in InP. DX centers now appear to be relatively well understood, and it is hoped that the study of this new InP DX center can help resolve any remaining questions. 


\section{References}

Alt, H. Ch., Appl. Phys. Lett., 55, 2736 (1989).

Anderson, P. W., Phys. Rev. Lett., 34, 953 (1975).

Baraff, G. A., Semicond. Sci. Technol., 6, B16 (1991).

Barker, A. S., Jr. and A. J. Sievers, Rev. Mod. Phys., 47, S1 (1975).

Barnett, J. D., S. Block, and G. J. Piermiani, Rev.Sci. Inst., 44, 1 (1973).

Beall, R. H., R. Murray, R. C. Newman, J. E. Whitehouse, J. Phys. C. 18, 1763 (1985).

Bell, R. J., Introductony Fourier Transform Spectroscopy, (Academic Press, New York, 1972).

Blakemore, J. S., Semiconductor Statistics, (Dover Publications, Inc., New York, 1987)

Bourgoin, J. C., and A. Mauger, Appl. Phys. Lett., 53, 749 (1988).

Bourgoin, J. C., and M. Lannoo, Point Defects in Semiconductors, (SpringerVerlag, Berlin, 1981).

Cargill, G. S., III, A. Segmüller, T. F. Kuech, and T. N. Theis, submitted to Phys. Rev. B, (1992).

Chadi, D. J., and K. J. Chang, Phys. Rev. Lett., 61, 873 (1988).

Chadi, D. J., K. J. Chang, and W. Walukiewicz, Phys. Rev. Lett., 62, 1923 (1989a).

Chadi, D. J., and K. J. Chang, Phys. Rev. B, 39, 10063 (1989b).

Chadi, D. J., private communication (1992a).

Chadi, D. J., submitted to Phys. Rev. B (1992b).

Chand, N., T. Henderson, J. Klem, W. T. Masselink, R. Fischer, Y. C. Chang, and H. Morkoc, Phys. Rev. B, Z, 692 (1973).

Chang, K. J., S. Froyen, and M. L. Cohen, Sol. St. Comm., 50, 105 (1984), and references therein.

Cheong,B. H., and K. J. Chang, to be published in Phys. Rev. B, 15, (1992). 
Colthup, N. B., L. H. Daly, and S. E. Wiberly, in Introduction to Infrared and Raman Spectrosocpy, (Academic Press, New York, 1990).

Condon, E. U., and P. M. Morse, in Quantum Mechanics, (McGraw Hill, New York, 1929).

Craford, M. G., G. E. Stillman, J. A. Rossi, and N. Holonyak, Jr., Phys. Rev., 168, 867 (1968).

Dobaczewski, L., P. Kaczor, J. M. Langer, A. R. Peaker, and I. Poole, in Proc. 20th Int. Conf. Phys. Semicon., Thessaloniki, Greece, 1990, edited by E. M. Anastassakis and J. D. Joannopoulos, (World Scientific, Singapore, 1990), p. 497.

Dobaczewski, L., and P. Kaczor, Phys. Rev. Lett., 66, 68 (1991).

Dobaczewski, L., P. Kaczor, M. Missous, A. R. Peaker, and P. R. Zytkiewicz, Phys. Rev. Lett., 68, 2508, (1992).

Dabrowski, J., R. Strehlow and M. Scheffler, Proc. 20th Int. Conf. Phys. Semicon. Thessaloniki, Greece, 1990, edited by E. M. Anastassakis and J. D. Joannopoulos, (World Scientific, Singapore, 1990), p. 497.

Dmochowski, J. E., P. D. Wang, and R.A. Stradling, Semicond. Sci. Technol., $\underline{6}$, 118 (1991).

Dmowski, L., M. Baj, M. Kubalski, R. Piotrzkowski, and S. Porowski, Inst. Phys. Conf. Ser., 43, 417 (1979).

Fellgett, J. Phys. Radium, 19, 187 (1958).

Ferraro, John R., Yibrational Soectroscopy at Hiah Pressures - The Diamond Anvil Cell, ( Academic Press, New York, 1984).

Forman, R. A.,G. J. Piermiani, J. D. Barnett, and S. Block, Science, 176, 284, (1972).

Fujisawa, T., J. Yoshino, and H. Kukimoto, Jpn. J. Appl. Phys., 29, L388 (1989).

Gibart, P., D. L. Williamson, J. Moser, P. Basmaji, Phys. Rev. Lett., 65, 1144 (1990).

Goñi, A. R., K. Syassen, K. Strössner, and M. Cardona, Phys. Rev. B, 39, 3178 (1989).

Graham, E. K., J. Geophys. Res., 91, 4630 (1936).

Haegel, N. M., E. E. Haller, and P. N. Luke, Int. J. Infra. Millim. Wav., 4 (1983). 
Haller, E. E., and J. A. Wolk, Appl. Phys. A, 53, 26, (1991).

Hasegawa, H., and H. Ohno, Jpn. J. App. Phys., 25, L319 (1986).

Hayes, T. M., D. L. Williamson, A. Outzourhit, and P. Small, J. Elect. Mat., 18, 207 (1989).

Herzberg, G., Infrared and Raman Spectra of Polyatomic Molecules, Molecular Spectra and Molecular Structure, Vol. 2, (Van Nostrand, New York, 1945).

Heyman, J. N., Ph.D. thesis.

Hjalmarson, H. P., andT. J. Drummond, Phys. Rev. Lett., 60, 2410 (1988).

Huang, K. and A. Rhys, Proc. Roy. Soc., A204, 406 (1950).

Jacquinot, P., Rep. Prog. Phys., 23, 267 (1960).

Jahren, A. H., M. B. Kruger, and R. Jeanloz, J. Appl. Phys., Z1, 1579 (1992)

Jamieson, J. C., A. W. Lawson, and N. D. Nachtrieb, Rev. Sci. Inst., 30, 1016 (1959).

Jantsch, W., Z. Williamowski, and G. O. Ostermayer, Semic. Sci. Tech. 6, B47 (1991).

Jaros, M., Phys. Rev. B, 16, 3694 (1977).

Jaros, M., Deep Levels in Semiconductors, (Adam Hilger, Ltd., Bristol, 1982)

Jayaraman, A., Rev. Mod. Phys., 55, 65 (1983).

Jones, R., and S. Öberg, Phys. Rev. B, 44, 3407 (1992).

Katsumoto, S., N. Matsunaga, Y. Yoshida, K. Sugiyama, and S. Kobayashi, Jpn. J. Appl. Phys., 29, L1572 (1990).

Khatchaturyan, K., D. D. Awschalom, J. R. Rozen, and E. R. Weber, Phys. Rev. Lett., 63, 1311, (1989a).

Khachaturyan, K., E. R. Weber and M. Kaminska, Mat. Sci. Forum, 38-41, (Trans Tech Publications, 1989b), p.1067, suggested this possibility.

Kitano, T., and M. Mizuta, Jpn. J. Appl. Phys., 26, 1806, (1987).

Kittel, C., Introduction to Solid State Physics, (John Wiley and Sons, New York, 1976). 
Kohn, in Solid State Physics, Vol. 5, ed. by F. Seitz and D. Turnbull, (Academic Press, New York, 1957), p. 256.

Kosicki, B. B., W. Paul, A. J. Strauss, and G. W. Iseler, Phys. Rev. Lett, 17, 1175 (1966).

Landolt-Börnstein, Numerical Data and Functional Relationships in Scier.se and Technology, Vol. 22, Semiconductors, ed. by O. Madelung, (SpringerVerlag, Berlin, 1987).

Lang, D. V., and R. A. Logan, Phys. Rev. Lett. 39, 635 (1977).

Lang, D. V., R. A. Logan, and M. Jaros, Phys. Rev. B, 19, 1015 (1979)

Lang, D. V., in Deep Levels in Semiconductors, edited by S. T. Pantelides (Gordon and Breach, New York, 1986) p. 489.

Langer, J. M., and H. Heinrich, Phys. Rev. Lett., 55, 1414 (1985).

Lannoo, M., Sol. St. Phenom., 10, 209, (1989).

Leszczynski, M., G. Kowalski, M. Kaminska, T. Suski, and E. R. Weber,Semic. Sci. Tech., 6, B66 (1991).

Li, M. F., P. Y. Yu, E. R. Weber, and W. Hansen, Appl. Phys. Lett., 51, 349, (1987).

Mao, H. K., P. M. Bell, R. M. Hazen, and D. J. Steinberg, J. Appl. Phys., 49, 3276 (1978).

Maude, D. K., J. C. Portal, L. Dmowski, T. J. Foster, L. Eaves, M. Nathan, M. Heiblum, J. J. Harris, and R. B. Beall, Phys. Rev. Lett., 62, 1923 (1987).

Meyer, J. R., and F. J. Bartoli, Phys. Rev. B, 36, 5989 (1987).

Mizuta, M., M. Tachikawa, H. Kukimoto, and S. Minomura, Jpn. J. Appl. Phys., 24, L143 (1985).

Mooney, P. M., N. S. Caswell, and S. L. Wright, J. Appl. Phys., 62, 4786 (1987).

Mooney, P. M., T. N. Theis, and S. L. Wright, Appl. Phys. Lett., 53, 2546 (1988).

Mooney, P. M., J. Appl. Phys. 67, 3, R1 (1990).

Mooney, P. M., private communication, (1991).

Mosser, V., S. Contreras, R. Piotrzkowski, Ph. Lorezini, J. L. Robert, J. F. Rochette, and A. Marty, in 4th Conf. Hiah Press. Semicon. Phys., (Porto-Carras, Greece, 1990), accepted for publication. 
Nelson, R. J., Appl. Phys. Lett., 31, 351 (1977).

Nolte, D. D., W. Walukiewicz, and E. E. Haller, Phys. Rev. Lett., 59, 501 (1987).

Nolte, D. D., Deep Levels in Semiconductors: Stress and Pressure Studies, Ph. D. Thesis, Physics Dept., Univ. of Calif. at Berkeley and Lawrence Berkeley Laboratory Report No. LBL-25242 (1988).

Northrop, G. A., and P. M. Mooney, J. Elec. Mater., 20, 13, (1991).

O'Reily, E. P., Appl. Phys. Lett., 55, 1409 (1989).

Piermiani, G. J., and S. Block, Rev. Sci. Inst., 46, 973 (1975).

Poole, I., M. E. Lee, I. R. Cleverly, A. R. Peaker, and K. E. Singer, Appl. Phys. Lett., 57, 1645 (1990).

Raymond, A., J. L. Robert, and C. Bernard, J. Phys., C12, 2289 (1979).

Saito, M., A. Oshiyama, and O. Sugino, Phys. Rev. B, 45, 13745 (1992).

Seal, M., High Temperatures - High Pressures, 16, 573 (1984).

Seal, M., in Hiah Pressure Research in Mineral Physics, (ed. by M. H. Manghnani and Y. Syono, Terra Scientific Publishing Company, Tokyo, 1987), p. 35.

Seguy, P., P. Y. Yu, M. F. Li, R. Leon, and K. T. Chan, Appl. Phys. Lett.,57, 2469, (1990).

Shimomura, O., et al, in Solid State Physics Under Pressure, ( ed. by S. Minomura, D. Reidel, Dordrecht, 1985), p. 351.

Skowronski, M., S. T. Neild, and R. E. Kremer, Appl. Phys. Lett., 57, 902 (1990).

Sterer, E., M. P. Pasternak, and R. D. Taylor, Rev. Sci. Inst., 61, 1117 (1990).

Tersoff, J., Phys. Rev. B, 32, 6968 (1985).

Theis, T. N., and P. M. Mooney, in Mat. Res. Soc. Symp. Proc., 163, 729 (1990).

Theis, T. N. P. M. Mooney, and B. D. Parker, J. Elect. Mat., 20.35 (1991).

Theis, W. M., and W. G. Spitzer, J. Appl. Phys., 56, 890, (1984).

Tozer, S. W., D. J. Wolford, J. A. Bradley, D. Bour, and G.B. Stringfellow, Proc. of the 19th Int. Conf. Phys. Semicon, ed. W. Zawadzki (Institute of Physics, Polish Academy of Sciences, 1988), p.881. 
Toyozowa, Y., Sol. St. Elect., 21, 1313 (1978).

Troxell , J. R. and G. D. Watkins, Phys. Rev. B., 22, 921 (1980).

Van der Pauw, L. J., Phillips Res. Repts., 13, 1 (1958).

Van de Walle, C. G., Phys. Rev. B 39, 1871 (1989).

Van Valkenburg, A., Conference Internationale Sur-les Hautes Pressions, LeCreusot, Saone-et-Loire, France (1965).

Walukiewicz, W., J. Lagowski, L. Jastrzebski, M. Lichtensteiger, and C. H.. Gatos, J. Appl. Phys., 50, 899 (1979).

Walukiewicz, W., J. Lagowski, L. Jastrzebski, P. Rava, M. Lichtensteiger, C. H. Gatos, and H. C. Gatos, J. Appl. Phys., 51, 2659 (1980).

Walukiewicz, W., Phys. Rev. B, 41, 10218 (1990).

Watkins, G. D., and J. W. Corbett, Phys. Rev., 121, 1001 (1961).

Watkins, G. D., Physica, 177B \& 188B, 9 (1983)

Weir, C. E., E. R. Lippincott, A. Van Valkenburg, and E. N. Bunting, J. Res. Nat. Bur. Stand. Sec. A, 63. (1959).

Williams, Q., and R. Jeanloz, in Molten Salt Techniques, Vol 4, ed. R. J. Gale and D. G. Lovering, (Plenum Publishing Corporation, 1991).

Williamowski, Z., J. Kossut, W. Jantsch, and G. Ostermayer, Semic. Sci. Tech., 6, B38 (1991).

Wolk, J. A., M. B. Kruger, J. N. Heyman, W. Walukiewicz, R. Jeanloz, and E. E. Haller, Semic. Sci. Tech., 6, B78 (1991a).

Wolk, J. A., M. B. Kruger, J. N. Heyman, W. Walukiewicz, R. Jeanloz, and E. E. Haller, Phys. Rev. Lett. 66, 774 (1991b).

Wolk, J. A., W. Walukiewicz, M. L. W. Thewalt, and E. E. Haller, Phys. Rev. Lett., 68, 3619 (1992a).

Wolk, J. A., M. B. Kruger, J. N. Heyman, J. W. Beeman, J. G. Guitron, E. D. Bourret, W. Walukiewicz, R. Jeanloz, and E. E. Haller, Proc. of the 16th Int. Conf. on Defects in Semicon., in Mat. Sci. Forum, Vol. 83-87, Eds. G. D. Davies, G. G. DeLeo, and M. Stavola, (Trans Tech Publications, Switzerland, 1992b).

Woodhead, J., R. C. Newman, A. K. Tipping, J. B. Clegg, J.A. Roberts, and I. Gale, J. Phys. D: Appl. Phys., 18, 1575 (1985). 
Yamiguchi, E., K. Shiraishi, K., and T. Ohno, J. Phys. Soc. Jpn., 60, 3093 (1991).

Zhang, S. B., D. Erskine, M. L. Cohen, and P. Y. Yu, Sol. St. Comm., 71, 369 (1989) 

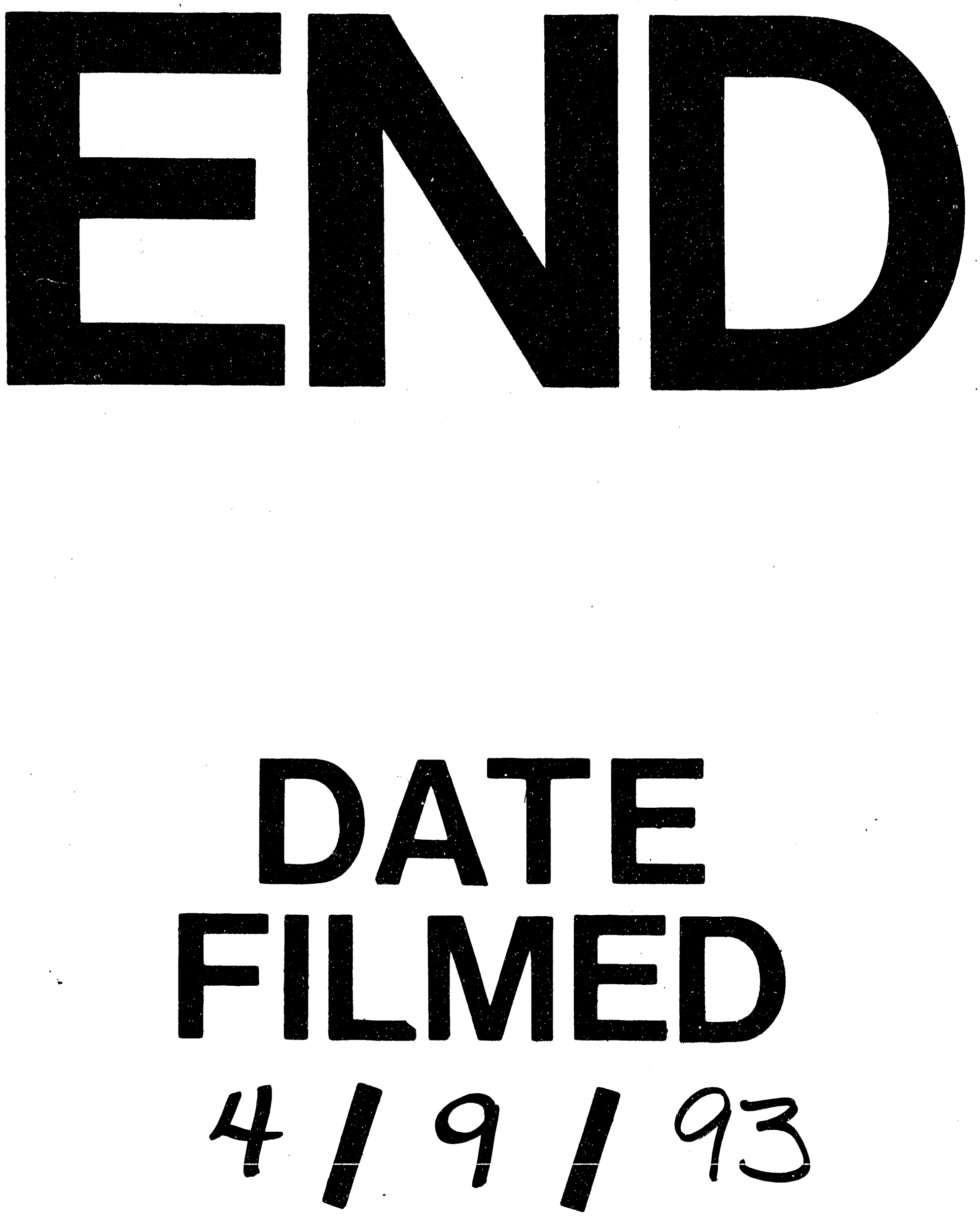
